\title{
Morphophylogenetic study of Sydowiellaceae reveals several new genera
}

\section{Senanayake IC 1,2,3, Maharachchikumbura SSN ${ }^{4}$, Jeewon $\mathbf{R}^{5}$, Promputtha I ${ }^{6}$, Al-Sadi AM ${ }^{4}$, Camporesi $\mathrm{E}^{7,8,9}$ and Hyde KD ${ }^{1,2,3}$}

${ }^{1}$ Key Laboratory for Plant Diversity and Biogeography of East Asia, Kunming Institute of Botany, Chinese Academy of Science, Kunming 650201, Yunnan, China

${ }^{2}$ East and Central Asia, World Agroforestry Centre, Kunming 650201, Yunnan, China

${ }^{3}$ Centre of Excellence for Fungal Research, Mae Fah Luang University, Chiang Rai, Thailand

${ }^{4}$ Department of Crop Sciences, College of Agricultural and Marine Sciences, Sultan Qaboos University, P.O. Box 34, Al-Khod 123, Oman

${ }^{5}$ Department of Health Sciences, Faculty of Science, University of Mauritius, Mauritius, 80837

${ }^{6}$ Department of Biology, Faculty of Science, Chiang Mai University, Chiang Mai 50200, Thailand

${ }^{7}$ A.M.B. Gruppo Micologico Forlivese, Antonio Cicognani, Via Roma 18, Forlì, Italy

${ }^{8}$ A.M.B. Circolo Micologico, Giovanni Carini, 314 Brescia, Italy

${ }^{9}$ Società per gli Studi Naturalistici della Romagna, 144 Bagnacavallo, RA, Italy

Senanayake IC, Maharachchikumbura SSN, Jeewon R, Promputtha I, Al-Sadi AM, Camporesi E, Hyde KD. 2017 - Morphophylogenetic study of Sydowiellaceae reveals several new genera. Mycosphere 8(1), 172-217, Doi 10.5943/mycosphere/8/1/15

\begin{abstract}
Sydowiellaceae is a poorly studied family of the order Diaporthales, comprising a collection of morphologically diversified taxa. Eleven genera have been previously listed under this family. In this study, we provide a DNA sequence-based phylogeny for genera of Sydowiellaceae based on analyses of a combined LSU, ITS, RPB2 and TEF sequence dataset to establish the boundaries within the family. We introduce, describe, and illustrate three new genera, Italiomyces, Ranulospora, and Tenuiappendicula, based on newly collected specimens. Another three new genera, Alborbis, Breviappendix, and Paragnomonia are introduced to accommodate Cryptodiaporthe galericulata, Gnomonia rosae, G. rostellata, G. rubi, and G. fragariae. Alborbis galericulata comb. nov., Breviappendix rubi comb. nov., $B$. rosae comb. nov., B. rostellata comb. nov. and Paragnomonia fragariae comb. nov. are proposed. Two new species, Sydowiella centaureii and Sillia karstenii, are introduced with descriptions and illustrations. Winterella is replaced with a new genus Tortilispora typified by $T$. aurantiaca. Three new combinations are proposed in Tortilispora. We synonymize Stegophora under Lambro based on morphological similarities. An updated key for genera of Sydowiellaceae is presented.
\end{abstract}

Key words - Alborbis, Breviappendix, Italiomyces, Paragnomonia, Ranulospora, Sillia karstenii, Sydowiella centaureii, Tenuiappendicula, Tortilispora.

Submitted 10 January 2017, Accepted 8 February 2017, Published 20 February 2017

Corresponding Author: Itthayakorn Promputtha - e-mail - ppam118@gmail.com 


\section{Introduction}

Sydowiellaceae Lar. N. Vassiljeva was established to accommodate the genus Sydowiella Petr., which is typified by S. fenestrans (Duby) Petr., and a collection of morphologically diverse fungal taxa whose intergeneric relationships are still uncertain (Maharachchikumbura et al. 2015). Sydowiellaceae is a phylogenetically, well-supported, monophyletic family within Diaporthales (Kruys \& Castlebury 2012), but is morphologically poorly studied. To date, Sydowiellaceae comprises eleven genera (Maharachchikumbura et al. 2015, 2016) and 43 species (Index Fungorum 2017).

The family Sydowiellaceae is generally characterized by taxa with solitary or aggregated, erumpent to immersed ascomata, with wide papilla, a peridium comprising an outer layer of brown-walled cells of textura globulosa and inner layer of hyaline cells of textura angularis, cylindrical to cylindro-clavate or broadly fusoid unitunicate asci, with a J-, sometimes bi-lobed apical ring, and hyaline or greenish-brown, 0-11-septate, ellipsoidal or filiform ascospores that sometimes have apical and basal appendages (Maharachchikumbura et al. 2016).

The family Sydowiellaceae also includes the genera Cainiella E. Müll. typified by $C$. johansonii (Rehm) E. Müll., Calosporella J. Schröt typified by C. innesii (Curr.) J. Schröt., Chapeckia M.E. Barr typified by C. nigrospora (Peck) M.E. Barr, Hapalocystis Auersw. ex Fuckel typified by $H$. berkeleyi Fuckel, Lambro Racib. typified by L. insignis Racib., Rossmania Lar. N. Vassiljeva typified by R. ukurunduense Lar. N. Vassiljeva, Stegophora Syd. \& P. Syd. typified by S. ulmea (Fr.) Syd. \& P. Syd., Sillia P. Karst. typified by $S$. ferruginea (Pers.) P. Karst., Uleoporthe Petr typified by U. orbiculata (Syd. \& P. Syd.) Petr. and Winterella (Sacc.) Kuntze typified by W. aurantiaca (Wehm.) J. Reid \& C. Booth (Maharachchikumbura et al. 2015, 2016). Due to morphological plasticity, the taxonomic affinities of most genera within Sydowiellaceae have been uncertain and they were referred to Diaporthales genera incertae sedis (Rossman et al. 2006). Despite morphological based taxonomic discrepancies, DNA sequence based phylogenies have consistently reported Sydowiellaceae to be a well-supported monophyletic family comprising the above-mentioned genera (Morocko 2006, Rossman et al. 2007, Kruys \& Castlebury 2012, Maharachchikumbura et al. 2015, 2016). Stilbospora Pers., Hendersonia E.J. Butler, and Dothiorella Sacc. have been reported as asexual morphs for Hapalocystis associated with ascomata on the natural substrate (Wehmeyer 1941, Barr 1978, Jaklitsch \& Voglmayr 2004), While Glawe (1985) and Liu et al. (2015) reported a phoma-like asexual morph for $H$. berkeleyi in culture. Cylindrosporella Höhn. was reported as the asexual morph of Stegophora by Kobayashi (1970).

Members of Sydowiellaceae occur on herbaceous plants, dicotyledonous and hardwood trees, as saprobes, endophytes or pathogens. Stegophora ulmea was reported as a pathogen on living leaves of Ulmus americana, while Sydowiella depressula was reported on leaves and stem cankers of cultivated black berry (Rubus fruticosus) (Nordskog et al. 2003). Sydowiella fenestrans has been recorded on leaves and dead stems of various plants. Cainiella johansonii was reported on both living and dead leaves and petioles of Dryas species, while Cainiella borealis was found as a saprobe on dead branches of Cassiope tetragona (Kruys \& Castlebury 2012). Hapalocystis berkeleyi forms stromatic fruiting bodies on dead woody plants. Collections of Uleoporthe orbiculata were recorded on living and fallen leaves. There are reports that $U$. orbiculata and many other taxa in this family may have biotrophic lifestyles (Cannon 2001, De Silva et al. 2016).

Species in Gnomoniaceae appear to be similar to Sydowiellaceae. Kruys \& Castlebury (2012) showed that Cryptodiaporthe galericulata, Gnomonia fragariae, G. rostellata, and G. rubi (Gnomoniaceae) clustered in Sydowiellaceae based on ITS sequence analysis. Morocko (2006) also showed a close affinity of Gnomonia rosae to Sydowiellaceae. Voglmayr \& 
Jaklitsch (2014) introduced Calosporella for Prosthecium innesii and accommodated it as Calosporella innesii.

Here we provide an updated phylogeny based on DNA sequence analyses of taxa of Sydowiellaceae to clarify intergeneric relationships. In addition, we introduce and describe three new monotypic genera based on fresh collections, another three new genera based on combinations of existing species with molecular data and two new species in existing genera based on fresh collections. Winterella is replaced with a new genus Tortilispora typified by $T$. aurantiaca. Stegophora is synonymized under Lambro based on its morphology. An updated taxonomic key of genera in Sydowiellaceae is also provided.

\section{Materials \& Methods}

\section{Specimen collection, morphological examination and photomicrography}

Collections were made in Italy from 2013 to 2016. Specimens were placed in paper bags and collection details noted. Samples were brought to the laboratory and examined under a stereomicroscope to observe the characteristics of the fruiting bodies. Macromorphological characters were photographed using a digital camera fitted to the stereomicroscope. A few fruiting bodies were transferred to a drop of water mounted on a slide using fine needles or forceps for rehydration and they were crushed to observe internal structures. Cross sections of fruit bodies were hand cut with a razor blade and mounted in a drop of water on a slide. Morphological characteristics of ascomata/conidiomata, asci, ascospores/conidia and other tissues associated with ascomata/conidiomata were photographed using a digital camera fitted to ta compound microscope. Melzer's reagent, Indian ink and $5 \% \mathrm{KOH}$ were used where necessary to stain fungal structures. All microphotographs were arranged using Adobe Photoshop CS6 (v. 13.0.1.1) and all measurements were made with Tarosoft (R) Image Frame Work (v. 0.9.0.7). Specimens were preserved and deposited at Mae Fah Luang University herbarium (MFLU) and BIOTEC Bangkok Herbarium (BBH). Facesoffungi and Index Fungorum numbers were registered (Jayasiri et al. 2015, Index Fungorum 2017). New species were resolved following recommendations as outlined by Jeewon \& Hyde (2016).

\section{Single spore isolation}

A few fruiting bodies were picked from substrates using a sterilized needle and placed in a few drops of sterilized distilled water on a sterilized cavity slide as detailed in Chomnunti et al. (2014). Fruiting bodies were crushed to separate the spores and obtain a spore suspension. The suspension was inoculated on to malt extract agar (MEA) and incubated at $18{ }^{\circ} \mathrm{C}$. Germinating ascospores were aseptically transferred to new culture plates. Colonies were photographed and characters were noted. Living cultures are deposited at Mae Fah Luang University Culture Collection (MFLUCC) and the Thailand Bioresource Research Center (TBRC).

\section{Promoting asexual morphs}

Dried and sterilized grass leaves or pine needles were laid parallel to the colony margin of pure cultures which were grown on water agar (Phookamsak et al. 2015). Conidiomata produced on both water agar and sterilized pine needles were observed after few weeks of incubation at $18{ }^{\circ} \mathrm{C}$. Morphological characteristics were examined as mentioned above.

\section{Herbarium material examination and re-drawing}

Types and other relevant authentic specimens were loaned from accessible herbaria (New York State Museum (NYS), Swedish Museum of Natural History (S), 
Naturhistorisches Museum Wien (W), and International Mycological Institute (IMI)). A small part of the herbarium specimen was cut and rehydrated using water or $5 \% \mathrm{KOH}$. Micromorphological characters were observed from rehydrated ascomata and photography was done as previously described. Some specimens were redrawn based on previously illustrated authentic specimens.

\section{DNA extraction, PCR amplification, sequencing and phylogenetic analyses}

Scraped fresh fungal mycelia grown on MEA for 4 weeks at $18{ }^{\circ} \mathrm{C}$, or internal content of ascomata or conidiomata were used for genomic DNA extraction using a modified protocol of Jeewon et al. (2002). PCR amplification and sequencing of large subunit ribosomal RNA (LSU) region using the primer pair LROR/LR5, internal transcribed spacer (ITS) regions using primer pair ITS4/ITS5, RNA polymerase II gene (RPB2) region using the primer pair fRPB2-5F/fRPB2-7cR, and translation elongation factor (TEF) region using primer pair 728F/986R were performed (Vilgalys \& Hester 1990, White et al. 1990, Carbone \& Kohn 1999, Liu et al. 1999).

Each PCR reaction contained $0.3 \mu 1$ of TaKaRa Ex-Taq DNA polymerase, $12.5 \mu 1$ of 2 $\times$ PCR buffer, $2.5 \mu 1$ of dNTPs, $1 \mu 1$ of forward and reverse primers, $1 \mu 1$ of DNA template and was adjusted with $6.5 \mu \mathrm{l}$ of double-distilled water to a total volume of $25 \mathrm{ml}$. Amplification reactions were performed in a thermal-cycler (BIORAD 1000TM Thermal Cycler, Bio-Rad Laboratories, Hercules, California). The temperature profile for both ITS and LSU was an initial denaturing step for $2 \mathrm{~min}$ at $94{ }^{\circ} \mathrm{C}$, followed by 35 amplification cycles of denaturation at $94{ }^{\circ} \mathrm{C}$ for $60 \mathrm{~s}$, annealing at $58{ }^{\circ} \mathrm{C}$ for $60 \mathrm{~s}$ and extension at $72{ }^{\circ} \mathrm{C}$ for $90 \mathrm{~s}$ and a final extension step of $72{ }^{\circ} \mathrm{C}$ for $10 \mathrm{~min}$ (Jeewon et al. 2004, Phillips et al. 2008). The temperature profile for the RPB2 was, initial denaturation at $94{ }^{\circ} \mathrm{C}$ for $2 \mathrm{~min}$, followed by 35 amplification cycles of denaturation at $95{ }^{\circ} \mathrm{C}$ for $45 \mathrm{~s}$, annealing at $57{ }^{\circ} \mathrm{C}$ for $50 \mathrm{~s}$ and extension at $72{ }^{\circ} \mathrm{C}$ for $90 \mathrm{~s}$. (Liu et al. 1999). The temperature profile for the TEF was, initial denaturation at $94{ }^{\circ} \mathrm{C}$ for $2 \mathrm{~min}$, followed by 35 amplification cycles of denaturation at $95{ }^{\circ} \mathrm{C}$ for $30 \mathrm{~s}, 58{ }^{\circ} \mathrm{C}$ for $50 \mathrm{~s}, 72{ }^{\circ} \mathrm{C} 60 \mathrm{~s}$. (Glass \& Donaldson 1995). All amplified PCR products were determined by electrophoresis at $90 \mathrm{~V} / \mathrm{cm}$ for $40 \mathrm{~min}$ in $1 \%$ agarose gel stained with ethidium bromide $(0.5 \mathrm{mg} / \mathrm{mL})$. The gel was visualized under a UV transilluminator to estimate the fragment size. PCR products were purified and sequenced with both primers at the Sunbiotech Company, Beijing, China. Sequences were edited and assembled with DNASTAR Lasergene (v.7.1) to obtain consensus sequences. The sequences generated in this study were supplemented with additional sequences obtained from GenBank (Table 1) based on blast searches and published literature. Multiple sequence alignments were generated with MAFFT v. 7 (http://mafft.cbrc.jp/alignment/server/index.html) and the alignment was manually improved with BioEdit v. 7.0.5.2 (Hall 1999).

Phylogenetic analyses of the sequence data consisted of Maximum Likelihood, Bayesian inference and maximum parsimony. Maximum likelihood (ML) analysis was performed by RAxMl GUI v.1.3 (Stamatakis et al. 2008, Silvestro \& Michalak 2012). The search strategy was set to rapid bootstrapping and the analysis was carried out using the GTRGAMMAI model of nucleotide substitution with 1000 replicates. The model of evolution was estimated by using MrModeltest 2.3 (Nylander 2004) and GTR+I+G was selected as the model for Bayesian analyses. Bayesian inference in MrBayes v. 3.2.1 (Ronquist et al. 2012) was performed with default settings, running four chains over 3 million generations and sampling each $100^{\text {th }}$ tree. The first 6000 of the 3000000 saved trees were discarded and the consensus tree was based on the remaining 2994000 trees. Maximum parsimony analysis (MP) was performed using PAUP v. $4.0 b 10$ (Swofford 2003). Ambiguously aligned regions were excluded and gaps were treated as a missing data. All characters were unordered and given equal weight. Trees were inferred using the heuristic 
Table 1. Isolates used in the analyses and their GenBank and culture accession numbers. Newly generated sequences are in bold.

\begin{tabular}{|c|c|c|c|c|c|}
\hline \multirow{2}{*}{ Taxa } & \multirow{2}{*}{$\begin{array}{c}\text { Culture accession } \\
\text { numbers }\end{array}$} & \multicolumn{4}{|c|}{ GenBank accession numbers } \\
\hline & & ITS & LSU & RPB2 & TEF \\
\hline Asterosporium asterospermum & KT2125 & - & AB553743 & - & - \\
\hline Asterosporium asterospermum & CBS 112404 & - & AB553745 & - & - \\
\hline Asterosporium asterospermum & KT2101 & - & AB553742 & - & - \\
\hline Asterosporium asterospermum & KT2138 & - & AB553744 & - & - \\
\hline Breviappendix rubi & MFLUCC 16-1205 & KY523473 & KY523491 & - & - \\
\hline Breviappendix rubi & MFLUCC 16-1205A & KY523474 & KY523492 & - & - \\
\hline Breviappendix rubi & MFLUCC 16-1205B & KY523475 & KY523493 & - & - \\
\hline Cainiella borealis & Kruys 725 (UPS) & JF701921 & - & - & - \\
\hline Cainiella johansonii & Kruys 731 (UPS) & - & JF701920 & - & - \\
\hline Calosporella innesii & AR 3639 & JF681965 & EU683071 & - & - \\
\hline Calosporella innesii & AR 3925 & JF681966 & - & - & - \\
\hline Calosporella innesii & AR 3831 & JF681964 & - & - & - \\
\hline Chapeckia nigrospora & CBS 125532 & JF681957 & EU683068 & - & - \\
\hline Cryphonectria macrospora & CBS 109764 & EU199182 & AF408340 & EU220029 & - \\
\hline Cryphonectria parasitica & ATCC 38755 & AY141856 & EU199123 & DQ862017 & EU222014 \\
\hline Cryptodiaporthe galericulata & AR 3890 & JF681967 & - & - & - \\
\hline Cryptodiaporthe galericulata & AR 4004 & JF681968 & - & - & - \\
\hline Cryptodiaporthe galericulata & AR 3811 & JF681969 & - & - & - \\
\hline Cryptodiaporthe galericulata & AR 4027 & JF681970 & - & - & - \\
\hline Cytospora hippophaes & CFCC 89640 & KF765682 & KF765698 & KF765714 & KP310865 \\
\hline Diaporthe eres & AR 5193 & KJ210529 & - & - & $\mathrm{KJ} 210550$ \\
\hline Diaporthe eres & PS57 & JF430494 & JF704176 & - & - \\
\hline Gnomonia cf rosae & BPI $877452 B$ & EU254935 & EU255157 & - & - \\
\hline Gnomonia fragariae & CBS 146.64 & EF212844 & EF212856 & - & - \\
\hline Gnomonia fragariae & $\mathrm{UN} 22$ & EF212833 & EF212851 & - & - \\
\hline Gnomonia fragariae & F 5.6 & EF212840 & EF212855 & - & - \\
\hline Gnomonia fragariae & F 1.4 & - & EF212853 & - & - \\
\hline Gnomonia fragariae & M1 & EF212834 & EF212852 & - & - \\
\hline Gnomonia fragariae & GF2 & EF212832 & $\mathrm{EF} 212850$ & - & - \\
\hline Gnomonia fragariae & SER1 & EF212841 & EF212854 & - & - \\
\hline Gnomonia fragariae & SER7 & EF212843 & - & - & - \\
\hline Gnomonia fragariae & SER4 & EF212842 & - & - & - \\
\hline Gnomonia fragariae & L56 & EF212838 & - & - & - \\
\hline Gnomonia fragariae & $\mathrm{O} 3$ & EF212837 & - & - & - \\
\hline Gnomonia fragariae & $\mathrm{S} 12$ & EF212836 & - & - & - \\
\hline Gnomonia fragariae & S7 & EF212835 & - & - & - \\
\hline Gnomonia gnomon & CBS 829.79 & AY818957 & AY818964 & - & EU221905 \\
\hline Gnomonia gnomon & CBS 199.53 & AY818956 & AF408361 & DQ470922 & EU221885 \\
\hline Gnomonia rosae & CBS 850.79 & EF212846 & EF212858 & - & - \\
\hline Gnomonia rostellata & AR 3533 & JF681962 & - & - & - \\
\hline Gnomonia rostellata & AR 3987 & JF681963 & - & - & - \\
\hline Gnomonia rubi & CBS 102.72 & EF212847 & EF212859 & - & - \\
\hline Hapalocystis berkeleyi & AR 3851 & - & EU683069 & - & - \\
\hline Hapalocystis berkeleyi & AR 3870 & JF681958 & - & - & - \\
\hline Hapalocystis berkeleyi & MFLUCC 13-0662 & - & KP744486 & - & - \\
\hline Hapalocystis occidentalis & WU 24705 & - & AY616231 & - & - \\
\hline Hercospora tiliae & AR 3526 & - & AF408365 & - & - \\
\hline Italiomyces centaureii & MFLU 16-2866 & KY523478 & KY523494 & - & - \\
\hline Italiomyces centaureii & MFLU 16-2867 & KY523477 & KY523495 & - & - \\
\hline Italiomyces centaureii & MFLUCC 14-0849 & KY523476 & KY523496 & - & - \\
\hline Lamproconium desmazieri & MFLUCC 15-0871 & KX430136 & KX430137 & - & - \\
\hline Lamproconium desmazieri & MFLUCC 14-1047 & KX430132 & KX430133 & - & - \\
\hline Lamproconium desmazieri & MFLUCC 15-0870 & KX430134 & KX430135 & - & - \\
\hline Lamproconium desmazieri & MFLUCC 15-0873 & KX430140 & KX430141 & - & - \\
\hline Ophiognomonia rubi-idaei & BPI 877638 & EU254938 & - & - & - \\
\hline Ranulospora alnii & MFLUCC 13-0793 & KY523479 & KY523497 & - & - \\
\hline Ranulospora alnii & MFLUCC 13-0793A & KY523480 & KY523498 & - & - \\
\hline Ranulospora alnii & MFLU 16-2868 & KY523481 & KY523499 & - & - \\
\hline Rossmania ukurunduensis & AR 3484 & - & EU683075 & - & - \\
\hline Sillia italica & MFLU 16-0056A & KY523484 & KY397949 & - & - \\
\hline
\end{tabular}


Table 1. continued. Isolates used in the analyses and their GenBank and culture accession numbers. Newly generated sequences are in bold.

\begin{tabular}{llllll}
\hline \multicolumn{1}{c}{ Taxa } & \multicolumn{1}{c}{$\begin{array}{c}\text { Culture accession } \\
\text { numbers }\end{array}$} & \multicolumn{1}{c}{ ITS } & \multicolumn{2}{c}{ LenBank accession numbers } \\
& \multicolumn{1}{c}{ LSU } & RPB2 & TEF \\
\hline Sillia italica & CBU 16-0056B & KY523485 & - & - & - \\
Sillia ferruginea & MFLU 16-2864 & KF681959 & EU683076 & - & - \\
Sillia karstenii & MFLU 16-2865 & KY523483 & KY523500 & KY501636 & - \\
Sillia karstenii & CBS 117025 & EU039982 & EU039993 & KF570173 & - \\
Stegonsporium acerophilum & CBS 121883 & JX517290 & JX517299 & KF570196 & - \\
Stilbospora macrosperma & MFLU 16-2860 & KY523486 & KY523502 & - & - \\
Sydowiella centaureii & MFLU 16-2858 & KY523487 & KY523503 & - & - \\
Sydowiella centaureii & CBS 813.79 & - & EU683077 & - & - \\
Sydowiella depressula & CBS 125530 & JF681956 & EU683078 & - & - \\
Sydowiella fenestrans & MFLUCC 13-0665 & - & KY523504 & - & - \\
Sydowiella urticola & MFLU 16-1265 & KY523488 & KY523505 & - & - \\
Tenuiappendicula alnicola & MFLU 16-2865 & KY523489 & KY523506 & - & - \\
Tenuiappendicula alnicola & MFLUCC 16-1452 & KY523490 & KY523507 & - & - \\
Tenuiappendicula alnicola & CFCC 89622 & KR045698 & AF362564 & KU710944 & KU710911 \\
Valsa ambiens & AR 4022 & JF681960 & - & - & - \\
Winterella aurantiaca & & & & & - \\
\hline
\end{tabular}

search option with TBR branch swapping and 1000 random sequence additions. MaxTrees were set up to 1000 , branches of zero length were collapsed and all multiple parsimonious trees were saved. Tree length (TL), consistency index (CI), retention index (RI), rescaled consistency index (RC), homoplasy index (HI), and log likelihood (-ln L) (HKY model) were calculated for trees generated under optimality criteria. The robustness of the most parsimonious trees was evaluated by 1000 bootstrap replications resulting from maximum parsimony analysis, each with ten replicates of random step-wise addition of taxa (Felsenstein 1985). The Kishino-Hasegawa tests (Kishino \& Hasegawa 1989) were performed to determine whether the trees inferred under optimality criteria were significantly different. Trees were figured in Treeview (Page 1996). The final alignments and the trees obtained were deposited in TreeBASE (http://purl.org/phylo/treebase/phylows/study/

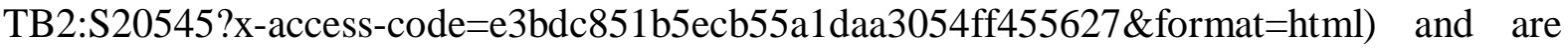
available under study accession no. S20545.

\section{Results and Discussion}

The phylogenetic analyses were carried out using combined LSU, ITS, RPB2 and TEF sequence data from taxa of Diaporthales as listed in Table 1. Taxon sampling was based on GenBank blast searches and recent publications (Morocko 2006, Kruys \& Castlebury 2012, Maharachchikumbura et al. 2015, 2016). The final multiple alignment data comprised 76 taxa and 3283 characters including gaps. Overall, the topologies obtained from the different phylogenetic methods and parameters were congruent and the best scoring RAxML tree is illustrated together with bootstrap support values $(\mathrm{TL}=3526, \mathrm{CI}=0.664, \mathrm{RI}=0.853, \mathrm{HI}=$ $0.336, \mathrm{RC}=0.567$ ) in Fig. 1. Maximum likelihood analysis based on 1000 bootstrap replicates, yielded a tree with the likelihood value of $\ln :-19847.102701$ with the following model parameters: alpha: 0.779450, П(A): 0.238610, П(C): 0.265774, $(\mathrm{G}): 0.286696$, and П(Т): 0.20892 .

The separation of Sydowiellaceae from other diaporthalean taxa (Fig. 1) is wellsupported and Sydowiellaceae comprises 14 clades (Clades A-N) representative of the generic placement. The type genus Sydowiella (Clade N) forms a moderately-supported clade within the family and here we introduce a new species $S$. centaureii which is morphologically and phylogenetically distinct. The phylogeny indicates that Tenuiappendicula (Clade L) constitutes an independent monophyletic clade with high statistical support, while Chapeckia 
(Clade M) has a sister relationship to Tenuiappendicula, but is phylogenetically distinct (Fig. 1). Chapeckia and Tenuiappendicula species have pseudostromata with very thin peridia and 1-septate, oval ascospores. The asexual morph of Tenuiappendicula is illustrated here and ITS, LSU sequences derived from conidiomatal DNA are similar to the sexual morph sequences. A new genus Italiomyces (Clade K) is introduced, with I. centaureii as the type species. Italiomyces differs from other genera in Sydowiellaceae in having mostly biguttulate, rarely 4-guttulate ascospores, with minute, spine-like apical appendages at both ends. The new genus Alborbis (Clade I) is introduced to accommodate Cryptodiaporthe galericulata which is morphologically and phylogenetically distinct from the type species of Cryptodiaporthe. This taxonomic circumscription of $C$. galericulata was suggested by Kruys $\&$ Castlebury (2012). Hence, we introduce Alborbis to accommodate Cryptodiaporthe galericulata with a new combination as Alborbis galericulata. Alborbis is sister to Cainiella (Clade $\mathrm{J}$ ) and the new genus Ranulospora (Clade $\mathrm{H}$ ), typified by $R$. alnii, is introduced as sister to Alborbis and Cainiella. Sillia (Clade F) and Tortilispora (Clade G) both have cylindrical asci containing long, filiform ascospores with pointed ends. Tortilispora, however, has a diaporthoid configuration of ascomata with 4-spored asci and septate paraphyses, while Sillia has a diatrypoid configuration of ascomata with 8-spored asci and aseptate paraphyses (Tibpromma et al. 2017). This morphological segregation is also supported in the phylogeny (Fig. 1). Reid \& Booth (1987) studied Tortilispora (= Winterella), but further collections are needed together with support from molecular data as our assumption is based on only a single species. A new Sillia species, S. karstenii is introduced, as it constitutes a highly-supported subclade and is phylogenetically apart from other species (Fig. 1). The new species also differs from other Sillia species in having a very poorly developed stroma and ascospores with pointed ends (Fig. 15). A new genus Breviappendix (Clade E) is introduced to accommodate Gnomonia rubi and G. rostellata which were previously accommodated in Gnomoniaceae. Breviappendix forms a distinct clade in the family with moderate support. Breviappendix rosae, B. rostellata and B. rubi are introduced as new combinations for Gnomonia rosae, G. rostellata and G. rubi respectively and Breviappendix rubi is designated as the type species of the genus. A new genus Paragnomonia (Clade D) is introduced to accommodate Gnomonia fragariae which was previously placed in Gnomoniaceae. The placement in Sydowiellaceae was also noted by Mejía et al. (2011). Calosporella (Clade A) forms a well-supported separate clade as a wellresolved genus. This monotypic genus was recently revisited by Voglmayr \& Jaklitsch (2014). Calosporella is sister to Rossmania (Clade B) which is represented by $R$. ukurunduensis. Hapalocystis (Clade C) does not have any support and Hapalocystis occidentalis is basal to all other Hapalocystis berkeleyi strains. Hapalocystis berkeleyi and $H$. occidentalis are morphologically similar, but differ primarily in spore ornamentation and size of appendages. Our phylogeny also supports the concept that Hapalocystis occidentalis can be considered as a separate species from $H$. berkeleyi based on morphological differences mentioned and phylogenetic support for this has been reported in other studies (Jeewon et al. 2002).

Figure 1 - Consensus tree resulting from a maximum likelihood analysis of combined LSU, ITS, RPB2 and TEF sequence alignment of genera of Diaporthales. genera in Sydowiellaceae are indicated in pink and green blocks while families are indicated in ash-coloured blocks.. Maximum parsimony bootstrap support (MPB above 50\%), RAxML bootstrap support (MLB above $50 \%$ ), and Bayesian posterior probabilities (PP above 0.9) are given at the nodes (MPB/MLB/PP). The scale bar represents the expected number of changes per site. The tree is rooted to Gnomonia gnomon (CBS 829.79; CBS 199.53). Ex-type strains are in bold and new strains are in blue bold. 


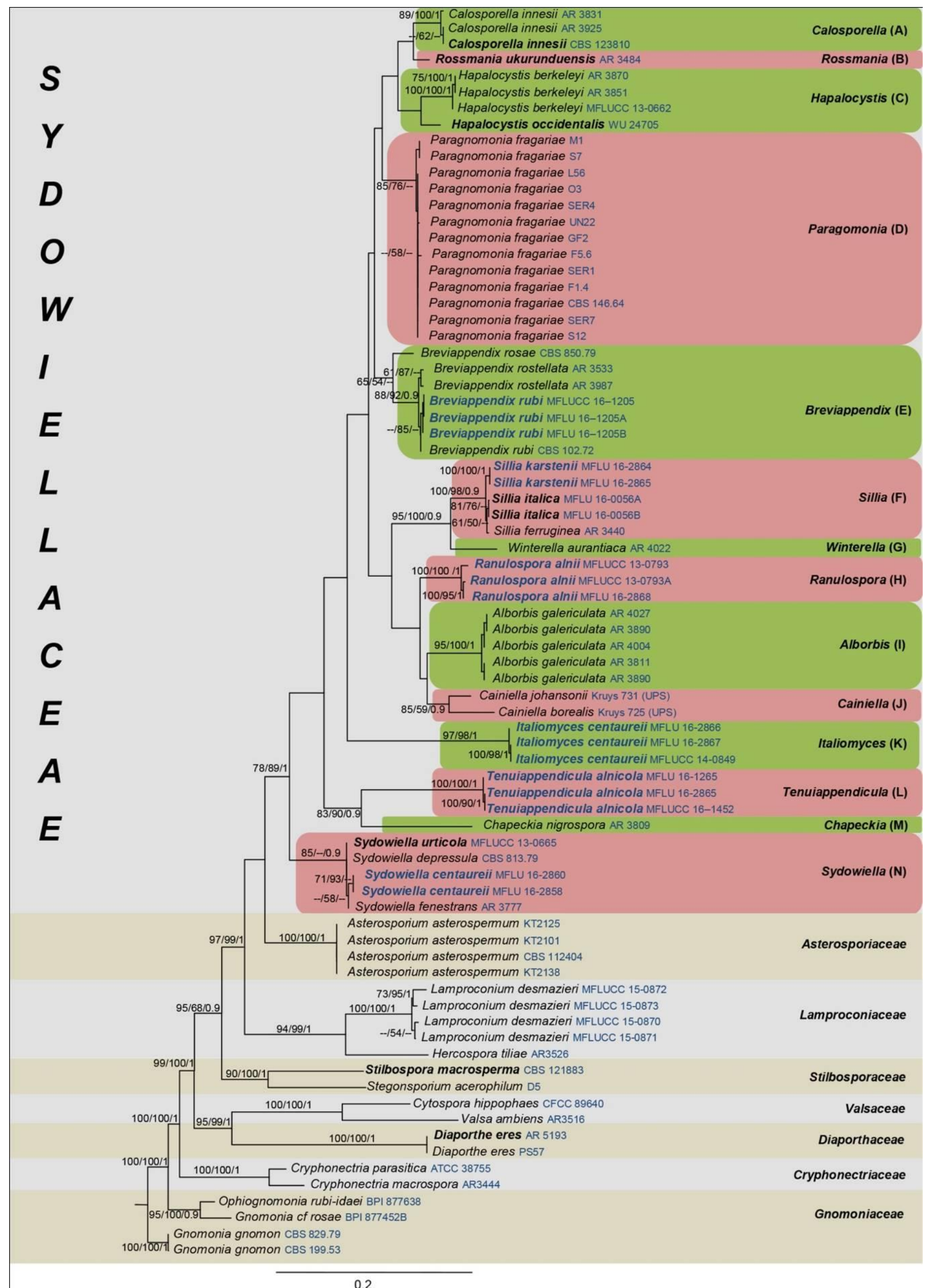




\section{Taxonomy}

Sydowiellaceae Lar.N. Vassiljeva, Pirenomits. Lokuloaskomits. Severa Dal'nego Vostoka (Leningrad): 210 (1987)

Saprobic on dead branches of deciduous trees or parasitic on both living and dead leaves and petioles of temperate shrubs. Sexual morph - Stromata present or absent. If present, erumpent through cracks, roughly circular, pseudoparenchymatous to prosenchymatous with few ascomata in a stroma, erumpent, sometimes with simple to complex stellate splits in the substrate, with poorly to well-developed black lines around the stroma and internal eustromatic tissues with thick-walled, glassy, hyaline cells of textura globulosa to textura angularis around the basal regions of ascomata and dark brown-walled cells of textura globulosa to textura angularis around the ostioles, sometimes becoming rouge in $10 \% \mathrm{KOH}$. If pseudostromatic, pseudostromata pustulate on surface, circular, containing up to 20 ascomata. Ectostromatic disc erumpent, conspicuous, white to light grey, becoming dark at maturity, fusoid to circular, containing 1-20 distinctly protruding ostioles. Entostroma crumbly, brownish. Ascomata scattered, solitary or aggregated, erumpent to immersed, globose to subglobose, coriaceous, brown to black, ostiolate, papillate. Papilla wide, erumpent from the substrate, internal wall covered by hyaline, filamentous periphyses. Peridium comprising outer layer of brown-walled cells of textura angularis to globulosa and inner layer of, hyaline cells of textura angularis. Hamathecium comprising cellular or filamentous, hyaline, septate or aseptate paraphyses or aparaphysate. Asci 8-spored, unitunicate, cylindrical to cylindro-clavate or broadly fusoid, short pedicellate, apex blunt, with J-, apical ring or without apical ring, sometimes apex wide and flat, with multi-layered, $\mathrm{J}-$, apical ring. Ascospores overlapping uniseriate, biseriate to triseriate, kidney-shaped, filamentous, oval or ellipsoidal, hyaline or initially hyaline, becoming brown with age, 0-11septate, slightly constricted or not constricted at the septum, thick and smooth-walled, sometimes multi-guttulate, sometimes hyaline, with tapering bipolar appendages or appendages basal only. Asexual morph - Coelomycetous. Conidiomata pycnidia, superficial to erumpent, aggregated 3-5 in a single group, globose, orange to brown, conidiomatal wall thick-walled, with orange cells of textura angularis. Conidiophores annelidic, branched, hyaline, short, few conidiogenous cells arising from a single conidiophore, attached to conidiomatal wall. Conidiogenous cells cylindrical, hyaline, bottle-shaped, septate, pointedends, phialidic. Conidia ellipsoid or cylindroid, unicellular or multi-septate, hyaline or brown, smooth-walled.

Type genus - Sydowiella Petr.

Notes - Barr (1978) placed Sydowiella in the family Pseudovalsaceae (subfamily Pseudovalsoideae, tribe Ditopelleae) based on its morphological distinctiveness. There have been also a few suggestions to place Sydowiella in Gnomoniaceae. However, Monad (1983), did not accept the placement of Sydowiella in Gnomoniaceae because of absence of stromatic tissues. Vasilyeva (1987) introduced the family Sydowiellaceae based on Sydowiella fenestrans (Duby) Petr. and this family was accommodated in the order Ceratostomatales. Rossman et al. (2007) included Chapeckia, Hapalocystis, Rossmania, Sillia, and Stegophora in Sydowiellaceae. Sogonov et al. (2008) noted a close affinity of Lambro and Uleoporthe with this family, while Kruys \& Castlebury (2012) analyzed ITS sequence data for genera in the family. The phylogenetic position of Cainiella in Sydowiellaceae was resolved and Winterella was also included in the family based on molecular data. Calosporella was introduced and placed in Sydowiellaceae to accommodate Prosthecium innesii by Voglmayr \& Jaklitsch (2014). 


\section{List of accepted genera and species in Sydowiellaceae}

Based on Index Fungorum 2017, Maharachchikumbura et al. (2015, 2016), Mejia et al. (2008), MycoBank (2017), Tibpromma et al. (2017)

Alborbis Senan. \& K.D. Hyde

Alborbis galericulata (Tul. \& C. Tul.)

Senan. \& K.D. Hyde

Breviappendix Senan. \& K.D. Hyde

Breviappendix rosae (Fuckel) Senan. \&

K.D. Hyde

Breviappendix rostellata (Fr.) Senan. \&

K.D. Hyde

Breviappendix rubi (Rehm) Senan. \& K.D. Hyde

Cainiella E. Müll.

Cainiella borealis M.E. Barr

Cainiella johansonii (Rehm) E. Müll.

Calosporella J. Schröt.

Calosporella innesii (Curr.) J. Schröt.

Chapeckia M.E. Barr

Chapeckia nigrospora (Peck) M.E. Barr

Chapeckia ribesia (Sacc. \& Scalia) M.E.

Barr

Hapalocystis Auersw. ex Fuckel

Hapalocystis berkeleyi Auersw. ex Fuckel

Hapalocystis bicaudata Fuckel

Hapalocystis corni (Wehm.) M.E. Barr

Hapalocystis corticalis (Schwein.) M.E.

Barr

Hapalocystis occidentalis Jaklitsch \& Voglmayr

Hapalocystis ulmi (Wehm.) M.E. Barr

Hapalocystis vexans Speg.

Italiomyces Senan., Camporesi \& K.D. Hyde

Italiomyces centaureii Senan., Camporesi \& K.D. Hyde

Lambro Racib. $=$ Stegophora Syd. \& P. Syd

Lambro insignis Racib.

Lambro symploci G.C. Zhao \& R.L. Zhao

\section{Paragnomonia}

Paragnomonia fragariae (Kleb.) Senan. \& K.D. Hyde

Ranulospora Senan., Camporesi \& K.D. Hyde

Ranulospora alnii Senan., Camporesi \& K.D. Hyde

Rossmania Lar.N. Vassiljeva

Rossmania aculeata (Petch) Lar.N.

Vassiljeva

Rossmania ukurunduensis Lar.N. Vassiljeva

Sillia P. Karst.

Sillia albofusca Höhn.

Sillia biformis Rick

Sillia celastrina R. Rao

Sillia ferruginea (Pers.) P. Karst.

Sillia italica de Silva, Camporesi \& K.D.

Hyde

Sillia kamatii Tilak, S.B. Kale \& S.V.S.

Kale

Sillia karstenii Senan., Camporesi \& K.D.

Hyde

Sillia theae Hara

Tenuiappendicula Senan., Camporesi \& K.D. Hyde

Tenuiappendicula alnicola Senan., Camporesi \& K.D. Hyde

Tortilispora (Sacc.) Senan. \& K.D. Hyde $=$ Cryptosporella Sacc.

Tortilispora albofusca (Cooke \& Ellis)

Senan. \& K.D. Hyde

Tortilispora aurantiaca (Wehm.) Senan. \& K.D. Hyde

Tortilispora cinctula (Cooke \& Peck)

Senan. \& K.D. Hyde 


\section{Key to genera within the family Sydowiellaceae}

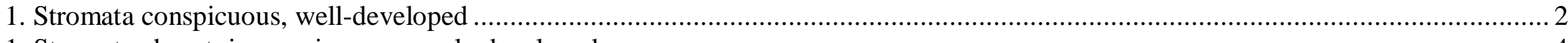

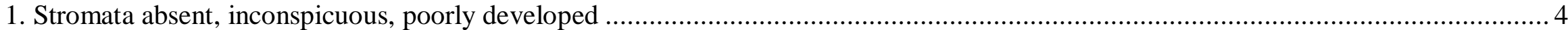

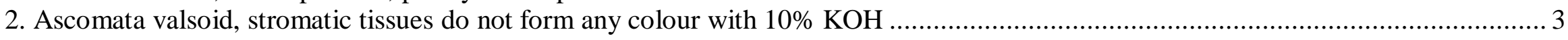

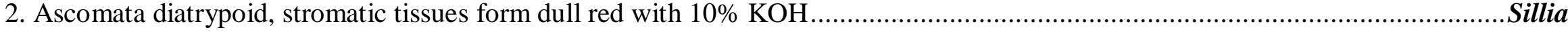

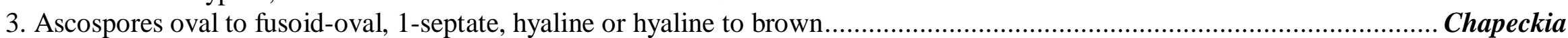

3. Ascospores fusiform to ellipsoid with long filiform basal cell, 2-septate, hyaline.....................................................Ranulospora

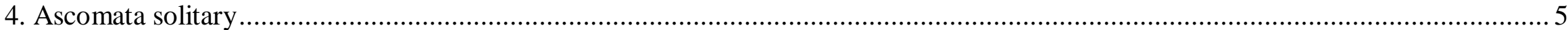

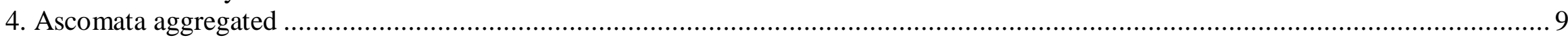

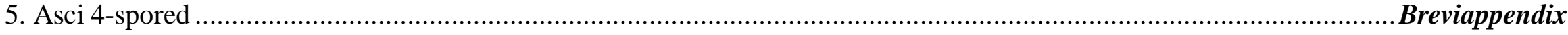

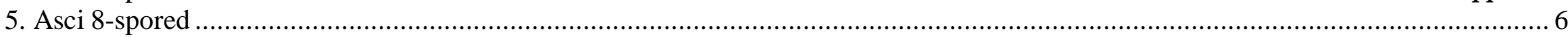

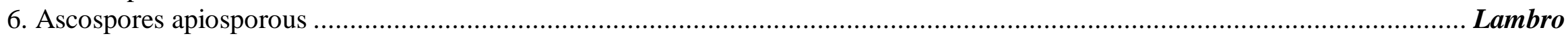

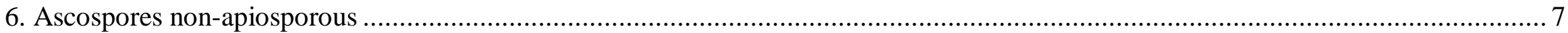

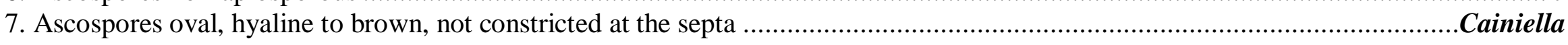

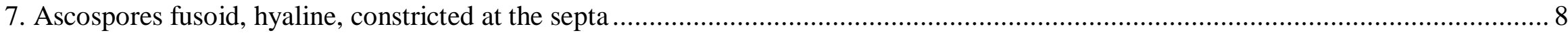

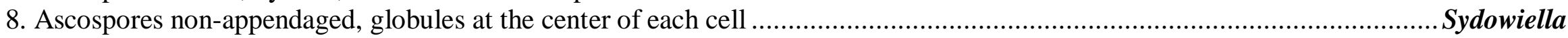

8. Ascospores appendaged, globules at the septa of the spore ….................................................................................Itiomyces

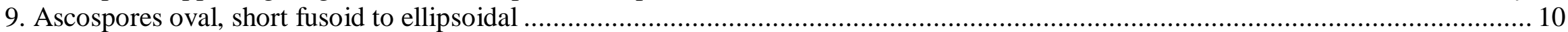

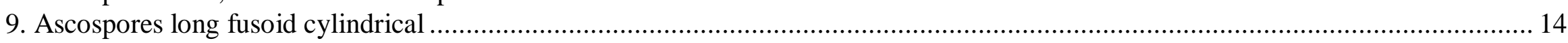

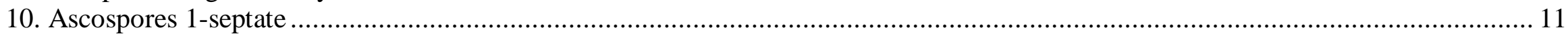

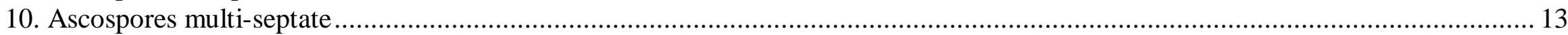

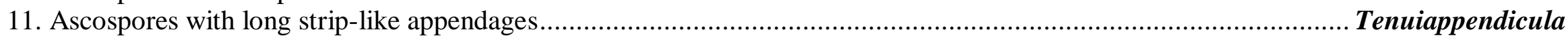

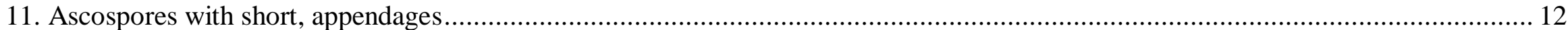

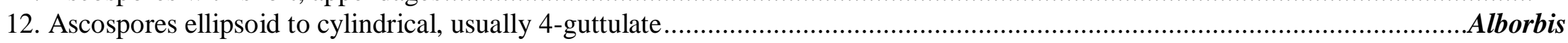

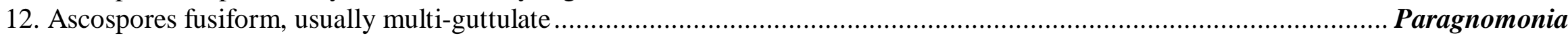

13. Ascospores hyaline to brown, ellipsoidal with broadly rounded ends, $1-3$-septate, constricted at the septa .......................Hapalocystis

13. Ascospores hyaline, fusoid to oblong, 3-4-eusepta, not constricted at the septa ..............................................................osporella

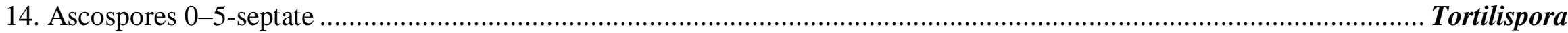

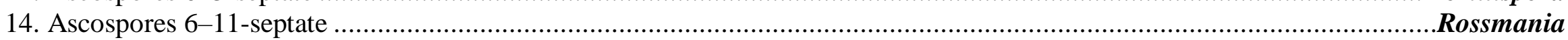


Sydowiella Petr., Annls mycol. 21(1/2): 30 (1923)

Saprobic or pathogenic on plants. Sexual morph - Ascomata solitary, scattered, erumpent to immersed, globose, brown, papillate, ostiolate. Papilla short and wide, comprising brown, elongated cells. Peridium comprising an inner layer of thin-walled, hyaline cells of textura angularis and outer layer of thick-walled, brown cells of textura angularis. Hamathecium comprising cellular, septate, hyaline paraphyses. Asci 8-spored, unitunicate, cylindrical, shortpedicellate, apex blunt, with a J-, apical ring. Ascospores overlapping uniseriate, oval to ellipsoid, 1-median septate, hyaline. Asexual morph - Undetermined.

Type species - Sydowiella fenestrans (Duby) Petr.

Basionym - Sphaeria fenestrans Duby

Notes - Sydowiella is characterized by solitarily, erumpent ascomata without stromatic tissues, a melanconis-like hamathecium, and 2-celled ascospores (Kobayashi 1970). The type species Sydowiella fenestrans was illustrated in Maharachchikumbura et al. (2016). Sydowiella comprises ten species (Index Fungorum 2017): S. ambigua (Mouton) Munk, S. azukiae I. Hino \& Katum., S. dakotensis Petr., S. depressula (P. Karst.) M.E. Barr, S. fenestrans (Duby) Petr., S. indica Bilgrami \& Rizwi, S. juncina Spooner, S. stellatifolii Marinc. et al. and S. uticola Senan. et al. Here we introduce an additional species.

Sydowiella centaureii Senan., Camporesi \& K.D. Hyde, sp. nov.

Index Fungorum number - IF552715, Facesoffungi number - FoF $2834 \quad$ Fig. 2

Etymology - Name referring to the host genus Centaurea.

Holotype - MFLU 16-2858

Saprobic on dead branches of Centaurea L. Sexual morph - Stromata pseudoparenchymatous, comprising outer dark brown to black line made by hyphal cells and inner light yellow to hyaline, large cells of textura globosa. Ascomata 450-510 $\mu \mathrm{m}$ high, $280-355 \mu \mathrm{m}$ diameter $(\bar{x}=504 \times 314$ $\mu \mathrm{m}, \mathrm{n}=20$ ), scattered, solitary, immersed to erumpent, erect or slanted, globose to subglobose, coriaceous, black, papillate, ostiolate. Papilla $185-195 \mu \mathrm{m}$ high, $110-120 \mu \mathrm{m}$ wide $(\bar{x}=190 \times 115$ $\mu \mathrm{m}, \mathrm{n}=20)$, internally covered with hyaline periphyses. Peridium $15-25 \mu \mathrm{m}$ wide $(\bar{x}=21 \mu \mathrm{m}, \mathrm{n}=$ 20), comprising inner layer of hyaline, thin-walled, compressed cells of textura angularis and outer layer of dark brown, thick-walled, cells of textura angularis. Hamathecium comprising 4-7 $\mu \mathrm{m}(\bar{x}$ $=6 \mu \mathrm{m}, \mathrm{n}=20)$ wide, septate, cellular paraphyses. Asci 85-110 $\times 10-15 \mu \mathrm{m}(\bar{x}=102 \times 14 \mu \mathrm{m}, \mathrm{n}$ = 20), 8-spored, unitunicate, cylindrical, short-pedicellate, with distinct, bilobed, J- apical ring. Ascospores $10-15 \times 5-8 \mu \mathrm{m}(\bar{x}=13 \times 6.8 \mu \mathrm{m}, \mathrm{n}=20)$, overlapping uniseriate, oval to widely fusiform, hyaline, 1-septate, slightly constricted at the septum, with a large guttule in each cell. Asexual morph - Undetermined.

Material examined - ITALY, Province of Forlì-Cesena, Fiumicello di Premilcuore, dead stem of Centaurea sp. (Asteraceae), 2 April 2014, Erio Camporesi, IT 1792 (MFLU 16-2858, holotype; ibid $\mathrm{BBH}$, isotype).

Notes - Sydowiella juncina and S. stellatifolii were collected on Juncus effusus L. and Brabejum stellatifolium L. respectively, and are possibly host-specific species. Sydowiella ambigua and $S$. azukiae form aggregated ascomata, while $S$. centaureii has solitary ascomata. Sydowiella centaureii differs from $S$. fenestrans and $S$. urticola in having large ascomata immersed within distinct stromatic tissues, surrounded by a layer of dark cells and small asci and ascospores (Maharachchikumbura et al. 2016, Index Fungorum 2017). However, we could not obtain a culture from this fungus and we extracted DNA directly from the ascomata.

Alborbis Senan. \& K.D. Hyde, gen. nov.

Index Fungorum number - IF552717, Facesoffungi number - FoF 2835

Etymology - based on Latin words "alba" and "orbis", referring to the ascomata opening to the host surface through a whitish disk.

Saprobic on dead stems. Sexual morph - Ectostromatic disc appearing as pustulate swellings, small, whitish, usually containing several ascomata. Ascomata immersed, aggregated, globose to 

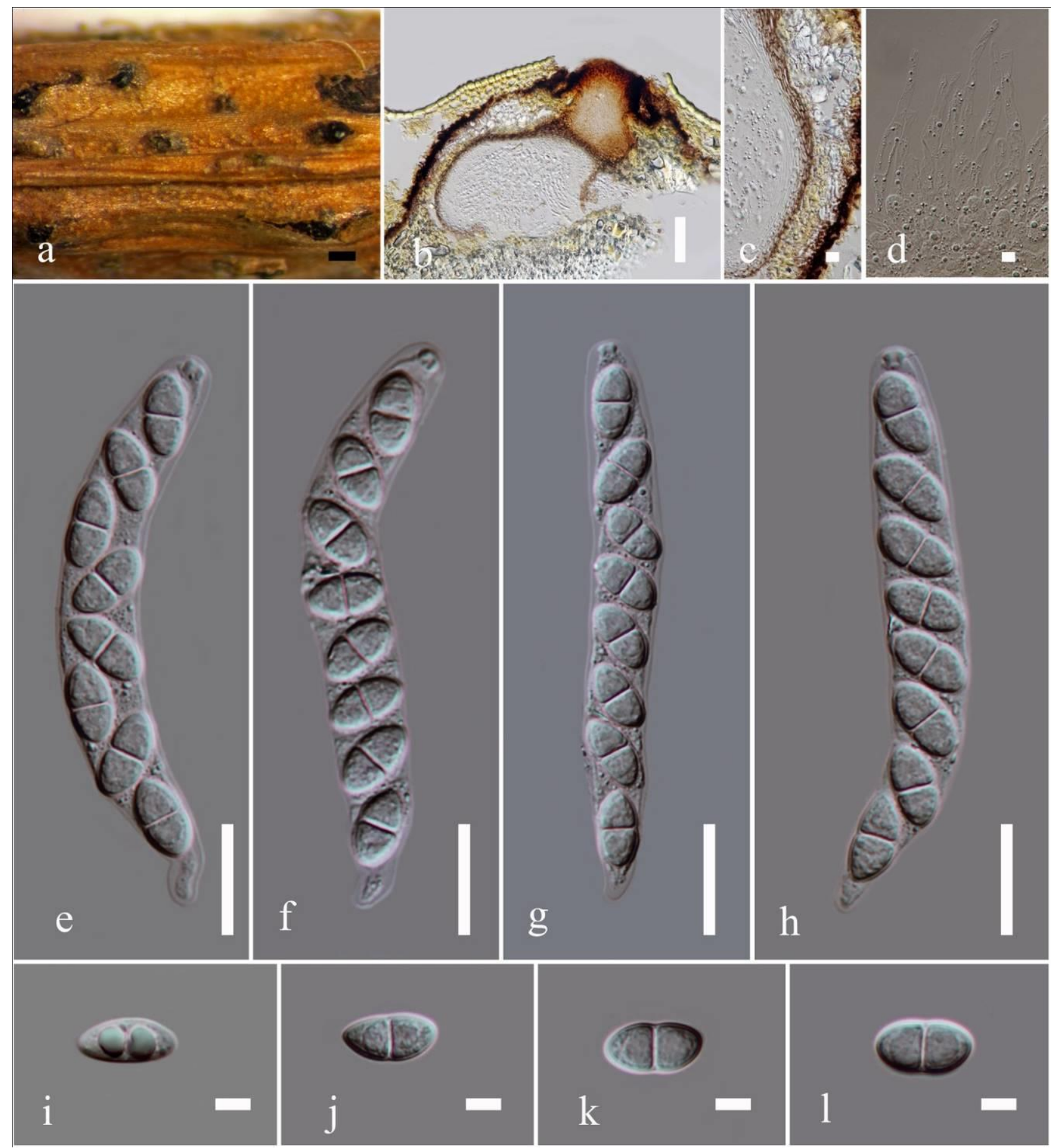

Figure 2 - Sydowiella centaureii (holotype) a Appearance of ascomata on substrate. b Vertical section of ascoma. c Peridium. d Paraphyses. e-h Asci. i-1 Ascospores. Scale bars $-\mathrm{a}=200 \mu \mathrm{m}, \mathrm{b}=$ $100 \mu \mathrm{m}, \mathrm{c}=10 \mu \mathrm{m}, \mathrm{d}=5 \mu \mathrm{m}, \mathrm{e}-\mathrm{h}=20 \mu \mathrm{m}, \mathrm{i}-1=5 \mu \mathrm{m}$.

subglobose, coriaceous, black, ostiolate. Ostiolar canal scarcely emergent, separate, opening to ectostromatic disc by a wide ostiole, internally covered by hyaline, filamentous periphyses. Peridium comprising thick-walled, dark brown cells of textura angularis. Asci 8-spored, unitunicate, cylindrical to clavate, apex rounded with $\mathrm{J}$-, apical ring. Ascospores overlapping uniseriate to biseriate, fusiform, 1-septate, sometimes with apical and basal appendages. Asexual morph - Undetermined.

Type species - Alborbis galericulata (Tul. \& C. Tul.) Senan. \& K.D. Hyde 


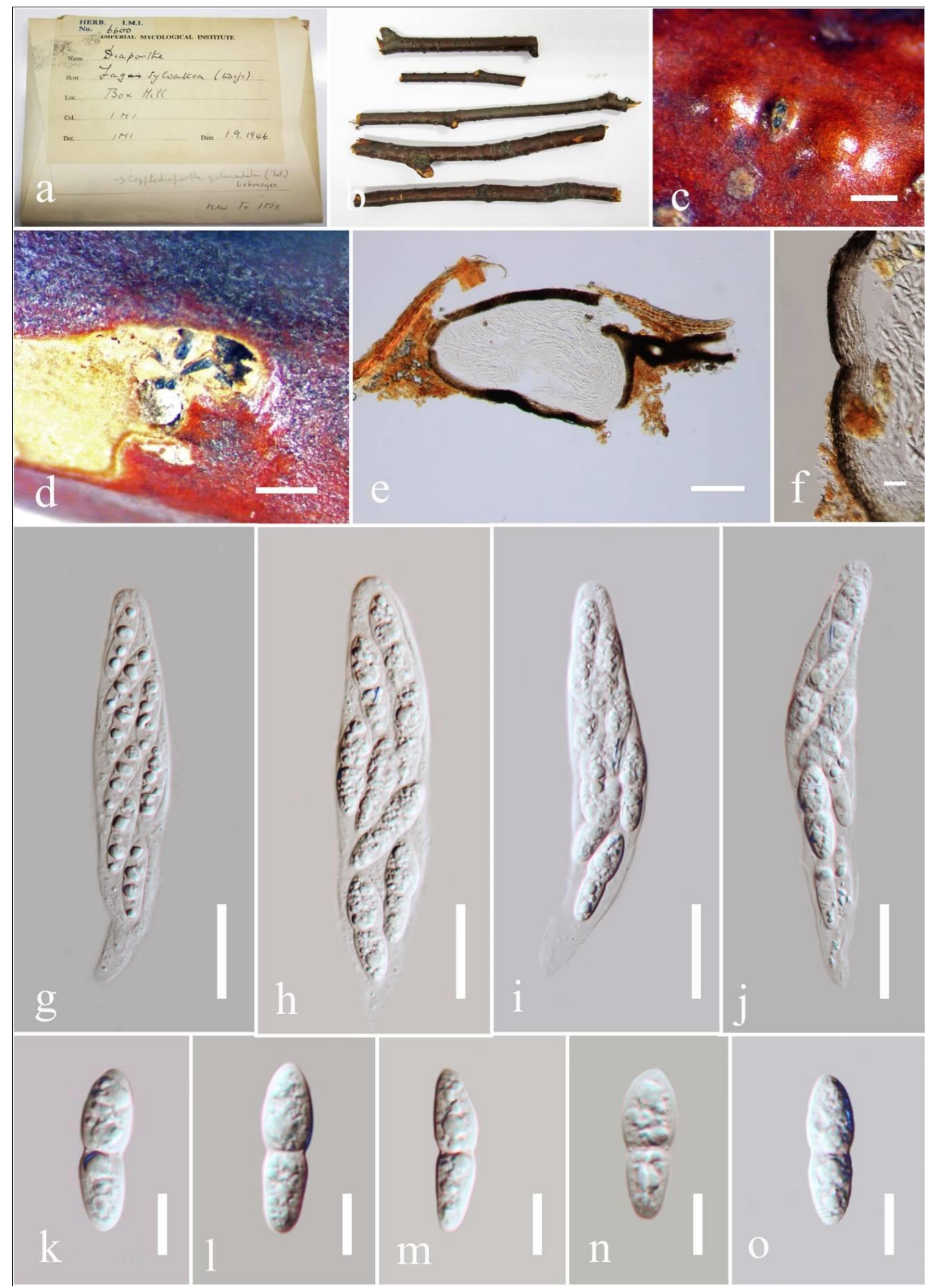

Figure 3 - Alborbis galericulata (IMI 6600). a Herbarium packet. b Herbarium specimen. c Appearance of ascomata on host surface. $d$ Horizontal section of ascomata through the ectostromatic disc. e Vertical cross section through the ascomata. f Peridium. g-j Asci. k-o Ascospores. Scale bars $-\mathrm{c}, \mathrm{d}=500 \mu \mathrm{m}, \mathrm{e}=200 \mu \mathrm{m}, \mathrm{f}-\mathrm{j}=20 \mu \mathrm{m}, \mathrm{k}-\mathrm{o}=10 \mu \mathrm{m}$. 
Alborbis galericulata (Tul. \& C. Tul.) Senan. \& K.D. Hyde, comb. nov.

Index Fungorum number - IF552718, Facesoffungi number - FoF 2836

Fig. 3

Basionym - Valsa galericulata Tul. \& C. Tul., Select. fung. carpol. (Paris) 2: 203 (1863)

Holotype - NYBG 02929068

Saprobic on dead stems of Fagus sylvatica L. Sexual morph - Appearing on the surface as small, pustulate swellings, with a central stellate rupture of the periderm through a white, minute, elliptic to fusoid ectostromatic disk, usually containing 1-6 ascomata. Ascomata 600-950 $\mu \mathrm{m}$ high, 260-450 $\mu \mathrm{m}$ diameter, immersed, aggregated, globose to subglobose, coriaceous, black, ostiolate. Ostiolar canal 375-400 $\mu \mathrm{m}$ high, 80-90 $\mu \mathrm{m}$ wide $(\bar{x}=390 \times 83 \mu \mathrm{m}, \mathrm{n}=20)$, scarcely emergent, separate, opening to host surface by wide ostiole, internally covered by hyaline, filamentous periphyses. Peridium 30-35 $\mu \mathrm{m}$ thick, comprising thick-walled, dark brown cells of textura angularis. Asci $90-110 \times 17-20 \mu \mathrm{m}(\bar{x}=100 \times 19 \mu \mathrm{m}, \mathrm{n}=20)$, 8-spored, unitunicate, cylindrical to clavate, apex rounded with J- apical ring, short-pedicellate. Ascospores $21-26 \times 5-7 \mu \mathrm{m}(\bar{x}=24$ $\times 6 \mu \mathrm{m}, \mathrm{n}=20$ ), biseriate to triseriate, often 4-guttulate, ellipsoid to cylindrical, 1-septate, hyaline, slightly constricted at the septum lacking appendages. Asexual morph - Undetermined.

Material examined - UK, England, Surrey, Box Hill, on twigs of Fagus sylvatica L (Fagaceae), 1 September 1946, IMI 6600.

Notes - Cryptodiaporthe galericulata was introduced by Tulasne \& Tulasne (1863) based on Valsa galericulata. Cryptodiaporthe galericulata has been reported as causing mild canker of Fagus species and saprobic on branches of Fagus sylvatica (Mejía et al. 2011). Wehmeyer (1933) mentioned that Cryptodiaporthe contains a heterogenous group of species. Barr (1978) accommodated Cryptodiaporthe galericulata in family Valsaceae (Tribe Valseae). However, the type species of Cryptodiaporthe is accommodated in Gnomoniaceae based on phylogeny. Mejía et al. (2011) noted that LSU based phylogeny of a fresh collection of C. galericulata (BPI 863767) from the USA, clustered in Sydowiellaceae rather than Gnomoniaceae. Kruys \& Castlebury (2012) placed $C$. galericulata in Sydowiellaceae using ITS sequence data. Combined LSU, ITS, RPB2 and TEF sequence analysis in this study (Fig. 1) also places C. galericulata in Sydowiellaceae. Hence, we exclude $C$. galericulata from Cryptodiaporthe and introduce a new genus Alborbis to accommodate Cryptodiaporthe galericulata.

Breviappendix Senan. \& K.D. Hyde, gen. nov.

Index Fungorum number - IF552719, Facesoffungi number - FoF 2837

Etymology - based on Latin words "appendicibus" and "brevi" referring to the ascospores having short appendages.

Pathogenic on living stems forming cankers. Sexual morph - Ascomata solitary, scattered, erumpent to immersed, globose, coriaceous, brown, papillate, ostiolate. Papilla short, wide, comprising brown, elongated cells, internally covered by hyaline periphyses. Peridium comprising inner layer of thin-walled, hyaline, compressed cells of textura angularis and outer layer of thickwalled, brown, compressed cells of textura angularis. Hamathecium aparaphysate. Asci 8-spored, unitunicate, fusiform, sessile, apex blunt, with J- apical ring. Ascospores biseriate, fusiform to ellipsoid, 1-median septate, hyaline, ends with appendages. Asexual morph - Undetermined.

Type species - Breviappendix rubi (Rehm) Senan., Maharachch. \& K.D. Hyde

Breviappendix rubi (Rehm) Senan., Maharachch. \& K.D. Hyde, comb. nov.

Index Fungorum number - IF552720, Facesoffungi number - FoF 2838

Figs 4, 5

Synonymy - Gnomonia tetraspora var. rubi Rehm, Ascomyceten, fasc. 12: no. 596 (1880)

Gnomonia rubi (Rehm) G. Winter, Hedwigia 26(2): 62 (1885)

Epitype - MFLU 16-2861

Pathogenic on living stems of Rubus ulmifolius Schott. forming cankers. Sexual morph Ascomata 660-760 $\mu \mathrm{m}$ high, 520-535 $\mu \mathrm{m}$ diameter $(\bar{x}=720 \times 530 \mu \mathrm{m}, \mathrm{n}=15)$, solitary, scattered, erumpent to immersed, globose, coriaceous, brown, papillate, ostiolate. Papilla 380-410 $\mu \mathrm{m}$ high, 130-220 $\mu \mathrm{m}$ diameter $(\bar{x}=405 \times 210 \mu \mathrm{m}, \mathrm{n}=15)$, comprising brown, elongate cells, internally 
covered by hyaline periphyses. Peridium $20-35 \mu \mathrm{m}$ wide $(\bar{x}=31 \mu \mathrm{m}, \mathrm{n}=15)$, comprising inner, thin-walled, hyaline, compressed cells of textura angularis and outer, thick-walled, brown, compressed cells of textura angularis. Hamathecium 9-11 $\mu \mathrm{m}$ wide at base $(\bar{x}=10 \mu \mathrm{m}, \mathrm{n}=15)$, 4-5 $\mu \mathrm{m}$ wide at the apex $(\bar{x}=4.8 \mu \mathrm{m}, \mathrm{n}=15)$, comprising thin-walled cellular, septate, paraphyses. Asci 35-45 ×5-10 $\mu \mathrm{m}(\bar{x}=41 \times 7.8 \mu \mathrm{m}, \mathrm{n}=25), 4-(6)$-spored, unitunicate, fusiform, sessile, apex blunt, with a J- apical ring. Ascospores $10-15 \times 2.5-3.5 \mu \mathrm{m}(\bar{x}=13 \times 2.8 \mu \mathrm{m}, \mathrm{n}=$ 25), biseriate, ellipsoid, 1-median septate, slightly constricted at the septa, hyaline, smooth-walled, ends with small, pointed small, setose appendages 1.5-6 $\mu \mathrm{m}$ long. Asexual morph coelomycetous. Conidiomata on MEA sporodochial, developing in 14 days, with a golden-yellow, slimy, pycnidial, superficial, globoid conidial head 150-200 $\mu \mathrm{m}$ wide/diameter $(\bar{x}=180 \mu \mathrm{m}, \mathrm{n}=$ 15). Conidiophores $7-11 \mu \mathrm{m}$ high, $1-3 \mu \mathrm{m}$ diameter $(\bar{x}=9 \times 1.6 \mu \mathrm{m}, \mathrm{n}=15)$, macronematous, cylindrical, short, septate, branched, hyaline, smooth, compactly packed. Conidiogenous cells 10$20 \mu \mathrm{m}$ high, $1-2.5 \mu \mathrm{m}$ diameter $(\bar{x}=14.8 \times 1.7 \mu \mathrm{m}, \mathrm{n}=15)$, phialidic, discrete, vase-shaped, without collarette, hyaline, sometimes branched, aseptate, narrowing towards the apex. Conidia 5.5-7 $\times 1.5-2 \mu \mathrm{m}(\bar{x}=6.5 \times 1.8 \mu \mathrm{m}, \mathrm{n}=15)$, lunar, widest in the centre, pointed at both ends, aseptate, smooth, hyaline, accumulated in a slimy, golden-yellow mass.

Material examined - ITALY, Province of Forlì-Cesena, Santa Sofia, near Corniolo, on dead branch of Rubus ulmifolius Schott (Rosaceae), 25 August 2014, Erio Camporesi, IT 2070 (MFLU 16-2861, epitype designated here), ex-epitype living culture, MFLUCC 16-1205, TBRC; ibid MFLU 16-2862, BBH).

Culture characters - Colonies growing on MEA attaining $1 \mathrm{~cm}$ diameter within 7 days at 18 ${ }^{\circ} \mathrm{C}$, rich in aerial mycelia, woolly, circular, flat, smooth, greyish white.

Notes - Gnomonia rubi was reported as the causal agent of severe stem cankers and dieback of various cultivated and wild Rubus species in both Europe and America (Ellis et al. 1984, Maas et al. 1989, Nordskog et al. 2003). Morphologically G. rubi and G. rostellata (Fr.) Wehm., have similar characters and some authors have synonymized G. rubi under G. rostellata (Monod 1983, Barr 1991). Barr (1978) reported G. rubi as the four-spored form of G. rostellata. Studies have placed Gnomonia rosae, G. rostellata and G. rubi out of Gnomoniaceae as they cluster together with Hapalocystis species (Morocko \& Fatehi 2007). Kruys \& Castlebury (2012) placed G. rubi in Sydowiellaceae based on ITS sequence data. In this study, we observed the same results and hence we introduced a new genus Breviappendix to accommodate G. rosae, G. rostellata and G. rubi and we proposed three new combinations as Breviappendix rosae (IF552798), B. rostellata (IF552799) and $B$. rubi. Breviappendix rubi is designated as the type species and we observed two specimens of Breviappendix rubi (= Gnomonia rubi) from IMI (IMI 252650, IMI 286320). Our fresh collection is morphologically identical to the prologue of Gnomonia rubi and the specimens we observed. However, we could not obtain the type specimen which is probably destroyed. The holotype was collected from Rubus fruticosus L (Rosaceae) in Switzerland and our fresh collection was collected from Rubus ulmifolius Schott in Italy. Considering the morphological similarity and close proximity of collections, we therefore designate our fresh collection as an epitype of Breviappendix rubi (sensu Ariyawansa et al. 2014) and both sexual and asexual morphs are illustrated herein.

Breviappendix rosae (Fuckel) Senan., Maharachch. \& K.D. Hyde, comb. nov. Index Fungorum number - IF552798

Basionym - Gnomonia rosae Fuckel, Jb. nassau. Ver. Naturk. 23-24: 122 (1870) [1869-70]

Breviappendix rostellata (Fr.) Senan., Maharachch. \& K.D. Hyde, comb. nov. Index Fungorum number - IF552799

Basionym - Sphaeria rostellata Fr., Observ. mycol. (Havniae) 1: 178 (1815) 

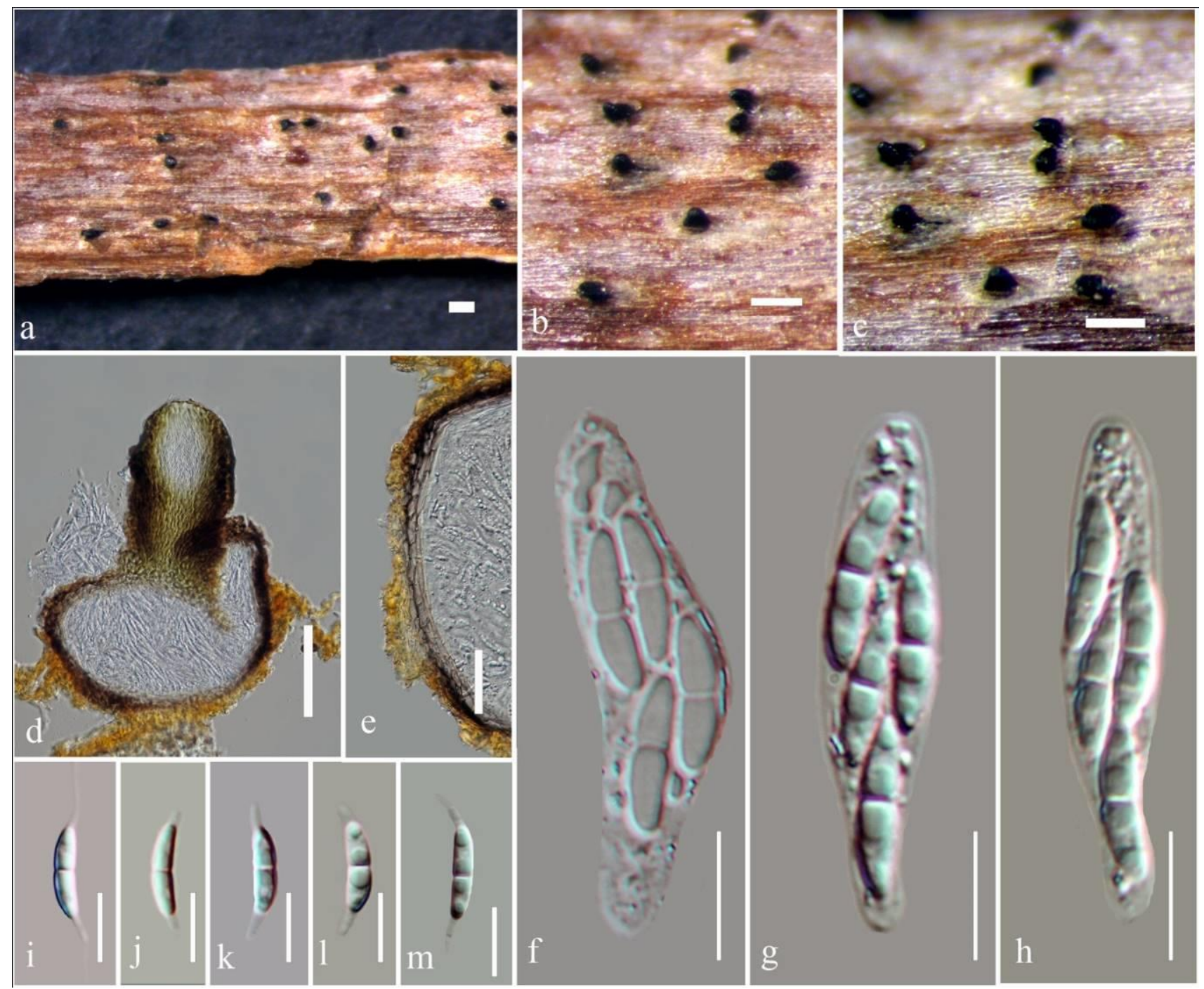

Figure 4 - Breviappendix rubi (MFLU 16-2861, epitype). a-c Appearance of ascomata on substrate. d Vertical section of ascoma. e Peridium. f-h Asci. i-m Ascospores. Scale bars $-\mathrm{a}-\mathrm{c}=$ $200 \mu \mathrm{m}, \mathrm{d}=100 \mu \mathrm{m}, \mathrm{e}=50 \mu \mathrm{m}, \mathrm{f}-\mathrm{m}=10 \mu \mathrm{m}$.

Cainiella E. Müll., Sydowia 10(1-6): 120 (1957) [1956]

Facesoffungi number - FoF2839

Saprobic or parasitic on both living and dead leaves and petioles of temperate shrubs. Sexual morph - Ascomata scattered, solitary or in pairs, erumpent, globose to subglobose, coriaceous, black, ostiolate, papillate. Papilla wide, erumpent, internal wall covered by hyaline, filamentous periphyses. Peridium comprising a few layers of outer brown-walled cells of textura globulosa and inner layer of hyaline cells of textura angularis. Hamathecium comprising filamentous, hyaline, aseptate paraphyses. Asci few in number, 8-spored, unitunicate, cylindro-clavate, short pedicellate, apex wide, flat, with, J- apical ring. Ascospores overlapping uniseriate to biseriate, kidney-shaped or ellipsoidal, guttulate, initially hyaline, greenish or yellowish, becoming brown with age, 1septate, with a thick and smooth epispore. Asexual morph - Undetermined.

Type species - Cainiella johansonii (Rehm) E. Müll.

Cainiella borealis M.E. Barr, Contr. Inst. bot. Univ. Montréal 73: 65 (1959)

Facesoffungi number - FoF2840

Fig. 6

Saprobic or parasitic on both living and dead leaves and petioles of temperate shrubs. Sexual morph - Ascomata 775-1000 $\mu \mathrm{m}$ high, 260-345 $\mu \mathrm{m}$ diameter $(\bar{x}=846 \times 300 \mu \mathrm{m}, \mathrm{n}=15)$, scattered, solitary, erumpent, globose to subglobose, coriaceous, black, ostiolate, papillate. Papilla 400-450 $\mu \mathrm{m}$ high, $120-150 \mu \mathrm{m}$ diameter $(\bar{x}=415 \times 142 \mu \mathrm{m}, \mathrm{n}=20)$, erumpent, internal wall 
covered by hyaline, filamentous periphyses. Peridium $15-25 \mu \mathrm{m}$ wide $(\bar{x}=21 \mu \mathrm{m}, \mathrm{n}=20)$, comprising a few outer layers of brown-walled cells of textura globulosa and inner, hyaline, cells of textura angularis. Hamathecium comprising filamentous, hyaline, aseptate paraphyses. Asci 190-220 $\times 30-40 \mu \mathrm{m}(\bar{x}=204 \times 35 \mu \mathrm{m}, \mathrm{n}=20)$, few in number, 8-spored, unitunicate, cylindroclavate, short pedicellate, apex wide, flat with complex, J-, apical ring. Ascospores 30-35 × 17-20 $\mu \mathrm{m}(\bar{x}=34 \times 19 \mu \mathrm{m}, \mathrm{n}=20)$, biseriate, kidney-shaped, guttulate, hyaline, 1-septate, slightly constricted at the septum, with a thick epispore. Asexual morph - Undetermined.

Material examined - NORWAY, Svalbard and Jan Mayen, Svalbard, Brøgger Peninsula: 6 $\mathrm{km} \mathrm{SE}$ of Ny-Alesund near the bird cliff on the beach, on stems of Cassiope tetragona D. Don (Ericaceae), 12 August 1988, Nils Lundqvist, 17327-d, S-F133018.

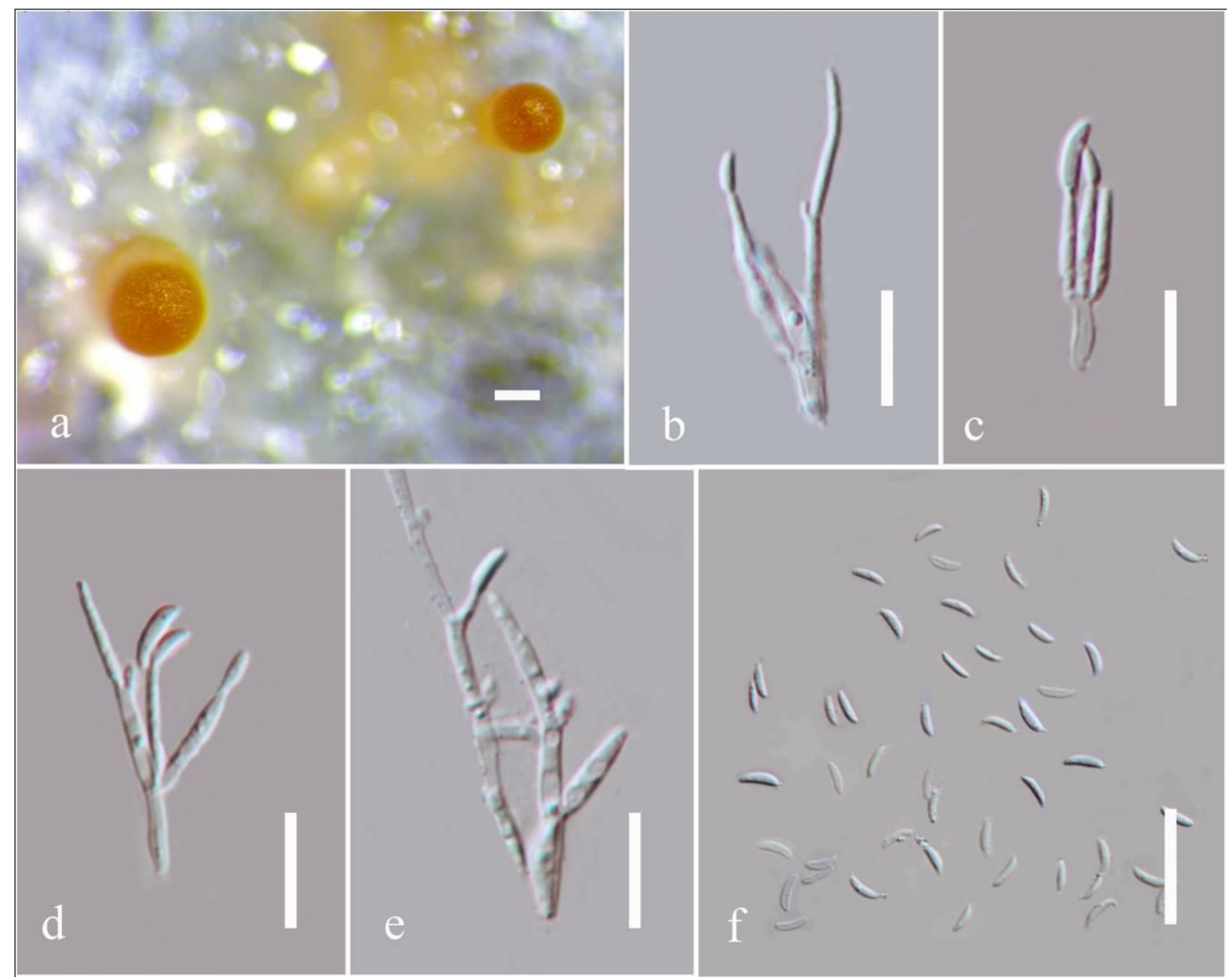

Figure 5 - Asexual morph of Breviappendix rubi (ex-epitype). a Conidiomata on MEA. b-e Conidiophores, conidiogenous cells and conidia. f Conidia. Scale bars $-\mathrm{a}=100 \mu \mathrm{m}, \mathrm{b}-\mathrm{f}=10 \mu \mathrm{m}$.

Notes - The genus Cainiella was introduced by Muller (1957) based on Lizonia johansonii Rehm. This genus is characterized by dark, immersed ascomata, with long-necks, deliquescing paraphyses, as well as asci with a J-, refractive ring and 1-septate ascospores. Cainiella comprises the type species $C$. johansonii (Rehm) E. Müll. and C. borealis. These taxa are associated with arctic dwarf shrubs and prostrate evergreen subshrubs. Initially, this genus was assigned to Sphaeriales (Muller 1956). However, Barr (1959) placed Cainiella in Xylariaceae when she introduced C. borealis. Subsequently Cainiella was placed in Amphisphaeriaceae (Muller \& von Arx 1962), in Cainiaceae (Vasilyeva 1987, 1998) and in Hyponectriaceae (Kirk et al. 2008). Vasilyeva (1998) separated the two species, transferring C. borealis to Sydowiella (Sydowiellaceae) and C. johansonii to Cainia (Cainiaceae). However, Lumbsch \& Huhndorf (2009) placed Cainiella in the family Sordariaceae. 
Kruys \& Castlebury (2012) reported that the taxonomic placement of Cainiella is in Sydowiellaceae based on LSU and ITS sequence data.

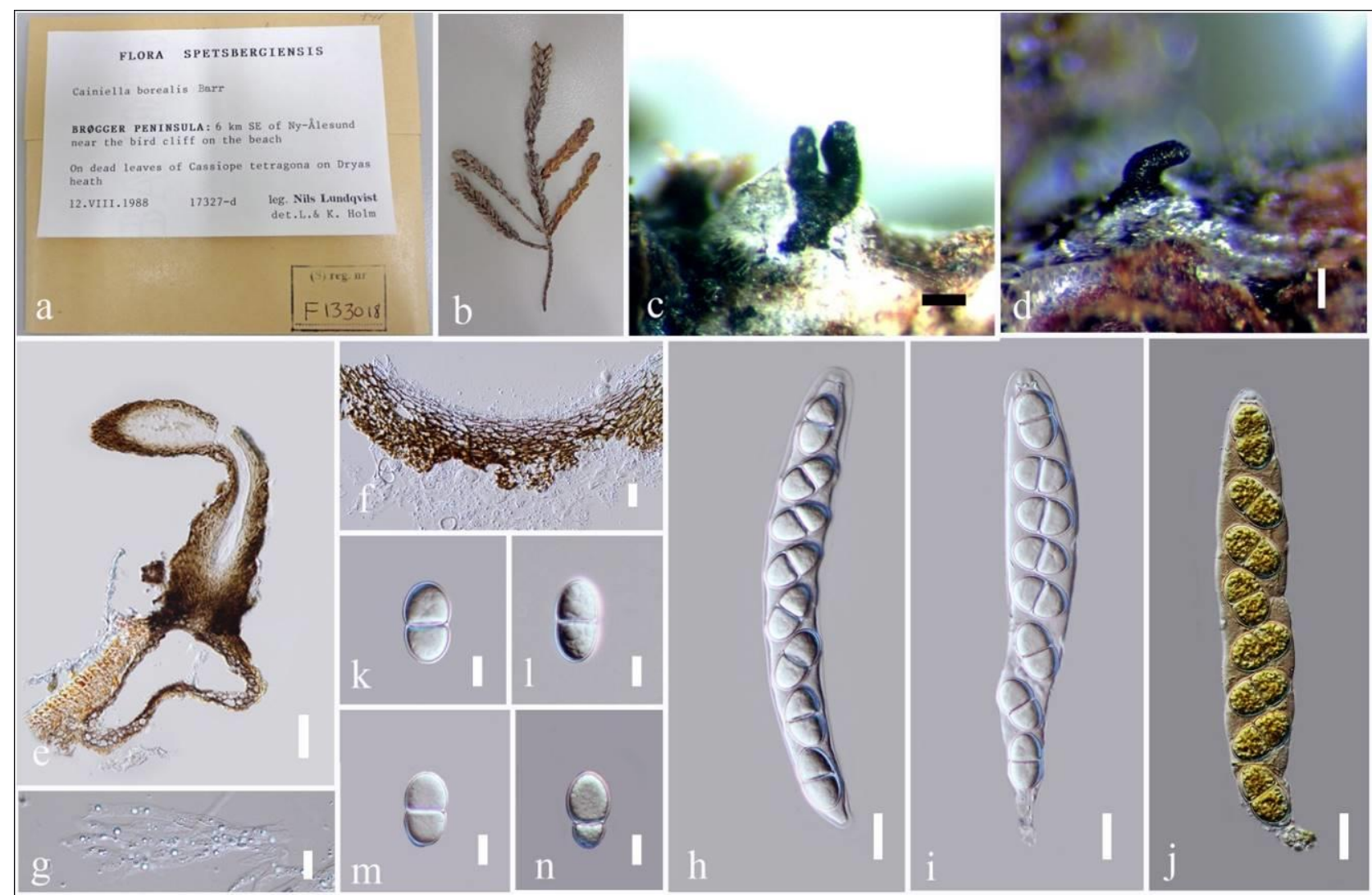

Figure 6 - Cainiella borealis (S-F133018). a Herbarium packet. b Herbarium specimen. c, d Appearance of ascomata on substrate. e Cross section of ascoma. f Peridium. g Paraphyses. h, i Asci in water. j Ascus in Melzer's reagent. k-n Ascospores. Scale bars $-\mathrm{c}=200 \mu \mathrm{m}, \mathrm{d}=200 \mu \mathrm{m}$, e $=100 \mu \mathrm{m}, \mathrm{f}, \mathrm{h}-\mathrm{j}=20 \mu \mathrm{m}, \mathrm{g}, \mathrm{k}-\mathrm{n}=10 \mu \mathrm{m}$.

Calosporella J. Schröt., in Cohn, Krypt.-Fl. Schlesien (Breslau) 3.2(4): 442 (1897) [1908] Facesoffungi number - FoF2841

Saprobic on dead branches of deciduous trees. Sexual morph - Pseudostromata pustulate on surface, circular, containing up to 20 ascoma. Ectostromatic disc erumpent, conspicuous, white to light grey, becoming dark at maturity, fusoid to circular, possessing 1-20 distinctly protruding ostioles. Entostroma crumbly, brown. Ascomata immersed in stromata, aggregated, globose to subglobose, coriaceous, black, ostiolate, papillate. Papilla erumpent, cylindrical, black. Asci 8spored, unitunicate, broadly fusoid, without or with inconspicuous pedicel, without a distinct apical ring. Ascospores bi- to triseriate, fusoid to oblong, 3-4-euseptate, hyaline, with tapering appendages at both ends. Asexual morph - Undetermined.

Type species - Calosporella innesii (Curr.) J. Schröt.

Calosporella innesii (Curr.) J. Schröt., in Cohn, Krypt.-Fl. Schlesien (Breslau) 3.2, 4: 442.1897 (1908).

Basionym - Sphaeria innesii Curr., Trans. Linn. Soc. London 22: 281. 1858 ('1859’).

Facesoffungi number - FoF2842

Epitype - WU 32161

Fig. 7

Saprobic on dead branches of deciduous trees. Sexual morph - Pseudostromata 1-3 mm diameter, pustulate on surface, circular, containing up to 20 ascomata. Ectostromatic disc erumpent, conspicuous, white to light grey, becoming dark at maturity, fusoid to circular, possessing 1-20 distinctly protruding ostioles. Entostroma crumbly, brownish. Ascomata 400-600 $\mu \mathrm{m}$ diameter, immersed in stromata, aggregated, globose to subglobose, coriaceous, black, ostiolate, papillate. 
Ostioles erumpent, cylindrical, black. Asci 60-100 × 16-22 $\mu \mathrm{m}$, 8-spored, unitunicate, broadly fusoid, without or with inconspicuous pedicel, lacking a distinct apical ring. Ascospores 20-34 $\times$ 6.5-10.5 $\mu \mathrm{m}$, bi- to triseriate, fusoid to oblong, 3-4-euseptate, not to slightly constricted at septa, hyaline, multi-guttulate, with rounded ends, hyaline tapering appendages at both ends, appendages 5-7 $\mu \mathrm{m}$ long, 3-4.5 $\mu \mathrm{m}$ wide at the base. Asexual morph - Undetermined (description based on Voglmayr \& Jaklitsch 2014).

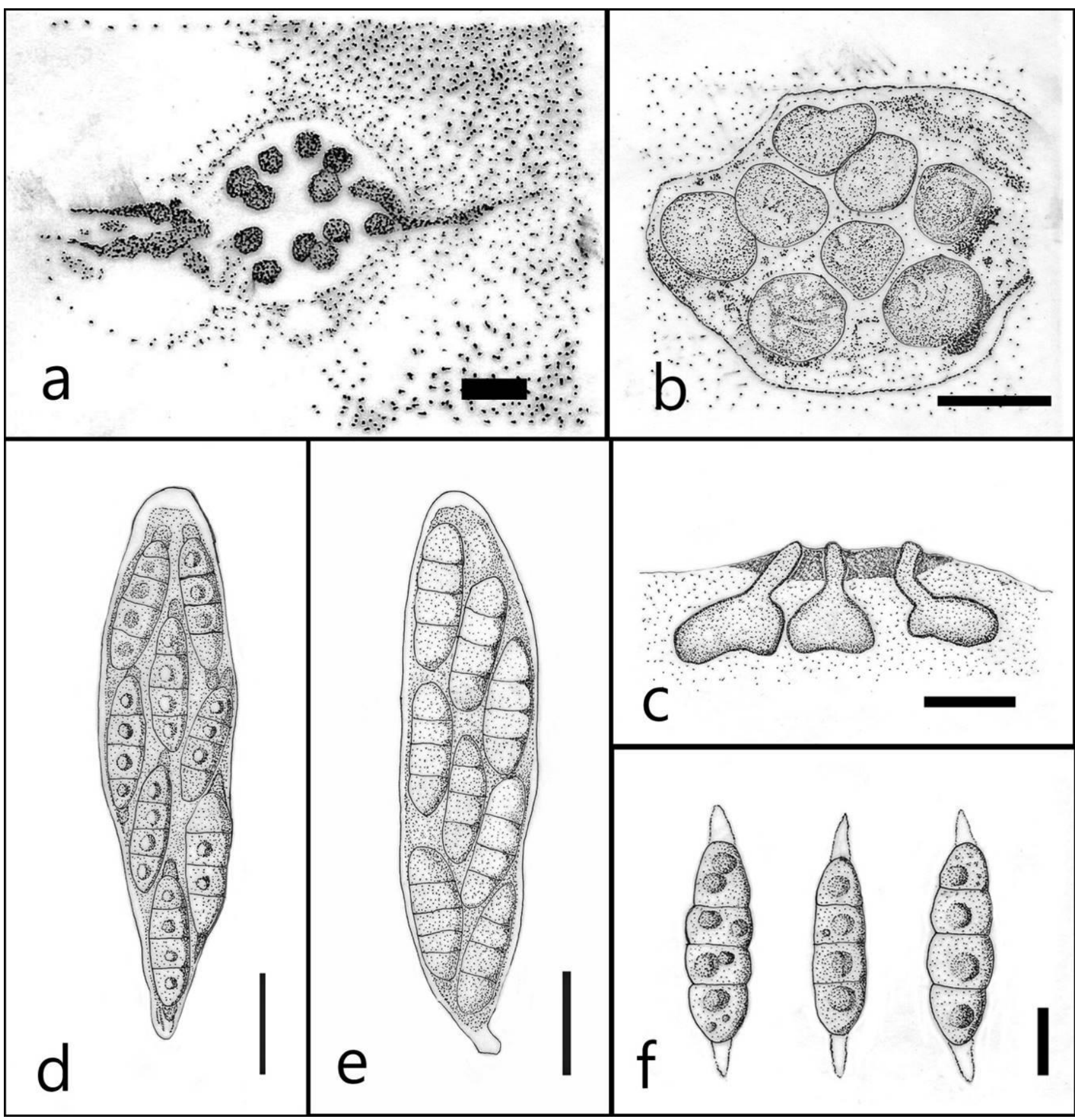

Figure 7 - Calosporella innesii. (Redrawn from Voglmayr \& Jaklitsch 2014). a Ascomata on substrate. b Horizontal cross section of ascoma. c Vertical cross section of ascoma. d, Matured ascus e. Over matured ascus. f Ascospores. Scale bars $-\mathrm{a}-\mathrm{c}=500 \mu \mathrm{m}, \mathrm{d}, \mathrm{e}=20 \mu \mathrm{m}, \mathrm{f}=10 \mu \mathrm{m}$.

Notes - Schröter (1897) erected the genus Calosporella as a replacement for Calospora Sacc. Saccardo (1883) listed 13 species under the genus without designating a generic type. However, the first two species, C. platanoidis (Pers.) Sacc. and C. innesii (Curr.) Sacc., are morphologically distinct (Saccardo 1883). Clements \& Shear (1931) lectotypified Calosporella with C. platanoidis. However, Wehmeyer (1941) suggested the type specimen of its basionym, Sphaeria platanoidis 
Pers., is not congeneric with $C$. platanoidis, based on its morphology. Hence lectotypification of the genus Calosporella, with $C$. platanoidis could not be accepted. Therefore, Voglmayr \& Jaklitsch (2014) typified Calosporella with $C$. innesii using a specimen collected from Austria associated with dead corticated branches of Acer pseudoplatanus. Calosporella innesii is characterized by hyaline, mostly 3-septate ascospores, with small apical appendages. It was transferred to Prosthecium by Wehmeyer (1941) based on phragmospores with apical appendages. However, in the prologue, Currey (1858) did not provide any details about the collection which was selected as the lectotype. Phylogenetic placement of Calosporella in Sydowiellaceae has been reported using ML analysis of the LSU sequence data from selected taxa in Diaporthales (Voglmayr \& Jaklitsch 2014).

Chapeckia M.E. Barr, Mycol. Mem. 7: 164 (1978)

Facesoffungi number - FoF2843

Saprobic on branches of deciduous trees and shrubs. Sexual morph - Stromata pseudoparenchymatous, scattered on host surface, immersed, opening to the outside through cracks in the host epidermis, appearing as an aggregation of ostioles, rounded or elliptic, externally black, internally brown, composed of compact pseudoparenchymatous tissues, 3-9 ascomata in a stroma. Ascomata upright, deeply immersed in stromata. Peridium thin at the base, wider at base of neck. Hamathecium comprising cellular paraphyses. Asci 8-spored, unitunicate, oblong to ellipsoidal, short-pedicellate. Ascospores biseriate, oblong-ellipsoidal to oval, light to dark brown, 1-median septate. Asexual morph - Undetermined.

Type species - Chapeckia nigrospora (Peck)

Chapeckia nigrospora (Peck) M.E. Barr, Mycol. Mem. 7: 165 (1978) [1880]

Basionym - Diatrype nigrospora Peck, Ann. Rep. N.Y. St. Mus. nat. Hist. 33: 33 (1883)

Facesoffungi number - FoF2844

Holotype - NYS 2083

Saprobic on branches of deciduous trees and shrubs. Sexual morph - Stromata 800-925 $\mu$ m wide $(\bar{x}=900 \mu \mathrm{m}, \mathrm{n}=10$ ), pseudoparenchymatous, scattered on host surface, immersed, opening to outside through cracks in the host epidermis, appearing as an aggregation of ostioles, rounded or elliptic in shape, externally black, internally brown, composed of compact pseudoparenchymatous tissues, 3-4 or up to 9 ascomata in a stroma. Ascomata 360-455 $\mu \mathrm{m}$ high, $160-225 \mu \mathrm{m}$ diameter $(\bar{x}$ $=408 \times 180 \mu \mathrm{m}, \mathrm{n}=20$ ), upright, deeply immersed in stromata, necks short and wide, flared at upper portion, globose. Peridium $10-13 \mu \mathrm{m}$ wide $(\bar{x}=11 \mu \mathrm{m}, \mathrm{n}=20)$, thin at the base, comprising 1-3 layers of brown-walled cells of textura angularis and base of neck comprising 6-8 layers of cells of textura angularis. Hamathecium comprising cellular paraphyses. Asci $40-55 \times 10-15 \mu \mathrm{m}$ $(\bar{x}=49 \times 12 \mu \mathrm{m}, \mathrm{n}=20), 8$-spored, unitunicate, oblong to ellipsoidal, apex slightly pointed, shortpedicellate. Ascospores 9.5-11 ×5-6 $\mu \mathrm{m}(\bar{x}=10.7 \times 5.6 \mu \mathrm{m}, \mathrm{n}=20)$, biseriate, oblong-ellipsoidal to oval, hyaline when immature, light to dark brown at maturity, 1-median septate, slightly constricted at the septum, wall surrounded by narrow gel coating becoming irregularly rough when dry, appendages narrow, short or elongate. Asexual morph - Undetermined.

Material examined - USA, New York, Quaker Street, on bark of Betula lutea, C.H. Peck (NYS 2083, holotype).

Notes - Chapeckia was introduced by Barr (1978) based on Diatrype nigrospora and accommodated in Pseudovalsaceae. There are two species in the genus (Index Fungorum 2017) the type species and C. ribesia (Sacc. \& Scalia) M.E. Barr.

Lumbsch \& Huhndorf (2007) placed Chapeckia in Melanconidaceae while Rossman et al. (2007) placed it in Sydowiellaceae. Later, Lumbsch \& Huhndorf (2010) also accommodated Chapeckia in Sydowiellaceae. De Silva et al. (2009) and Kruys \& Castlebury (2012) analyzed sequence data for Chapeckia and agreed it belonged in Sydowiellaceae. 


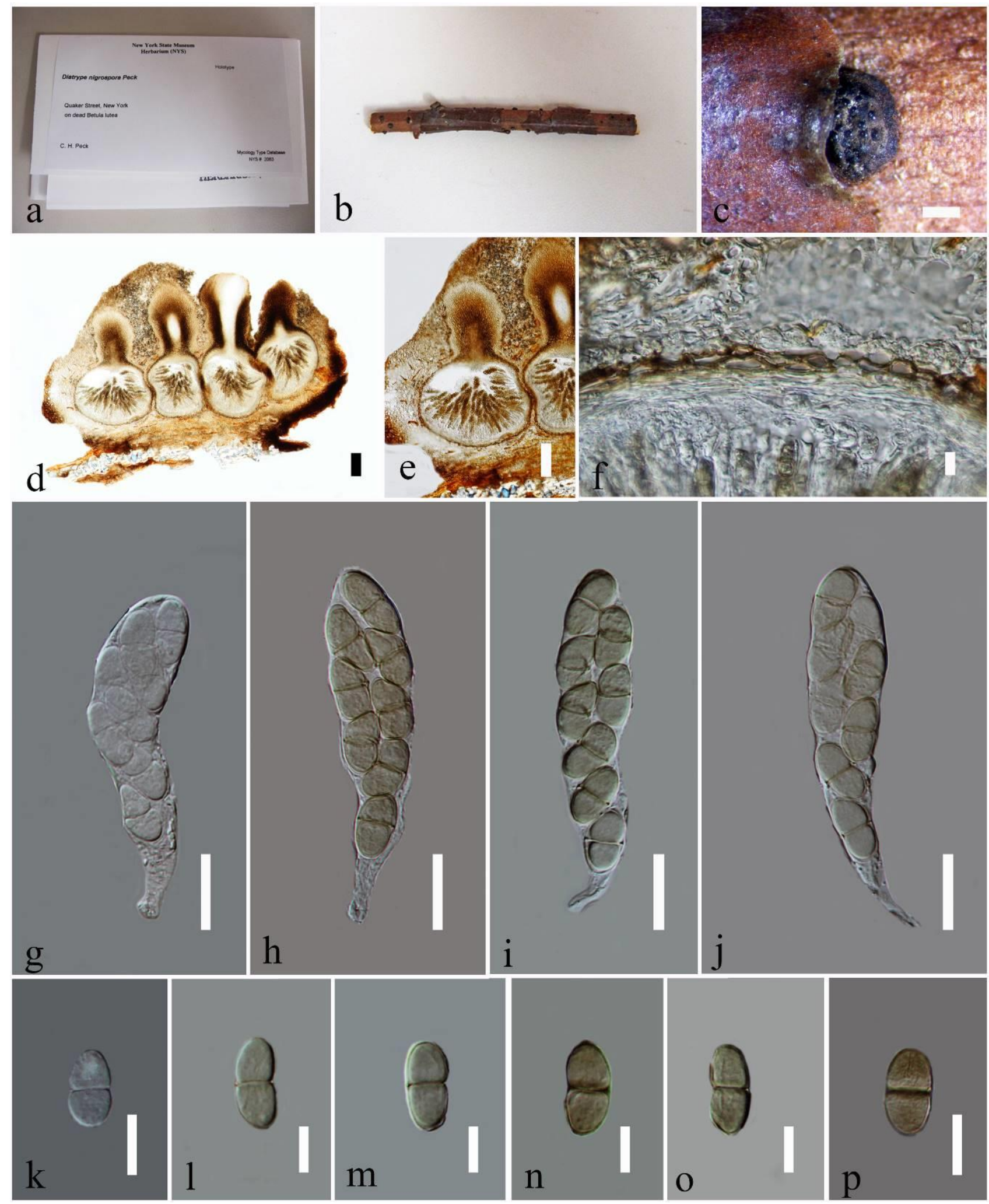

Figure 8 - Chapeckia nigrospora (holotype). a, b Herbarium packet and specimen. c Appearance of stroma on host surface. d-e Cross section of ascomata in stroma. f Peridium. g-j Asci. k-p Ascospores. Scale bars $-\mathrm{c}=500 \mu \mathrm{m}, \mathrm{d}, \mathrm{e}=100 \mu \mathrm{m}, \mathrm{f}=10 \mu \mathrm{m}, \mathrm{g}-\mathrm{j}=20 \mu \mathrm{m}, \mathrm{k}-\mathrm{p}=10 \mu \mathrm{m}$.

Hapalocystis Auersw. ex Fuckel, Fungi rhenani exsic., fasc. 6: no. 585 (1863)

Facesoffungi number - FoF2845

Saprobic on woody bark. Sexual morph - Stromata forming a thin, weft of light brown hyphae around upper part of ascomata. Ascomata immersed, aggregated, subglobose to globose, coriaceous, black, ostiolate, papillate. Papilla short, black, cylindrical, periphysate. Peridium 
comprising few layers of black, thick-walled, cells of textura angularis. Hamathecium comprising a few, septate, hyaline paraphyses attached to the base, longer than asci. Asci 4 or 8-spored, unitunicate, clavate to fusoid, with a short pedicel, and an inconspicuous flat, J-, refractive ring. Ascospores inequilaterally ellipsoidal, with broadly rounded ends, hyaline to dark brown, distoseptate, with short or long, hyaline strap-like appendages at both rounded ends. Asexual morph: Coelomycetous. Conidiomata pycnidia, superficial, aggregated in groups of 3-5, globose, orange to brown. Conidiomatal wall small, thick-walled, orange, comprising cells of textura angularis. Conidiophores annelidic, branched, hyaline, short, few conidiogenous cells arising from a single conidiophore, attached to conidiomatal wall. Conidiogenous cells cylindrical, hyaline, bottle-shaped, septate, ends pointed, phialidic. Conidia ellipsoid or cylindroid, unicellular or multiseptate, hyaline or brown.

Type species - Hapalocystis berkeleyi Auersw. ex Fuckel, Fungi rhenani exsic., fasc. 6: no. $585(1863)$

Hapalocystis berkeleyi Auersw. ex Fuckel, Fungi rhenani exsic., fasc. 6: no. 585 (1863)

Facesoffungi number - FoF2846

Isotype - K(M) 195743

Saprobic on woody bark. Sexual morph - visible through dark cracks on the host surface. Stromata forming a thin, weft of light brown hyphae around upper part of ascomata. Ascomata 90-185 $\mu \mathrm{m}$ high, 206-302 $\mu \mathrm{m}$ diameter $(\bar{x}=150 \times 245 \mu \mathrm{m}, \mathrm{n}=15)$, immersed, aggregated, subglobose to globose, coriaceous, black, ostiolate. Ostiole $130-195 \mu \mathrm{m}$ high, $90-113 \mu \mathrm{m}$ wide $(\bar{x}=145 \times 105$ $\mu \mathrm{m}, \mathrm{n}=10$ ), short papillate, black, cylindrical, periphysate with hyaline, filamentous periphyses. Peridium 4-16 $\mu \mathrm{m}$ wide $(\bar{x}=10 \mu \mathrm{m}, \mathrm{n}=15)$, comprising 3-5-layers of black, thick-walled cells of textura angularis. Paraphyses 3-7 $\mu \mathrm{m}$ wide $(\bar{x}=6 \mu \mathrm{m}, \mathrm{n}=15)$, few, septate, hyaline, attached to the base, longer than asci. Asci 112-127 $\times 46-80 \mu \mathrm{m}(\bar{x}=121 \times 66 \mu \mathrm{m}, \mathrm{n}=20), 4$-or 8-spored, unitunicate, clavate to fusoid, with a short pedicel, with an inconspicuous flat, refractive ring at the lower end of the thickened apical wall, apex narrow and blunted. Ascospores 34-42 $\times 12-18 \mu \mathrm{m}$ $(\bar{x}=37 \times 17 \mu \mathrm{m}), 2-5$-seriate, inequilaterally ellipsoidal, with broadly rounded ends, initially hyaline, dark brown at maturity, (1-)-2-(-3)-septate, with cells of equal length, thick and smoothwalled, with short, hyaline, strap-like appendages situated at both rounded ends, having the same width as the ascospores. Asexual morph - Coelomycetous. Conidiomata 165-195 $\mu \mathrm{m}$ diameter $(\bar{x}$ $=182 \mu \mathrm{m}, \mathrm{n}=15$ ), pycnidial, superficial, aggregated, 3-5 in a group, globose, orange to brown. Conidiomata wall $7-13 \mu \mathrm{m}$ wide $(\bar{x}=10 \mu \mathrm{m}, \mathrm{n}=10)$, comprising 5-10 layers of small, thickwalled, orange cells of textura angularis. Conidiophores $1.5-2 \mu \mathrm{m}$ high, $1-1.5 \mu \mathrm{m}$ wide $(\bar{x}=2 \times$ $1.5 \mu \mathrm{m}, \mathrm{n}=10$ ), branched, hyaline, short, few conidiogenous cells arising from a single conidiophore, attached to conidiomatal wall. Conidiogenous cells 10-14 $\mu \mathrm{m}$ high, 5-7 $\mu \mathrm{m}$ wide, cylindrical, hyaline, bottle-shaped, septate, pointed at ends, phialidic. Conidia $0.5-1.5 \mu \mathrm{m}$ diameter $(\bar{x}=1 \mu \mathrm{m}, \mathrm{n}=20)$ ellipsoid, 1-celled, hyaline, smooth-walled.

Material examined - ITALY, Province of Forlì-Cesena [FC], Modigliana, Montebello, on branch of Platanus hybrida L. (Platanaceae), 14 April 2013, Erio Camporesi, IT 1187 (MFLU 140798).

Notes - Hapalocystis was established to accommodate two species, $H$. berkeleyi and $H$. bicaudata Fuckel (Fuckel 1863). Clements \& Shear (1931) designated H. berkeleyi as the type species. Later this genus was synonymized under Pseudovalsa (Winter 1887, Ellis \& Everhart 1892) and Prosthecium (Wehmeyer 1941).

Barr (1978) however excluded Hapalocystis from Prosthecium based on morphology and placed the genus in Melanconidaceae (Barr 1978, Lumbsch \& Huhndorf 2007). Rossman et al. (2007) placed Hapalocystis in Sydowiellaceae based on its morphology. Hapalocystis now comprises H. bicaudata H. corni (Wehm.) M.E. Barr, H. corticalis (Schwein.) M.E. Barr, $H$. mirabilis Sorokīn, H. occidentalis Jaklitsch \& Voglmayr, H. ulmi (Wehm.) M.E. Barr, and $H$. berkeleyi ( $H$. berkeleyi var. berkeleyi and $H$. berkeleyi var. kickxii (Westend.) M.E. Barr and $H$. vexans Speg. (Index Fungorum 2017). The asexual morph of Hapalocystis was recorded as 
Stilbospora, Hendersonia or Dothiorella (Jaklitsch \& Voglmayr 2004). Glawe (1985) and Liu et al. (2015) reported a Phoma-like asexual morph for $H$. berkeleyi from culture.
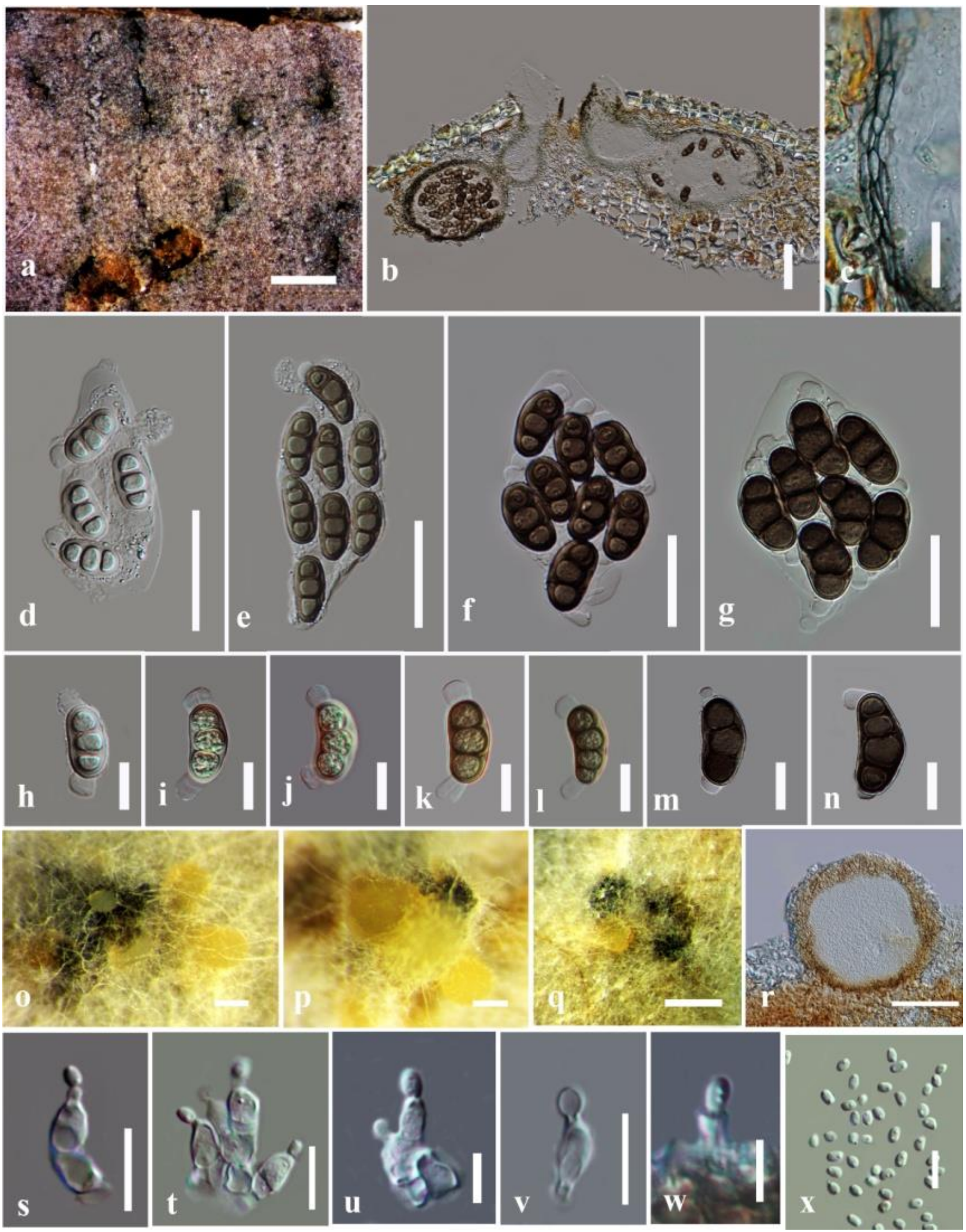

Figure 9 - Hapalocystis berkeleyi. a Appearance of ascomata on substrate. b Vertical cross section of ascomata. c Peridium. d-g Asci. $\mathrm{h}-\mathrm{n}$ Ascospores. o-q Conidiomata. $\mathrm{r}$ Vertical cross section of conidioma. s-w Conidiophores, conidiogenous cells and conidia. $\mathrm{x}$ Conidia. Scale bars $-\mathrm{a}=1 \mathrm{~mm}$, $\mathrm{b}=100 \mu \mathrm{m}, \mathrm{c}=20 \mu \mathrm{m}, \mathrm{d}-\mathrm{g}=50 \mu \mathrm{m}, \mathrm{h}-\mathrm{n}=20 \mu \mathrm{m}, \mathrm{o}-\mathrm{q}=500 \mu \mathrm{m}, \mathrm{g}=100 \mu \mathrm{m}, \mathrm{s}-\mathrm{w}=10 \mu \mathrm{m}, \mathrm{x}=5$ $\mu \mathrm{m}$. 
Italiomyces Senan., Camporesi \& K.D. Hyde, gen. nov.

Index Fungorum number - IF552721, Facesoffungi number - FoF 2847

Etymology - Based on the country where the fungus was collected.

Saprobic on dead stem of Centaurea L. Sexual morph - Ascomata solitary to rarely aggregated, scattered, immersed, globose to subglobose, black coriaceous, papillate, ostiolate. Papilla short, wide, emerging through cracks in the substrate, comprising small, thick-walled, cells of textura angularis, internally covered by hyaline, periphyses. Peridium comprising thick-walled, compressed, dark brown cells of textura angularis. Asci 8-spored, unitunicate, fusiform, shortpedicellate, apex blunt with bi-lobed apical ring. Ascospores overlapping uniseriate to biseriate, fusiform to narrowly oval, 1-septate, large, hyaline, with appendages. Asexual morph Undetermined.

Type species - Italiomyces centaureii Senan., Camporesi \& K.D. Hyde

Italiomyces centaureii Senan., Camporesi \& K.D. Hyde, sp. nov.

Index Fungorum number - IF552722, Facesoffungi number - FoF $2848 \quad$ Fig. 10

Etymology - Based on the host genus.

Holotype - MFLU 16-2866

Saprobic on dead stems of Centaurea L. Sexual morph - Ascomata 360-380 $\mu \mathrm{m}$ high, 405-415 $\mu \mathrm{m}$ diameter $(\bar{x}=375 \times 411 \mu \mathrm{m}, \mathrm{n}=15)$, solitary to rarely aggregated, scattered, immersed, globose to subglobose, black, coriaceous, papillate, ostiolate. Papilla 140-165 $\mu \mathrm{m}$ high, $150-185 \mu \mathrm{m}$ diameter $(\bar{x}=160 \times 175 \mu \mathrm{m}, \mathrm{n}=15)$, emerging through cracks in the substrate, comprising small, thickwalled cells of textura angularis, internally covered by hyaline, periphyses. Peridium 15-25 $\mu \mathrm{m}$ wide $(\bar{x}=20 \mu \mathrm{m}, \mathrm{n}=15)$, comprising thick-walled, compressed, dark brown cells of textura angularis. Asci $90-110 \times 15-20 \mu \mathrm{m}(\bar{x}=105 \times 17 \mu \mathrm{m}, \mathrm{n}=25)$, 8-spored, unitunicate, fusiform, short-pedicellate, apex blunt with bi-lobed apical ring. Ascospores 20-30 × 6.5-8.5 $\mu \mathrm{m}(\bar{x}=26 \times$ $7.7 \mu \mathrm{m}, \mathrm{n}=25$ ), overlapping uniseriate to biseriate, fusiform to narrowly oval, 1-septate, biguttulate, hyaline, with short, spine-like appendages at both ends. Asexual morph - Undetermined.

Culture characters - Colonies growing on MEA attaining $1 \mathrm{~cm}$ within 7 days when incubated at $18^{\circ} \mathrm{C}$, with sparse aerial mycelia, forming minute mycelia tufts, powdery, flat, circular, smooth, white to off-white.

Material examined - ITALY, Province of Forlì-Cesena, Fiumicello di Premilcuore, on dead stem of Centaurea sp. (Asteraceae), 2 April 2014, Erio Camporesi, IT 1976 (MFLU 16-2866, holotype), ex-type living cultures MFLUCC 14-0849, ibid (BBH isotype), ex-type living culture, TBRC.

Notes - Italiomyces is distinct from other genera in Sydowiellaceae in its bi-guttulate or rarely 4guttulate ascospores with minute, spine-like apical appendages. The guttule arrangement is unique for this genus and comprising two guttules located very close to each septum and smaller guttules at the ends of the spores. Furthermore, phylogenetically Italiomyces is distinct (Fig. 1) and hence we introduce the new genus.

Lambro Racib., Parasit. Alg. Pilze Java's (Jakarta) 2: 13 (1900)

= Stegophora Syd. \& P. Syd., Annls mycol. 14(5): 364 (1916)

Index Fungorum number - IF552801 Facesoffungi number - FoF 2849

Pathogenic forming spots on living leaves. Sexual morph - Stromata pseudoparenchymatous to prosenchymatous, well-developed, black, with 3-12 ascomata in a stroma, forming a dark outer crust around the hyaline interior. Ascomata solitary, scattered, vertically or obliquely immersed within leaf tissue, globose to subglobose, coriaceous, black, with short neck and effuse clypeus-like hypostroma. Peridium membranaceous, outer layer comprising brown, thick-walled cells of textura angularis and inner layer of hyaline, thin-walled cells of textura angularis. Necks central or asymmetrically located, slightly protruding from either upper or lower leaf surface as blackish papillae, broader near the tip, internally covered by hyaline periphyses, wall comprising plectenchymatous, vertically elongate cells. Hamathecium aparaphysate. Asci 8-spored, 


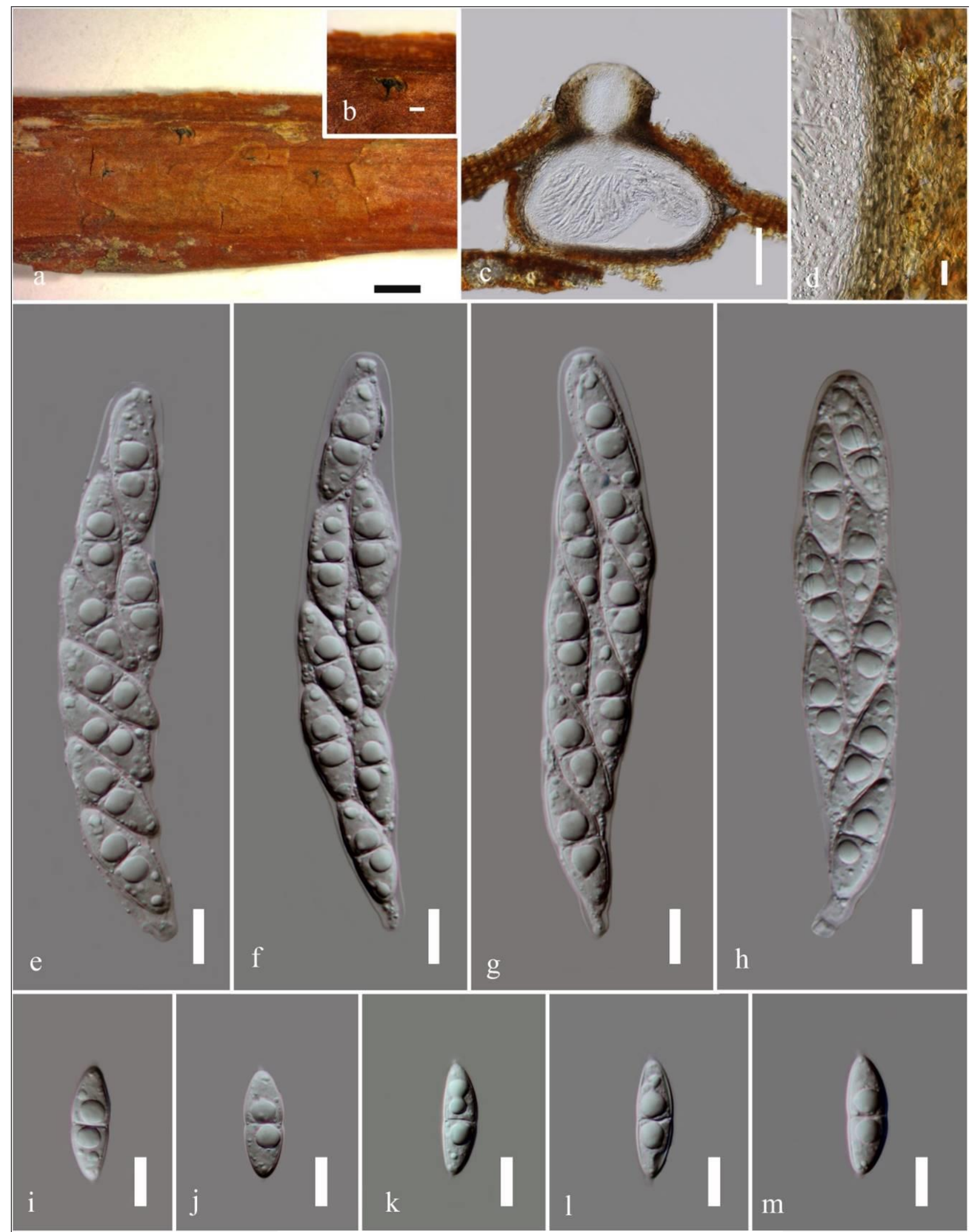

Figure 10 - Italiomyces centaureii (holotype). a, b Herbarium specimen. c Vertical section of ascoma. d Peridium. e-h Asci. i-m Ascospores. Scale bars: $a=1 \mathrm{~mm}, \mathrm{~b}=200 \mu \mathrm{m}, \mathrm{c}=100 \mu \mathrm{m}, \mathrm{d}-\mathrm{m}$ $=10 \mu \mathrm{m}$.

unitunicate, clavate, tapering towards the base, apex thickened with $\mathrm{J}$ - apical ring. Ascospores overlapping uniseriate, apiosporous, elliptic to ovoid, tapering towards the base, hyaline. Asexual morph - Coelomycetous, Cylindrosporella-like. Appearing as greyish-brown to brown, circular or 
irregularly-shaped. Conidiomata acervuli, scattered, solitary, erumpent. Conidiophores simple, short or obscure. Conidiogenous cells phialidic. Conidia hyaline, small, unicellular, filiform, fusiform, clavate or fusoid.

Type species - Lambro insignis Racib., Parasit. Alg. Pilze Java's (Jakarta) 2: 10 (1900)

Lambro oharana (Y. Nisik. \& H. Matsumoto) E. Müll., in Müller \& von Arx, Beitr. Kryptfl. Schweiz 11(no. 2): 778 (1962)

Facesoffungi number - FoF 2850

Fig. 11

Parasitic forming yellowish spots on living leaves, without distinct marginal zone, aggregated in a small black phyllachoroid scab, which is the cover of the conidial layer. Sexual morph - Stromata hypostromatic, pseudoparenchymatous, spread beneath ascomata, surrounded by clypeus in epidermal layer. Ascomata 370-480 $\mu \mathrm{m}$ diameter, entirely immersed within leaf tissue, usually located as pairs, brown to light brown, globose to subglobose, with central or asymmetrically located short neck. Necks 120-180 $\mu \mathrm{m}$ high, 100-110 $\mu \mathrm{m}$ wide, slightly elevated from the upper leaf surface, parallel between paired ascomata, broader near the apex, with hyaline periphyses. Peridium plectenchymatous, light brown. Asci $43-56 \times 7.5-10 \mu \mathrm{m}(\bar{x}=48.8 \times 8.8 \mu \mathrm{m})$, 8-spored, clavate, tapering toward the base, thin-walled, with slightly visible apical ring at the thickened apex, irregularly loose in ascomata. Ascospores $12.5-15 \times 5-6.5 \mu \mathrm{m}(\bar{x}=13.4 \times 5.5 \mu \mathrm{m})$, irregularly biseriate, apiosporous, elliptic to ovoid, hyaline. Asexual morph - Coelomycetous. Conidiomata $0.45-0.5 \mathrm{~mm}$ diameter, acervuli, subepidermal, initially with thick, black cover, flat, round to elliptic. Conidiophores obscure. Conidiogenous cells phialidic. Conidia 4.5-8 $\times 1.5-2.5$ $\mu \mathrm{m}(\bar{x}=5.4 \times 1.5 \mu \mathrm{m})$, clavate or oblong-ovoid, straight or curved, hyaline, unicellular (description is based on Kobayashi 1970).

Notes - The genus Lambro is typified by L. insignis, which produces necrotic spots in leaves of Sterculia subpeltata Blume (Sterculiaceae) (Raciborski 1900). Müller \& von Arx (1962) described and illustrated this genus and reported the morphological similarity with Stegophora and Metadothella Henn. species. Lambro insignis has a well-developed pseudoparenchymatous stromata completely enveloping 3-12 ascomata, and a dark outer crust around the hyaline interior region. However, the type species of Stegophora, S. ulmea has only subepidermal hypostroma (Müller \& von Arx 1962). Stegophora oharana forms both a clypeus and hypostroma, which is limited to the epidermal layer of leaves. Sydow (1936) described S. aenula Syd. on Ulmus davidiana collected in China. Morphologically and biologically this species is identical to $S$. oharana. Stegophora oharana was transferred to Lambro based on its morphological similarity. Monod (1983) examined the type species, L. insignis and suggested that this species is related to Stegophora, thus it may belong in Sydowiellaceae. Hence, we synonymize Stegophora under Lambro giving priority to the older generic name Lambro and ignoring the small morphological differences between stromata. The asexual morph of Lambro is reported as a Cylindrosporella-like species.

Paragnomonia Senan. \& K.D. Hyde, gen. nov.

Index Fungorum number - IF552723, Facesoffungi number - FoF 2851

Etymology - In reference to the similarity to Gnomonia.

Pathogenic on plants. Sexual morph - Ascomata immersed, on roots and petioles, only neck visible when associated with host, solitary, globose to subglobose, black, coriaceous, papillate, ostiolate. Papilla central, straight to curved. Peridium comprising outer layer of large, brown cells of textura angularis and inner layer of compressed, hyaline cells of textura angularis. Asci fusiform to obovoid, with short pedicel and J- apical ring. Ascospores obliquely uniseriate, fusiform, straight to curved, 1-median-septate, not constricted at septum, with 4-6 guttules in each cell, sometimes more than 6-guttulate. Appendages apical and basal, hyaline. Asexual morph Conidiomata tiny, pycnidia, solitary, immersed to erumpent, black. Conidiophores reduced to conidiogenous cells. Conidiogenous cells elongate, conical, unbranched, hyaline. Conidia oval to ellipsoid, aseptate, 2-guttulate, hyaline (description based on Alexopoulos \& Cation 1952). 
Type species - Paragnomonia fragariae (Kleb.) Senan. \& K.D. Hyde

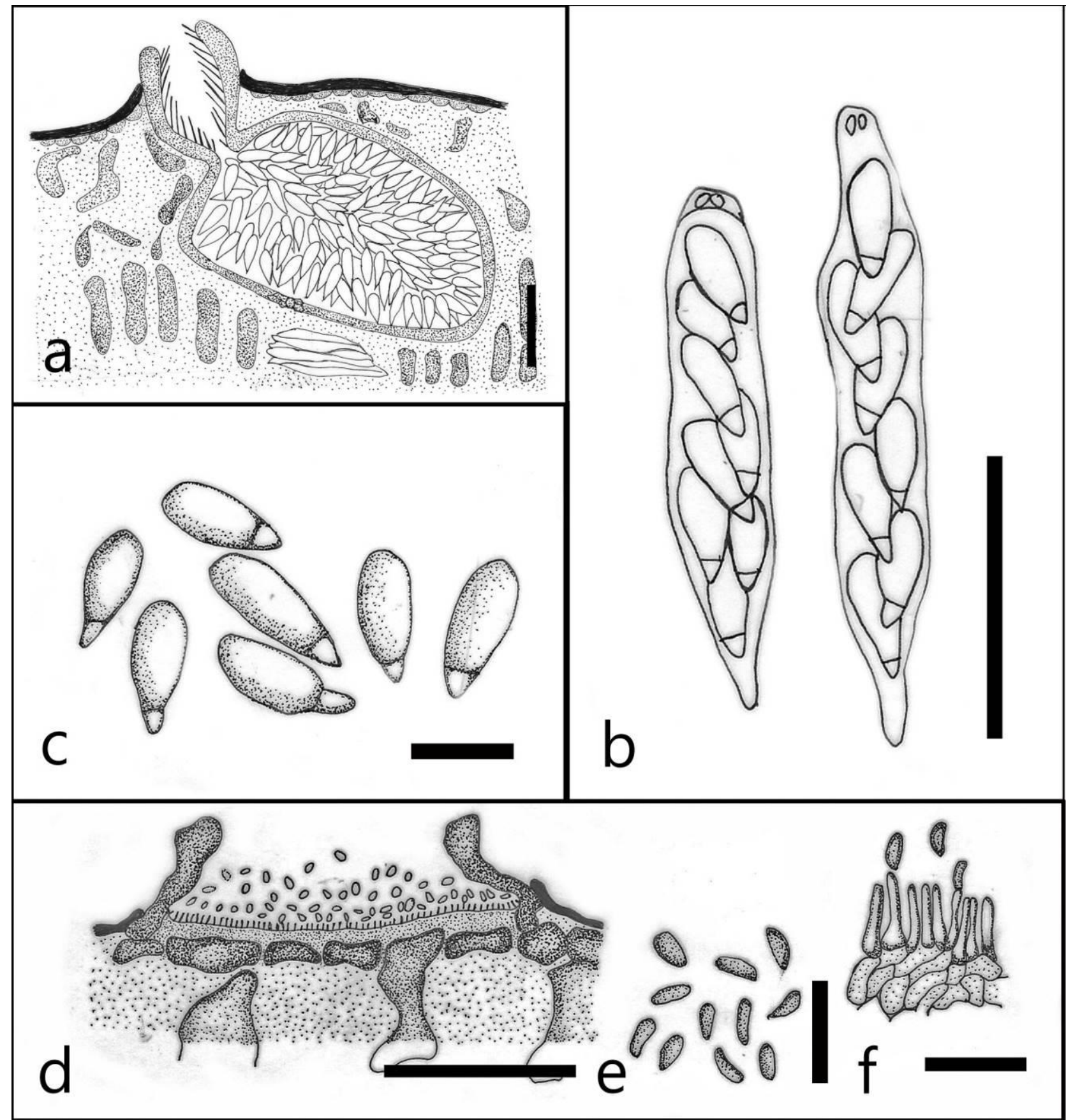

Figure 11 - Lambro oharana (drawing based on Kobayashi 1970) a Vertical section of ascomata. b Asci. c Ascospores. d Vertical section of conidiomata. e Conidia. $\mathrm{f}$ Conidiogenous cells attached to conidia. Scale bars $-\mathrm{a}, \mathrm{d}=100 \mu \mathrm{m}$. b, c, e, $\mathrm{f}=10 \mu \mathrm{m}$.

Paragnomonia fragariae (Kleb.) Senan. \& K.D. Hyde, comb. nov.

Index Fungorum number - IF552724, Facesoffungi number - FoF 2852

Figs 12, 13

= Gnomonia fragariae var. fragariae Kleb., Haupt- und Nebenfruchtformen der Ascomyzeten (Leipzig) 1: 285 (1918)

Pathogenic on plants. Sexual morph - Ascomata immersed on roots and petioles, papilla only visible on host, solitary, globose to subglobose, black, coriaceous, papillate, ostiolate. Necks central, straight to curved. Peridium comprising outer layer of large, brown cells of textura angularis and inner layer of compressed, hyaline cells of textura angularis. Asci 25-35 × 6-7 $\mu \mathrm{m}$, fusiform to obovoid, with short pedicel and J- apical ring. Ascospores 8-11 $\times 2-4 \mu \mathrm{m}$, obliquely uniseriate, fusiform, straight to curved, 1-median septate, not constricted at the septum, with 4-6 




Figure 12 - Paragnomonia fragariae (drawing based on Morocko \& Fatehi 2007). a Ascospores on substrate. b Vertical cross section of ascomata. c Asci. d Ascospores. Scale bars: $\mathrm{c}, \mathrm{d}=10 \mu \mathrm{m}$.

guttules, sometimes more than 6-guttulate. Appendages apical and basal, hyaline, elongate-conical. Asexual morph - Conidiomata tiny, pycnidial, solitary, immersed to erumpent, black. Conidiophores reduced to conidiogenous cells. Conidiogenous cells elongated, conical, unbranched, hyaline. Conidia oval to ellipsoid, aseptate, bi-guttulate, hyaline (description based on Alexopoulos \& Cation 1952).

Notes - Paragnomonia fragariae is a poorly studied taxon, which was described by Klebahn (1918) from dead petioles and leaves of strawberry and causes strawberry decline in Germany. Klebahn (1918) considered this fungus as a saprobe of dead tissues of strawberry. Later, $P$. fragariae was recorded on dead tissues of pink barren strawberry (Potentilla micrantha), silver weed or goose grass ( $P$. anserina), rock cinquefoil ( $P$. rupestris), woodland strawberry (Fragaria vesca) and cultivated strawberry (Fragaria xananassa) collected in several localities of Switzerland 


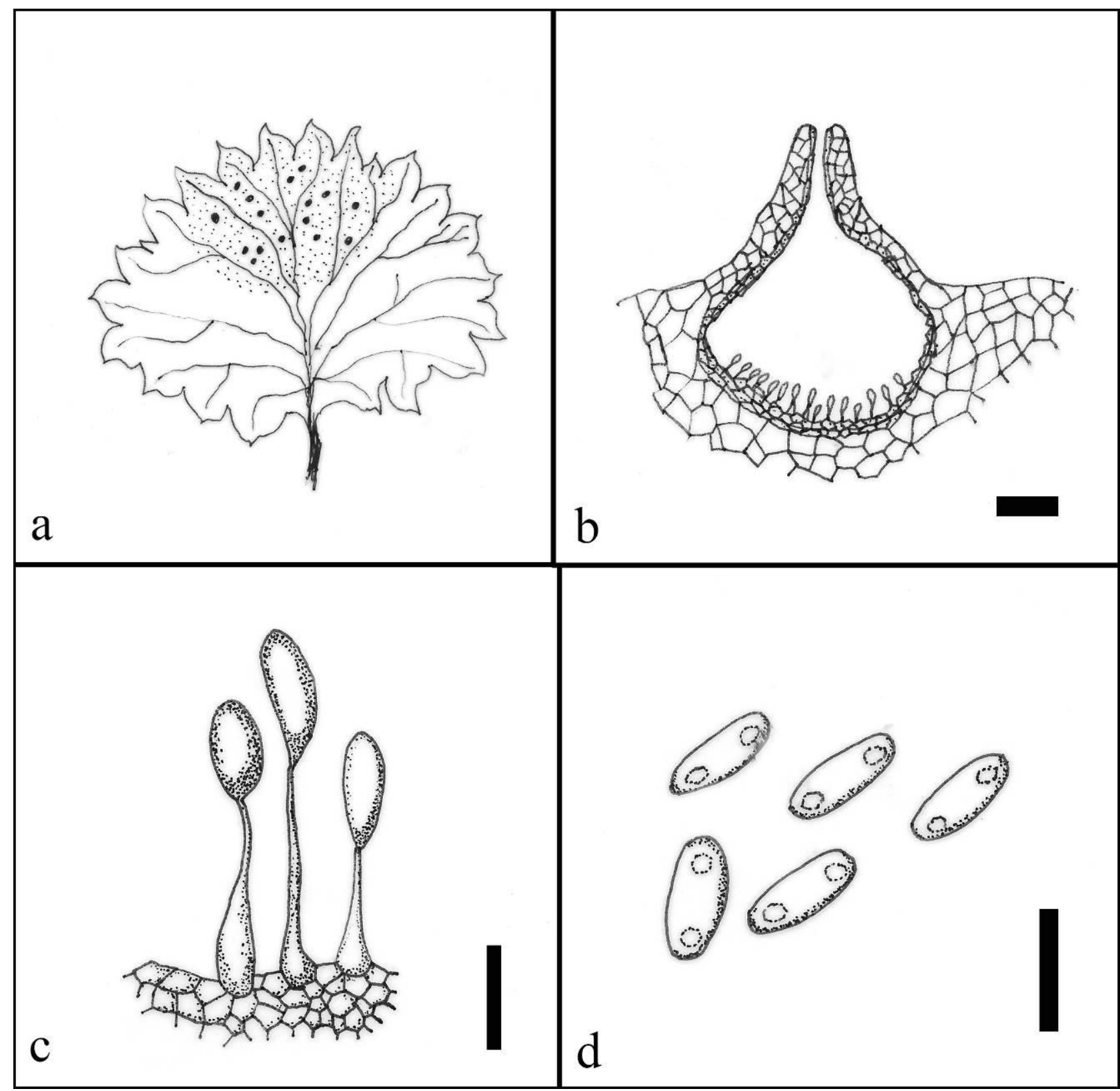

Figure 13 - Paragnomonia fragariae (drawing based on Alexopoulos \& Cation 1952) a Conidiomata on host leaf. b Cross section of conidioma. c Conidiophore, conidiogenous cells attached to conidia. $\mathrm{d}$ Conidia. Scale bars $-\mathrm{b}=100 \mu \mathrm{m}, \mathrm{c}-\mathrm{d}=5 \mu \mathrm{m}$.

(Bolay 1972). A severe strawberry petiole blight caused by Gnomonia comari was reported by van Adriechem and Bosher (1958) and is very similar to petiole blight caused by P. fragariae. However, Gnomonia comari did not cause any disease symptoms on roots, unlike $P$. fragariae. Morocko (2006) described the ascomata, asci and ascospores from cultures grown on artificial media and naturally infected plant tissues, with similar characteristics to $P$. fragariae (Klebahn 1918) and herbarium specimens collected by Bolay (1972) and Monod (1983). Morocko (2006) noted a great variation in length of the ascospore appendages.

Farr et al. (1989) and Maas (1998) suggested P. fragariae was a synonym of Gnomonia comari and Sogonov et al. (2008) placed G. comari in the newly introduced genus Gnomoniopsis. Gnomoniopsis comari has smaller asci and ascospores with a submedian septum and lacks appendages (Barr 1978). Morphologically Gnomoniopsis comari shows similarity to only Gnomonia fragariae var. fructicola (G. Arnaud) Fallahyan and not to Gnomonia fragariae var. fragariae Kleb. Morocko (2006) analyzed LSU and ITS sequence data of Gnomonia species and members of Diaporthales and showed that G. fragariae var. fragariae is phylogenetically 
segregated from the generic type G. gnomon, and most other members of Gnomoniaceae. Morocko (2006) also suggested that G. fragariae f. fragariae, G. rubi, G. rosae and Hapalocystis species represents a genetically distinct group, possibly a new family, within Diaporthales.

Combined gene analyses of LSU, ITS, RPB2 and TEF sequence data in this study confirm the phylogenetic placement of Gnomonia fragariae var. fragariae in Sydowiellaceae. Hence, we exclude Gnomonia fragariae var. fragariae from Gnomoniopsis comari and introduce a new genus, Paragnomonia to accommodate Gnomonia fragariae var. fragariae.

Ranulospora Senan., Camporesi \& K.D. Hyde, gen. nov.

Index Fungorum number - IF552725, Facesoffungi number - FoF 2853

Etymology - Name referring to the tadpole-like shape of ascospores.

Saprobic on dead branches of deciduous plants. Sexual morph - Appearing as clusters of black, ostiolate necks which are erumpent. Pseudostromata comprising sparse, brown, fungal hyphae spreading around the ascomata. Ascomata immersed, aggregated, globose to subglobose, coriaceous, dark brown to black, with 6-8 aggregated ascomata, with long ostiolar necks. Peridium composed of 4-6 outer layers of thick-walled, brown cells of textura angularis and inner, hyaline, thick-walled cells of textura angularis. Hamathecium comprising few, septate, thin-walled paraphyses, which are longer than the asci. Asci 8-spored, unitunicate, cylindrical to fusiform, short pedicellate, apex rounded, with $\mathrm{J}$ - apical ring. Ascospores uniseriate or biseriate, fusiform to ellipsoid, with long basal appendage. Asexual morph - Undetermined.

Type species - Ranulospora alnii Senan., Camporesi \& K.D. Hyde

Ranulospora alnii Senan., Camporesi \& K.D. Hyde, sp. nov.

Index Fungorum number - IF552726, Facesoffungi number - FoF $2854 \quad$ Fig. 14

Etymology - Named after the host genus.

Holotype - MFLU 16-2868

Saprobic on dead branches of Alnus incana (L.) Moench. Sexual morph - Appearing as clusters of black, ostioles emerging through the cracked bark. Pseudostromata comprising sparse, brown, fungal hyphae spreading around ascomata. Ascomata 480-765 $\mu \mathrm{m}$ high, 280-345 $\mu \mathrm{m}$ diameter $(\bar{x}=640 \times 315 \mu \mathrm{m}, \mathrm{n}=20)$, immersed, aggregated, globose to subglobose, coriaceous, dark brown to black, with 6-8 ascomata in an aggregation, papillate, with long ostiolar necks. Papilla 320-380 $\mu \mathrm{m}$ high, $160-260 \mu \mathrm{m}$ wide $(\bar{x}=349 \times 207 \mu \mathrm{m}, \mathrm{n}=20)$. Peridium $13-23 \mu \mathrm{m}$ wide $(\bar{x}=16.8 \mu \mathrm{m}, \mathrm{n}=20)$ composed of $4-6$ outer layers of thick-walled, brown cells of textura angularis and inner, hyaline, thick-walled cells of textura angularis. Hamathecium comprising few, septate, hyaline, thin-walled paraphyses, longer than asci. Asci $85-120 \times 16-22 \mu \mathrm{m}(\bar{x}=101.7 \times$ $18.5 \mu \mathrm{m}, \mathrm{n}=20$ ), 8-spored, unitunicate, cylindrical to fusiform, short pedicellate, apex rounded, with J- apical ring. Ascospores $45-55 \times 7-8 \mu \mathrm{m}(\bar{x}=48.6 \times 7.3 \mu \mathrm{m}, \mathrm{n}=20)$, uniseriate or biseriate, fusiform to ellipsoid, with long basal appendage, with 1 median and 1 basal septum, slightly constricted at the septa, smooth-walled, apical cell slightly wider than basal cell, appendage long filiform, straight or curved, with slight apical swelling, germ tubes emerging at the median septa and ascospores becoming olivaceous at germination. Asexual morph - Undetermined.

Culture characters - Colonies growing on MEA becoming $2 \mathrm{~cm}$ within 7 days, circular, flat to umbonate, margin filiform, dark green, tightly attached to the substrate, forming erect mycelium.

Material examined - ITALY, Province of Trento, Mezzana, Marilleva 900, on dead branch of Alnus incana (L.) Moench (Betulaceae), 15 August 2013, Erio Camporesi, IT 1415 (MFLU 162868 holotype; ibid BBH isotype); ex-type living cultures MFLUCC 13-0793, TBRC.

Notes - Ranulospora alnii was collected from bark of grey alder. This taxon appears to be a saprobe and several ascomata were grouped together with the necks combined by brown, fungal hyphae. The ascospore morphology is unique and we have not observed any taxon like this within Sydowiellaceae, although such appendages occur in other families (such as Ceriospora in Annulatascaceae (Campbell et al. 2013)). Phylogenetically this taxon forms a well-supported clade, which is sister to Cainiella and Alborbis (Fig. 1). 

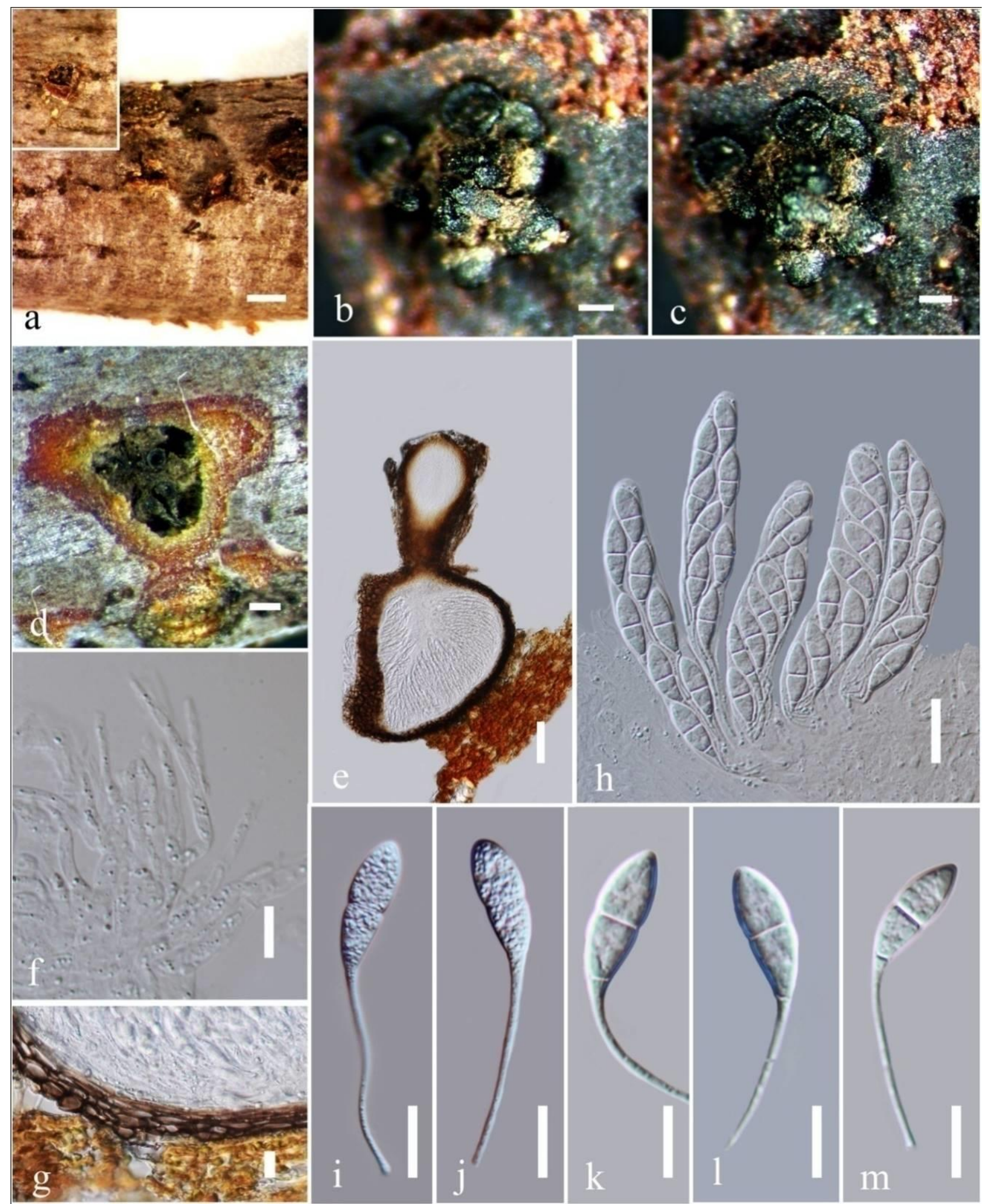

Figure 14 - Ranulospora alnii (holotype). a-d Appearance of ascomata on host substrate. e Vertical section of ascoma. f Paraphyses. g Peridium. h Asci. i-m Ascospores with basal appendage. Scale bars $-\mathrm{a}=500 \mu \mathrm{m}, \mathrm{b}-\mathrm{d}=200 \mu \mathrm{m}, \mathrm{d}, \mathrm{e}=100 \mu \mathrm{m}, \mathrm{g}, \mathrm{h}=20 \mu \mathrm{m}, \mathrm{f}, \mathrm{i}-\mathrm{m}=10 \mu \mathrm{m}$.

Rossmania Lar.N. Vassiljeva, Mycoscience 42(4): 401 (2001)

Facesoffungi number - FoF 2855

Saprobic on dead woody bark. Sexual morph - Ascomata immersed under the bark, in clusters with convergent, long, stout erumpent necks, without an ectostromatic disc, globose, coriaceous, black. Necks wide, opening through the ostiole, internally covered by hyaline periphyses, comprising 


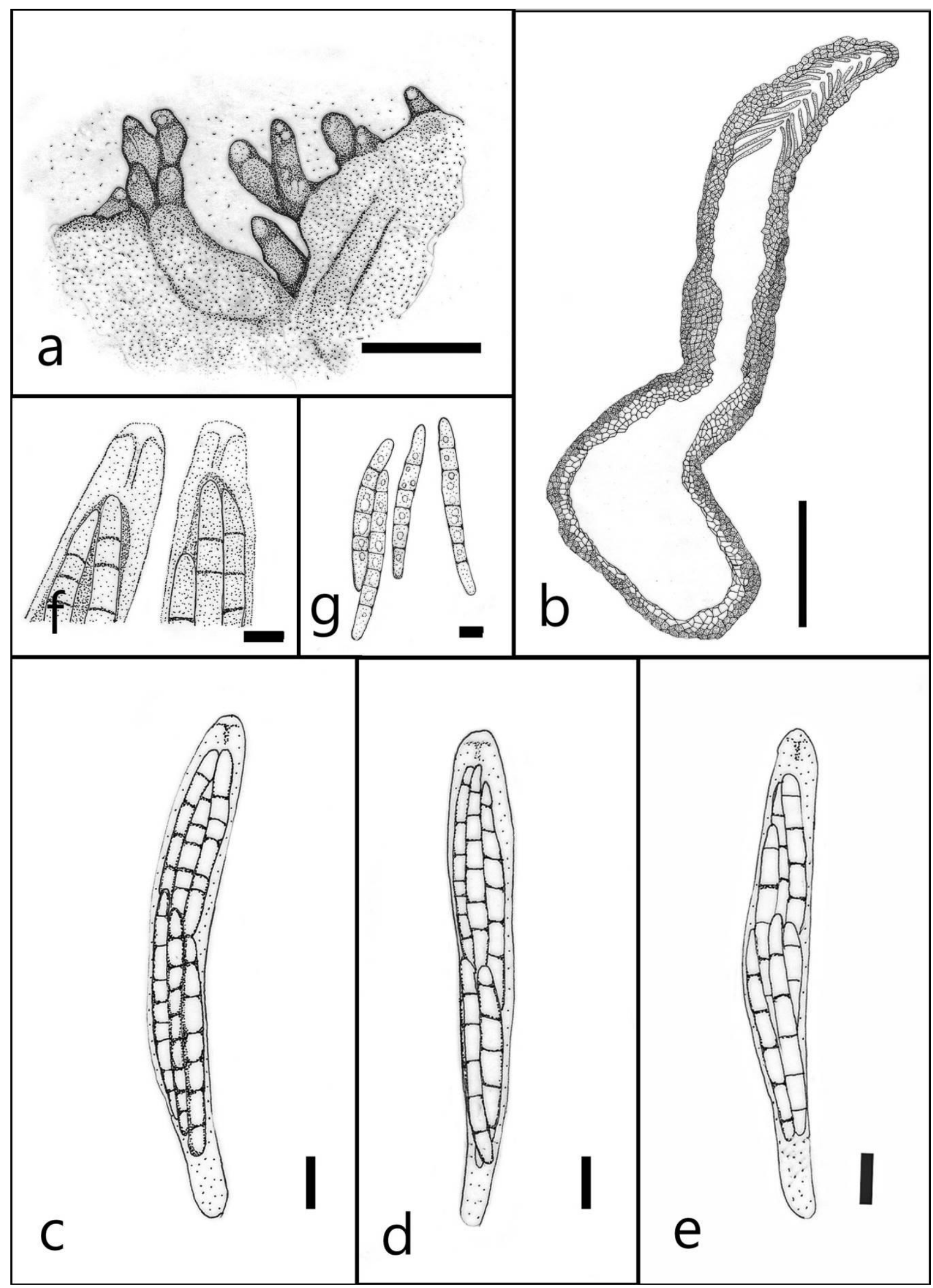

Figure 15 - Rossmania ukurunduensis (redrawn from Vasilyeva 2001). a Appearance of ascomata on substrate. $b$ Vertical section of ascoma. c-e Asci. f Apical ring. g Ascospores. Scale bars $-\mathrm{a}=1$ $\mathrm{mm}, \mathrm{b}=200 \mu \mathrm{m}, \mathrm{c}-\mathrm{e}=20 \mu \mathrm{m}, \mathrm{f}, \mathrm{g}=10 \mu \mathrm{m}$. 
brown cells of textura porrecta. Peridium thick, comprising outer, thick-walled, brown cells of textura angularis and inner thick-walled, hyaline cells of textura angularis at the base. Hamathecium aparaphysate. Asci 8-spored, unitunicate, clavate to cylindrical, short pedicellate, apex rounded, lacking an apical ring. Ascospores 4-5-seriate to fasciculate, long fusoid to cylindrical, slightly curved, 0-11-septate, hyaline to light yellow. Asexual morph - Undetermined.

Type species - Rossmania ukurunduensis Lar.N. Vassiljeva, Mycoscience 42(4): 401 (2001)

Rossmania ukurunduensis Lar.N. Vassiljeva, Mycoscience 42(4): 401 (2001)

Facesoffungi number - FoF 2856

Isotype - BPI 747566

Fig. 15

Saprobic on dead bark of Acer ukurunduense Wall. Sexual morph - Ascomata immersed under the bark, with clusters of convergent, long, stout erumpent necks, without a ectostromatic disc, globose, coriaceous, black. Necks wide, opening through the ostiole, internally covered by hyaline periphyses, comprising brown cells of textura porrecta. Peridium thick, comprising outer, thick-walled, brown cells of textura angularis and inner thick-walled, hyaline cells of textura angularis at the base. Hamathecium aparaphysate. Asci 190-230 × 24-28 $\mu \mathrm{m}, 8$-spored, unitunicate, clavate to cylindrical, short pedicellate, apex rounded, lacking an apical ring. Ascospores 76-125 × 7-9 $\mu \mathrm{m}, 4-5$-seriate to fasciculate, long fusoid to cylindrical, slightly curved, $0-7$-septate in Melzer's reagent, not constricted at the septa, hyaline, sometimes guttulate. Asexual morph - Undetermined.

Notes - Rossmania was introduced to accommodate taxa similar to Diaporthe, in its clusters of ascomata with long beaks and non-united ectostromatic disc, but differs in its septate elongate ascospores (Vasilyeva 2001). Therefore, superficially this genus is similar to Diaporthe or Phragmodiaporthe, but differs in having elongate Winterella-like ascospores. Rossmania comprises $R$. ukurunduensis and $R$. aculeata (Petch) Lar.N. Vassiljeva, the latter transferred from Phragmodiaporthe aculeata (Petch) Wehm., because it lacks true phragmospores (Vasilyeva 2001). Rossmania ukurunduensis differs from $R$. aculeata in having narrow, 5-7-septate ascospores, while $R$. aculeata has wider, 7-11-septate ascospores (Vasilyeva 2001). Rossmania ukurunduensis was collected in Russia associated with Acer ukurunduense, while $R$. aculeata was collected from Sri Lanka associated with Theaceae (Vasilyeva 2001). Vasilyeva (2001) placed Rossmania in Valsaceae. However, Rossman et al. (2007) and Lumbsch \& Huhndorf (2010) placed Rossmania in Sydowiellaceae based on its morphology. The placement of Rossmania within Sydowiellaceae has been confirmed in phylogenetic studies (Fig. 1) (Kruys \& Castlebury 2012, Maharachchikumbura et al. 2015). It differs from Winterella and Sillia by non stromatic ascoma and short cylindrical ascospores.

Sillia P. Karst., Bidr. Känn. Finl. Nat. Folk 23: 20, 159, 251 (1873)

Facesoffungi number - FoF 2857

Saprobic on dead stems of deciduous trees. Sexual morph - Stroma erumpent through cracks in bark, hemisphaerical, black on the surface, dark yellow internally, becoming rouge in $10 \%$ $\mathrm{KOH}$. Ascomata immersed in stromatic tissues, arising as several layers, globose, coriaceous, black, with cylindrical necks, protruding above the surface of the stroma. Peridium comprising thick-walled, brown cells of textura angularis. Asci 8-spored, unitunicate, narrowly clavate, short pedicellate, with a distinct small apical structure. Ascospores fasciculate, narrowly fusiform, slightly curved, hyaline, with 3-5-transverse septa, sometimes guttulate. Asexual morph Undetermined.

Type species - Sillia ferruginea (Pers.) P. Karst., Bidr. Känn. Finl. Nat. Folk 23: 159 (1873)

Sillia karstenii Senan., Camporesi \& K.D. Hyde, sp. nov.

Index Fungorum number - IF552727, Facesoffungi number - FoF 2858

Fig. 16

Etymology - in honour of late Prof Petter Adolf Karsten, an eminent mycologist who introduced the genus Sillia. 


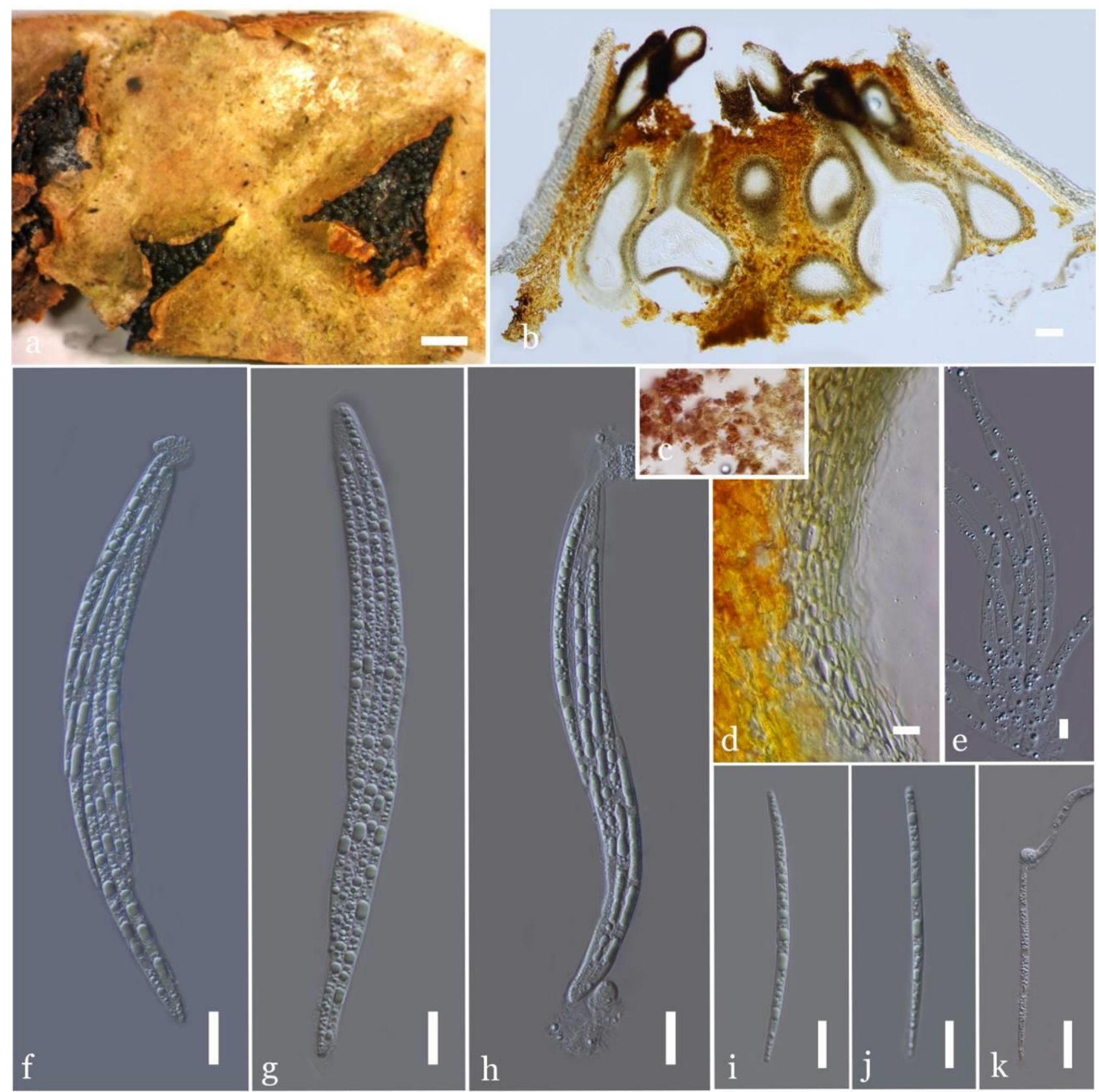

Figure 16 - Sillia karstenii (holotype). a Herbarium specimen. b Vertical section of stroma showing ascomata. c Stromatic tissues become umber in $10 \% \mathrm{KOH}$. d Peridium. e Paraphyses. f-h Asci. i-k Ascospores. Scale bars $-\mathrm{a}=1 \mathrm{~mm}, \mathrm{~b}=100 \mu \mathrm{m}, \mathrm{d}, \mathrm{f}-\mathrm{k}=20 \mu \mathrm{m}, \mathrm{e}=10 \mu \mathrm{m}$.

\section{Holotype - MFLU 16-2864}

Saprobic on dead branches of Corylus avellana L. Sexual morph - Stromata poorly developed, pseudoparenchymatous, comprising yellowish brown cells of textura angularis, turning to umber in $10 \% \mathrm{KOH}$. Ascomata $720-1200 \mu \mathrm{m}$ high, $230-265 \mu \mathrm{m}$ diameter $(\bar{x}=1032 \times 255 \mu \mathrm{m}$, $\mathrm{n}=10$ ), immersed to erumpent, gregarious, globose to subglobose, black, coriaceous, papillate, ostiolate. Neck 315-700 $\mu \mathrm{m}$ long, 50-140 $\mu \mathrm{m}$ wide $(\bar{x}=432 \times 70 \mu \mathrm{m}, \mathrm{n}=10)$, symmetrical, erumpent through host surface, internally covered by hyaline periphyses, darker than peridium cells at the base. Peridium 35-50 $\mu \mathrm{m}$ thick $(\bar{x}=47 \mu \mathrm{m}, \mathrm{n}=15)$, comprising thick-walled, brown, compressed cells of textura angularis. Hamathecium comprising thin-walled, septate, unbranched, hyaline paraphyses $6-10 \mu \mathrm{m}$ wide $(\bar{x}=8 \mu \mathrm{m}, \mathrm{n}=15)$. Asci $175-230 \times 15-18 \mu \mathrm{m}(\bar{x}=200 \times 17$ $\mu \mathrm{m}, \mathrm{n}=25), 8$-spored, unitunicate, cylindrical, straight or somewhat curved, with a short obtuse pedicel. Ascospores $110-120 \times 4.5-6 \mu \mathrm{m}(\bar{x}=114 \times 5.2 \mu \mathrm{m}, \mathrm{n}=30)$, overlapping bi-seriate, 
filiform with pointed ends, 5-6-septate when mature, guttulate, smooth-walled, ends forming a swelling when germinating. Asexual morph - Undetermined.

Material examined - ITALY, Province of Forlì-Cesena, near Bertinoro, dead branch of Corylus avellana L (Corylaceae), 30 December 2013, Erio Camporesi, IT 1611 (MFLU 16-2864, holotype, ibid $\mathrm{BBH}$, isotype).

Notes - Winter (1887) accommodated Sillia species with hyaline scolecosporous ascospores in the genus Melogramma which has brown phragmospores. Therefore, Lindau (1897) separated them again and placed Sillia in Melogrammataceae. Wehmeyer (1926) considered Sillia to be similar to Cryptospora Tul. (= Ophiovalsa), which was placed in Diaporthaceae. Ascospores of Sillia are filiform or acicular, scolecosporous and multi-septate. Ophiovalsa has unicellular, longcylindric-fusoid ascospores. However, the presence or absence of septa is not thought to be a significant character in scolecosporous spores (Tibpromma et al. 2017). Hence Sillia was accepted as closely related to Ophiovalsa in Diaporthaceae. Munk (1953, 1957) and Dennis (1960) also treated Sillia as a separate genus of Diaporthaceae. However, Barr (1978) included Sillia in family Gnomoniaceae (Tribe Endothieae). Later, Rossman et al. (2007) transferred Sillia in Sydowiellaceae based on its morphology. Several phylogenetic studies supported the taxonomic placement of Sillia in Sydowiellaceae (De Silva et al. 2009, Kruys \& Castlebury 2012). The type species Sillia ferruginea has been reported from various countries in the northern hemisphere, including Austria, Bulgaria, Canada, Czechoslovakia, Denmark, England, France, Germany, Ireland, Poland, Russia, Spain, Sweden, Ukraine and the USA (Farr \& Rossman 2006).

Sillia karstenii is the second species reported from Italy. There are six Sillia species listed in Index Fungorum (2017). Sillia theae Hara (on Camellia sinensis (L.) Kuntze in Japan), S. kamatii Tilak, S.B. Kale \& S.V.S. Kale (on dead stem of Gymnema sylvestre (Retz.) Schult. in India), S. celastrina R. Rao (on dead stems of Celastrus paniculata Willd. in Brazil) and S. biformis Rick from Brazil. S. albofusca Höhn. has fused papilla and thus is quite distinct from S. karstenii. The type species S. ferruginea (Pers.) P. Karst., S. italica de Silva, Camporesi \& K.D. Hyde and the new species $S$. karstenii were associated with Corylus species. Sillia karstenii also differs in having large ascomata with very poorly developed stromata. Phylogenetically, Sillia karstenii is relatively distinct from sequenced species with strong support and its establishment as a new species is justified.

Tenuiappendicula Senan., Camporesi \& K.D. Hyde, gen. nov.

Index Fungorum number - IF552728, Facesoffungi number - FoF 2859

Etymology - Name comprising two Latin words "tenuis" and "appendicula", referring to the slender ascospore appendages.

Saprobic on branches Alnus cordata (Loisel.) Duby. Sexual morph - Pseudostromata parenchymatous, comprising thin-walled, light brown cells of textura globosa. Ascomata aggregated, immersed, globose to subglobose, black, coriaceous, ostiolate, papillate. Papilla slanted to horizontal in substrate, curved, asymmetrical, sometimes convergent, widening towards the apex, internally covered by hyaline, filamentous periphyses. Peridium comprising an outer, thick-walled, light brown layer of very few compressed layers of cells of textura angularis and inner thin-walled, hyaline layer of compressed cells of textura angularis. Hamathecium aparaphysate. Asci 8-spored, unitunicate, fusiform to clavate, with short pedicel, apex blunt, lacking apical ring. Ascospores biseriate to overlapping biseriate, oval, 1-septate, thick-walled. Appendages at both ends, slender, long, hyaline. Asexual morph - Coelomycetous. Conidiomata acervuli, scattered, solitary, immersed, subglobose, unilocular, dark brown. Conidiomatal wall multi-layered, comprising thickwalled, brown, small cells of textura angularis. Conidiophores branched at the base, cylindrical, septate, hyaline, formed from the upper pseudoparenchyma, smooth-walled. Conidiogenous cells holoblastic, terminal, discrete, with 1-2 percurrent proliferations, indeterminate, cylindrical, simple, hyaline, smooth-walled. Conidia cylindrical, narrowing towards ends, with small spine at upper end, (4)-5-(6)-septate, hyaline, sometimes guttulate, thick-walled.

Type species - Tenuiappendicula alnicola Senan., Camporesi \& K.D. Hyde 
Tenuiappendicula alnicola Senan., Camporesi \& K.D. Hyde, sp. nov.

Index Fungorum number - IF552729, Facesoffungi number - FoF 2860

Figs 17,18

Etymology - Name after the host genus.

Holotype - MFLU 16-1265A

Saprobic on branch of Alnus cordata (Loisel.) Duby. Sexual morph - Pseudostromata parenchymatous, comprising thin-walled, light brown cells of textura globosa. Ascomata 180-200 $\times 50-80 \mu \mathrm{m}(\bar{x}=191 \times 78 \mu \mathrm{m}, \mathrm{n}=20)$, aggregated, immersed, globose to subglobose, black, coriaceous, ostiolate, papillate. Papilla $85-115 \times 30-40 \mu \mathrm{m}(\bar{x}=93 \times 36 \mu \mathrm{m}, \mathrm{n}=15)$, slanted to horizontal in the substrate, curved, asymmetrical, sometimes convergent, widening towards the apex, internally covered by hyaline, filamentous periphyses. Peridium $12-16 \mu \mathrm{m}(\bar{x}=14 \mu \mathrm{m}, \mathrm{n}=$ 15), comprising an outer layer of thick-walled, light brown, compressed cells of textura angularis and inner thin-walled, hyaline layer of compressed cells of textura angularis. Hamathecium 5-9 $\mu \mathrm{m}(\bar{x}=7.8 \mu \mathrm{m}, \mathrm{n}=15)$, comprising cellular, wide, septate, thin-walled paraphyses. Asci 105-120 $\times 25-35 \mu \mathrm{m}(\bar{x}=116 \times 33 \mu \mathrm{m}, \mathrm{n}=25), 8$-spored, unitunicate, fusiform to clavate, with short pedicel, apex blunt, lacking an apical ring. Ascospores $25-30 \times 7-10 \mu \mathrm{m}(\bar{x}=27 \times 8.8 \mu \mathrm{m}, \mathrm{n}=$ 25), biseriate to overlapping biseriate, oval, 1-septate, constricted at septum, thick-walled, appendaged. Appendages slender, long20-30 $\times 1.5-2.5 \mu \mathrm{m}(\bar{x}=27 \times 2 \mu \mathrm{m}, \mathrm{n}=20)$, one each at each end, hyaline. Asexual morph - Conidiomata 30-45 × 120-135 $\mu \mathrm{m}(\bar{x}=35 \times 130 \mu \mathrm{m}, \mathrm{n}=15)$, acervuli, scattered, solitary, immersed, subglobose, unilocular, dark brown. Conidiomatal wall 35$50 \mu \mathrm{m}(\bar{x}=37 \mu \mathrm{m}, \mathrm{n}=15)$, multi-layered, comprising thick-walled, brown, small cells of textura angularis. Conidiophores $16-21 \times 2.5-6 \mu \mathrm{m}(\bar{x}=18 \times 4.7 \mu \mathrm{m}, \mathrm{n}=15)$, branched at the base, cylindrical, septate, hyaline, formed from the upper pseudoparenchyma, smooth-walled. Conidiogenous cells $14-16 \times 4.5-5.5 \mu \mathrm{m}(\bar{x}=15 \times 4.8 \mu \mathrm{m}, \mathrm{n}=25)$, holoblastic, terminal, discrete, with 1-2 percurrent proliferations, indeterminate, cylindrical, simple, hyaline, smooth-walled. Conidia 35-45 $\times 4.5-5.5 \mu \mathrm{m}(\bar{x}=40 \times 5 \mu \mathrm{m}, \mathrm{n}=25)$, cylindrical, narrowing towards the ends, with a small spine at upper end, (4) -5-(6)-septate, hyaline, sometimes guttulate, thick-walled.

Culture characters - Colonies growing on MEA attained $1 \mathrm{~cm}$ within 7 days when incubated at $18{ }^{\circ} \mathrm{C}$, flat, circular, irregular, white to off-white, woolly, sometimes mycelium in tufts.

Material examined - ITALY, Province of Forlì-Cesena, Fiumicello di Premilcuore, on dead branch of Alnus cordata (Loisel.) Duby (Betulaceae), 24 April 2013, Erio Camporesi, IT 1199 (MFLU 16-1265A, holotype), ex-type living culture MFLUCC 16-1452; ibid BBH isotype, TBRC.

Notes - Tenuiappendicula forms a well-supported clade as a sister taxon to Chapeckia. Tenuiappendicula is characterized by 1-septate ascospores with long, thin appendages and asci without an apical ring. We observed the asexual morph of Tenuiappendicula associated with ascomata and we extracted genomic DNA from the conidiomata. LSU and ITS sequences derived from both sexual and asexual morphs are identical and hence the holomorph is illustrated.

Tortilispora Senan. \& K.D. Hyde, gen. nov.

Etymology - Name based on two Latin words "tortilis" and "spora" referring to the twisted ascospores.

Index Fungorum number - IF552802, Facesoffungi number - FoF 2863

Saprobic on dead twigs and branches. Sexual morph - Stromata forming angular, rather prominent pustules, with grey-brown to orange-brown ectostromatic discs, with 2-15 ascomata embedded in a well-developed ectostroma in a valsoid configuration. Ascomata immersed, aggregated, globose to subglobose, coriaceous, dark brown to black, ostiolate, papillate, paraphysate. Papilla black with periphyses. Peridium comprising thick-walled, dark brown to black, small cells of textura angularis. Asci 8-spored, unitunicate, cylindrical, sessile, apex rounded, with a J-, apical ring. Ascospores fasciculate, narrowly fusiform to long cylindrical, hyaline, transseptate. Asexual morph - Undetermined.

Type species - Tortilispora aurantiaca (Wehm.) Senan. \& K.D. Hyde 


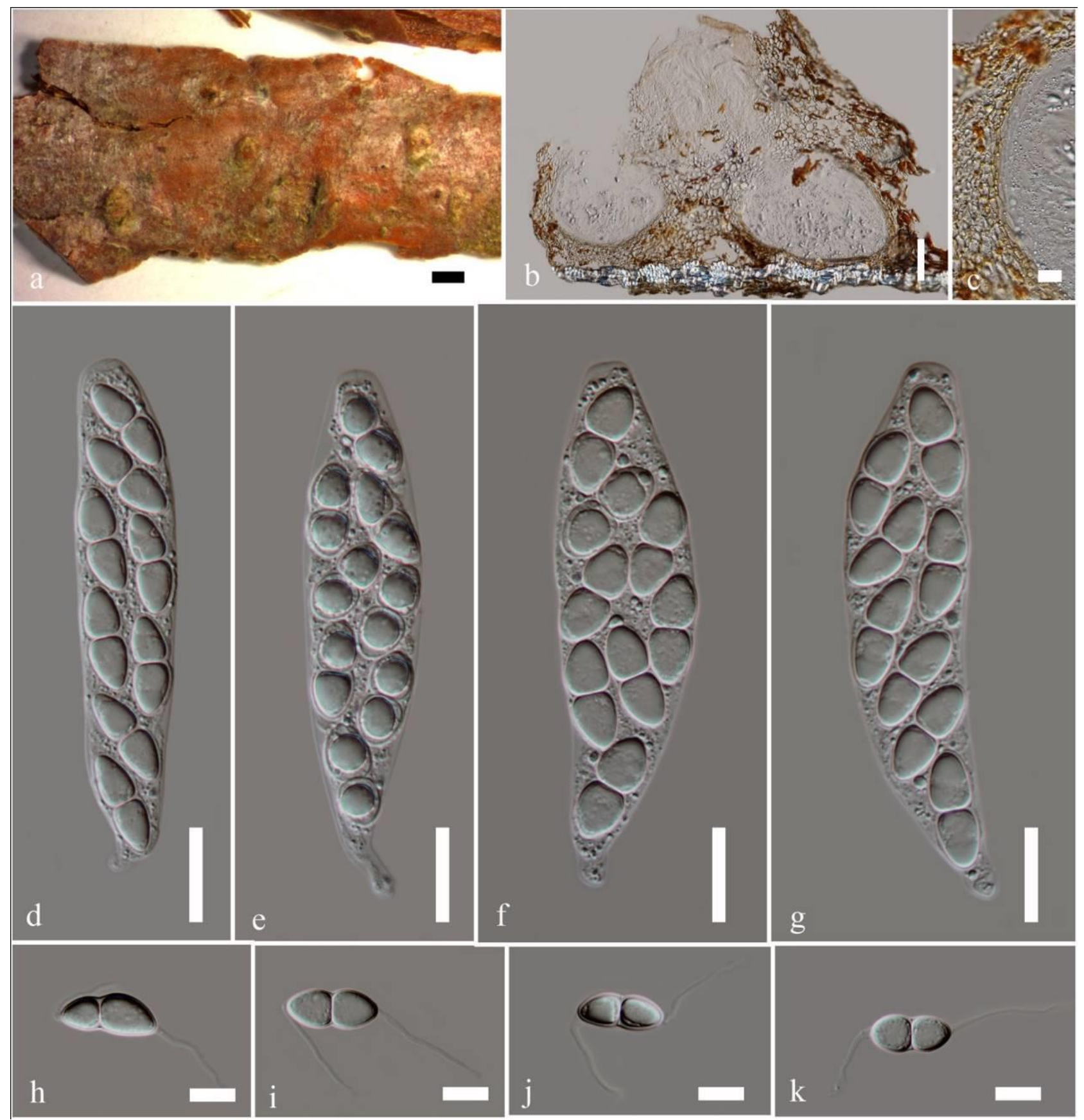

Figure 17 - Tenuiappendicula alnicola (holotype). a Herbarium specimen. b Vertical section of ascomata. c Peridium. d-g Asci. h-k Ascospores. Scale bars $-\mathrm{a}=500 \mu \mathrm{m}, \mathrm{b}=100 \mu \mathrm{m}, \mathrm{c}=20 \mu \mathrm{m}$, $\mathrm{d}-\mathrm{g}=20 \mu \mathrm{m}, \mathrm{h}-\mathrm{k}=10 \mu \mathrm{m}$.

Tortilispora aurantiaca (Wehm.) Senan. \& K.D. Hyde, comb. nov.

Index Fungorum number - IF552803, Facesoffungi number - FoF $2864 \quad$ Fig. 19

Basionym - Cryptospora aurantiaca Wehm., Canadian Journal of Research, Section C 20(12): 589 (1942)

Holotype (part) - MICH 13964

Saprobic on dead twigs and branches. Sexual morph - Stromata 0.8-3 mm diameter, forming angular, rather prominent pustules, with grey-brown to orange-brown ectostromatic discs, immersed, scattered, solitary, black, parenchymatous, with 2-15 ascomata embedded in a welldeveloped ectostroma, which consists of hyaline to subhyaline, densely compact, thin-walled hyphae, with a valsoid configuration. Ascomata 675-875 $\mu \mathrm{m}$ high, $225-300 \mu \mathrm{m}$ wide $(\bar{x}=765 \times$ $275 \mu \mathrm{m}, \mathrm{n}=25$ ), immersed, aggregated, globose to subglobose, coriaceous, dark brown to black, 
ostiolate, papillate. Papilla 375-500 $\mu \mathrm{m}$ high, 100-125 $\mu \mathrm{m}$ wide $(\bar{x}=107 \times 466 \mu \mathrm{m}, \mathrm{n}=20)$, black, comprising parallel to interwoven, thick-walled, light to dark brown hyphae, internally covered by hyaline, filamentous periphyses. Peridium $10-15 \mu \mathrm{m}$ wide $(\bar{x}=13 \mu \mathrm{m}, \mathrm{n}=25)$, comprising thick-walled, dark brown to black, small cells of textura angularis. Hamathecium comprising broad, tapering band-like, septate paraphyses. Asci 90-100 $\times 11-13 \mu \mathrm{m}(\bar{x}=93 \times 12$ $\mu \mathrm{m}, \mathrm{n}=25), 8$-spored, unitunicate, cylindrical, sessile, apex rounded at apex, with a J- apical ring, comprising two refractive bodies. Ascospores 85-95 $\times 3-4 \mu \mathrm{m}(\bar{x}=93 \times 3.5 \mu \mathrm{m}, \mathrm{n}=25)$, fasciculate, narrowly fusiform to long cylindrical, hyaline, 1-7-septate, smooth-walled. Asexual morph - Undetermined.
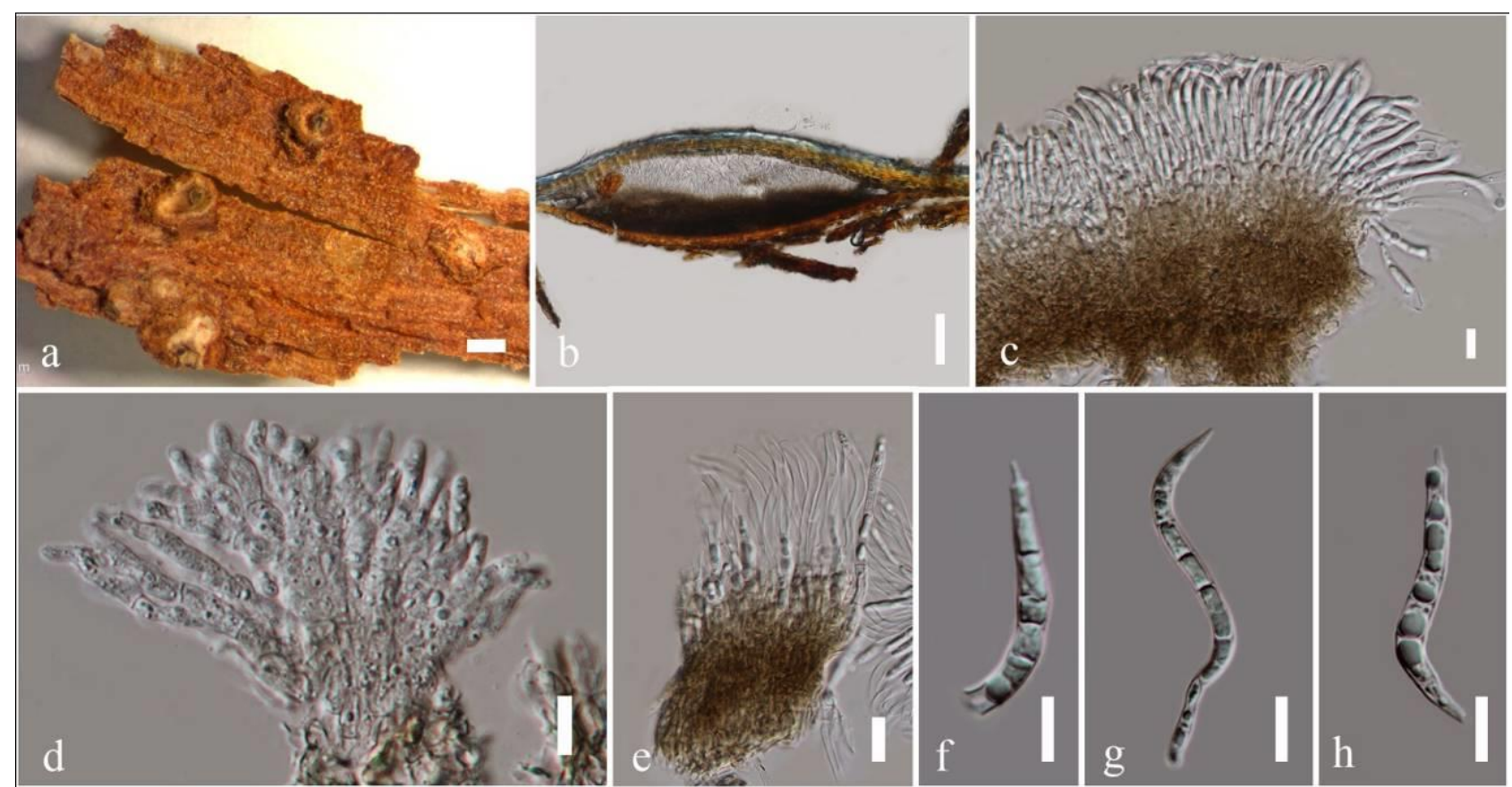

Figure 18 - Tenuiappendicula alnicola (holotype). a Herbarium specimen. b Vertical section of conidioma. c, d Conidiophores, conidiogenous cells and conidia. e Broken conidial wall attached to conidiogenous cells. f-h Conidia. Scale bars $-\mathrm{a}=500 \mu \mathrm{m}, \mathrm{b}=100 \mu \mathrm{m}, \mathrm{c}-\mathrm{e}=10 \mu \mathrm{m}, \mathrm{f}-\mathrm{h}=10 \mu \mathrm{m}$.

Material examined - CANADA, Nova Scotia, on Alnus sp. Mill. (Betulaceae), 09 July 1933, L.W. Wehmeyer, (1624), MICH (13964), holotype.

Notes - Winterella was accepted in Sydowiellaceae by Maharachchikumbura et al. (2016). There is, however, some confusion surrounding Winterella and we follow Mejia et al. (2008) and treat Ophiovalsa and Winterella as synonyms of Cryptosporella (Gnomoniaceae). Winterella albofusca, W. aurantiaca, and W. cinctula were excluded from Cryptosporella by Mejia et al. (2008) based on morphological and molecular data. LSU data (not shown) indicated that $W$. albofusca and $W$. aurantiaca belonged in the Diaporthales, although outside Gnomoniaceae. The above-mentioned species are characterized by multi-septate ascospores, and are placed in Sydowiellaceae in our phylogenetic analyses (Fig. 1). Since they do not cluster with Sillia species and are not typical of Rossmania species, genera with filiform ascospores, there is no obvious place to accommodate them. We thus introduce a new genus Tortilispora to accommodate these taxa with $T$. aurantiaca as the type species and we proposed T. albofusca (IF 552807), T. cinctula (IF552808) for other species. 


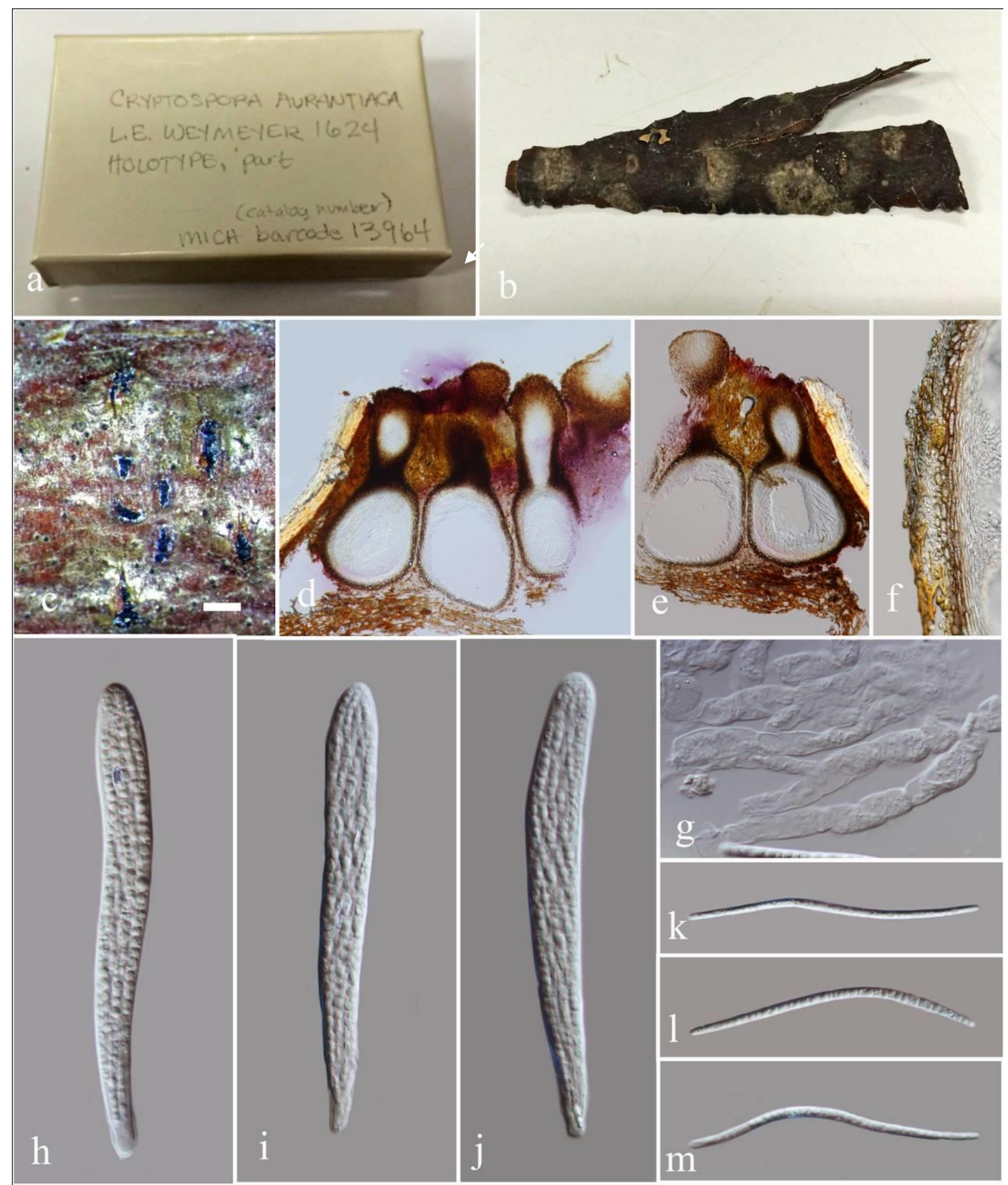

Figure 19 - Tortilispora aurantiaca (holotype). a, b Herbarium packet. d Herbarium specimen. c Ascomata on substrate. e Horizontal cross section of stromata. f, g Cross sections of ascomata. $h$ Papilla. i Paraphyses. j Peridium. k-m Asci in water. n-q Ascospores. Scale bars: c, e $=500 \mu \mathrm{m}, \mathrm{f}-\mathrm{h}$ $=100 \mu \mathrm{m}, \mathrm{i}=20 \mu \mathrm{m}, \mathrm{j}-\mathrm{q}=10 \mu \mathrm{m}$. Note - Rouge stromatic tissues in $10 \% \mathrm{KOH}$, arrowed in $\mathrm{g}$.

\section{Diaporthales genera, incertae sedis}

In this section, we deal with Uleoporthe which appears to be atypical of Sydowiellaceae.

Uleoporthe Petr., Annls mycol. 39(4/6): 279 (1941)

Facesoffungi number - FoF 2861 
Saprobic or biotrophic on recently dead plant tissues. Sexual morph - Stromata roughly circular, on the surface of leaves. Ascomata surrounding the conidiomata, deeply immersed in stromatic tissues, globose to subglobose, ostiolate, papillate, with copious paraphyses. Peridium of strongly flattened, pale brown cells of textura angularis at the base and thick-walled, hyaline cells of textura angularis in the papilla region. Asci 8-spored, unitunicate, short-pedicellate to almost sessile, apex rounded. Ascospores overlapping uniseriate to biseriate, clavate, 2-celled, hyaline. Asexual morph - Conidiomata loculate, irregular in shape, ostiolate. Peridium comprising layers of brown, thin-walled cells of textura epidermoidea. Conidiophores poorly differentiated, thin-walled, ellipsoidal. Conidiogenous cells in small clusters or short branched chains of cells, irregular or narrowly flask-shaped, tapering towards the upper region, both percurrent and sympodial proliferation, minute, slightly flared collarettes where percurrent proliferation occurs. Conidia ellipsoidal to fusiform, hyaline, aseptate (description of asexual morph from Cannon 2001). (1941)

Type species - Uleoporthe orbiculata (Syd. \& P. Syd.) Petr., Annls mycol. 39(4/6): 280

Uleoporthe orbiculata (Syd. \& P. Syd.) Petr., Annls mycol. 39(4/6): 280 (1941)

Basionym - Valsonectria orbiculata Syd. \& P. Syd., Annls mycol. 14(1/2): 86 (1916)

Facesoffungi number - FoF 2862

Fig. 20

Lectotype - W (E. Ule 3389)

Saprobic or biotrophic on recently dead plant tissues. Sexual morph - Stromata 3-7 mm diameter, roughly circular in external view, epigenous on leaves, occupying the upper epidermis and palisade layers of the leaf, erumpent, convex, initially with simple stellate splits, later becoming more complex and branched, mostly coalescing with yellow-brown host tissue at the surface, with poorly to well-developed black line around the stroma and internal eustromatic tissue with thick-walled, glassy hyaline cells of textura globulosa to textura angularis around the basal region of ascomata and dark brown cells of textura globulosa to textura angularis around the ostioles. Ascomata 435-450 $\mu \mathrm{m}$ high, $400-460 \mu \mathrm{m}$ wide $(\bar{x}=445 \times 450 \mu \mathrm{m}, \mathrm{n}=15)$, surrounding the conidiomata, deeply immersed in stromatic tissues, solitary, globose to subglobose, coriaceous, light brown, ostiolate. Papilla 190-200 $\mu \mathrm{m}$ high, 80-90 $\mu \mathrm{m}$ wide $(\bar{x}=195 \times 85 \mu \mathrm{m}, \mathrm{n}=15)$, conical, slightly eccentric, hyaline, filamentous periphysate. Peridium $15-20 \mu \mathrm{m}$ wide $(\bar{x}=19 \mu \mathrm{m}$, $\mathrm{n}=15$ ), comprising 5-6 layers of strongly flattened, pale brown cells of textura angularis at the base and thick-walled, hyaline cells of textura angularis in the ostiolar region. Hamathecium comprising copious, very thin-walled, persistent paraphyses, swollen at the base and gradually tapering towards the apex. Asci $60-80 \times 10-20 \mu \mathrm{m}(\bar{x}=65 \times 14 \mu \mathrm{m}, \mathrm{n}=25)$, 8-spored, unitunicate, narrowly clavate, short-pedicellate to almost sessile, apex rounded, sometimes slightly attenuate. Ascospores 16-20 $\times 5-6.5 \mu \mathrm{m}(\bar{x}=18 \times 5.4 \mu \mathrm{m}, \mathrm{n}=15)$, overlapping uniseriate to biseriate, clavate, upper cell ellipsoidal and the lower cylindric-ellipsoidal, with a median to slightly supramedian septum, thick-walled, hyaline, smooth-walled, each cell sometimes biguttulate. Asexual morph - Conidiomata loculate, irregularly-shaped, ostiolate. Peridium comprising 2-3 layers of brown, thin-walled cells of textura epidermoidea. Conidiophores poorly differentiated, thin-walled, ellipsoidal. Conidiogenous cells in small clusters or short branched chains of cells, irregular or narrowly flask-shaped, tapering towards the upper region, both percurrent and sympodial proliferation, minute, with slightly flared collarettes where percurrent proliferation occurs. Conidia ellipsoidal to fusiform, apex acute to obtuse, base narrowly truncate, thick-walled, hyaline, aseptate, smooth-walled.

Material examined - VENEZUELA, Miliquebe, Rio Cuquenau, in living leaves of Cybianthus fulvopulverulentus Mart. (Primulaceae), February 1910, E. Ule, 3389, W, lectotype.

Notes - The asexual morph of Uleoporthe was described by Sydow \& Sydow (1916) connected to Valsonectria orbiculata (= Uleoporthe orbiculata). However, Valsonectria is now considered a member of the Bionectriaceae and its type species, Valsonectria pulchella Speg., is clearly not conspecific with $U$. orbiculata (Hawksworth et al. 1995). Muller \& von Arx (1962) treated Uleoporthe as a synonym of Phylloporthe. However, Uleoporthe is characterized by a 


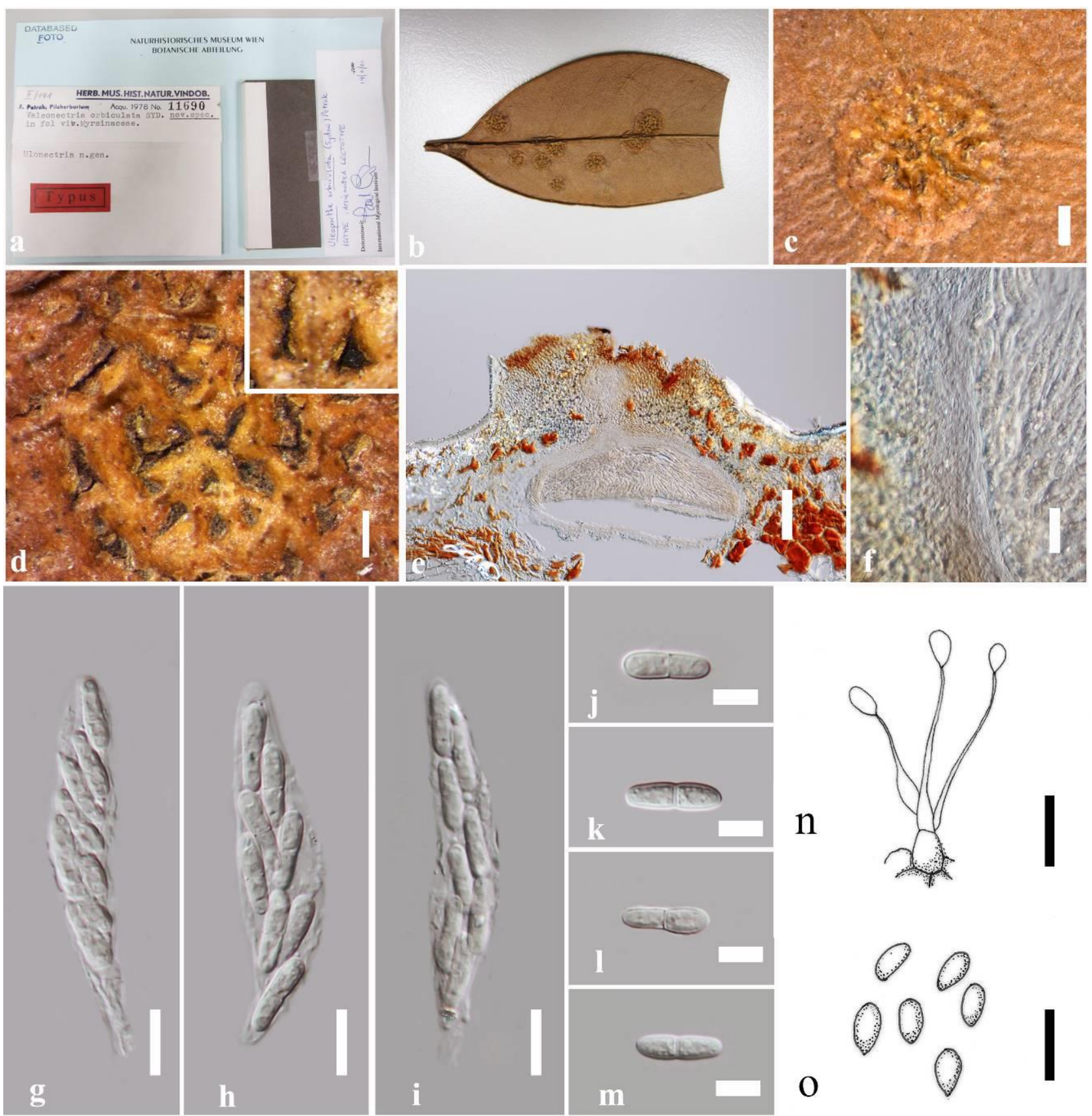

Figure 20 - Uleoporthe orbiculata (holotype). a Herbarium packet. b-d Herbarium specimen and stromata. e Cross section of ascoma. f Peridium. g-i Asci. j-m Ascospores. n Conidiophores, conidiogenous cells attached to conidia. o Conidia. Scale bars $-\mathrm{c}=1 \mathrm{~mm}, \mathrm{~d}=500 \mu \mathrm{m}, \mathrm{e}=100 \mu \mathrm{m}$, $\mathrm{f}=10 \mu \mathrm{m}, \mathrm{g}-\mathrm{i}, \mathrm{n}-\mathrm{o}=20 \mu \mathrm{m}, \mathrm{j}-\mathrm{m}=10 \mu \mathrm{m}$.

prosenchymatous stroma and hyaline, 2-celled ascospores, while Phylloporthe has a plectenchymatous to pseudoparenchymatous stroma. Phylloporthe also has strongly erumpent stromata composed mostly of fungal tissue, short pedicellate asci which are evanescent at the base, an aparaphysate centrum and periphysate ostioles (Cannon 2001). Petrak (1941) reported characters of the asexual morph of Uleoporthe as similar to Phomopsis. We could not observe the asexual morph from the lectotype. Barr (1978) did not include Uleoporthe as a synonym of Phylloporthe but placed Uleoporthe as a member of Gnomoniaceae (subfamily Stegophoroideae, tribe Stegophoreae). However, this was not accepted by Cannon (2001) based on the stromatic development and the small, acervular, spermatial asexual morph. Cannon (2001) placed Uleoporthe in Melanconidaceae, but this is problematic because the classification was developed based on exclusively temperate taxa, which may show different patterns of evolution to tropical taxa, and 
fewer asexual morph characters (Cannon 2001). Lumbsch \& Huhndorf (2010) and Maharachchikumbura et al. (2015) placed Uleoporthe in Sydowiellaceae. Sequence data for Uleoporthe is unavailable in GenBank to clarify the correct phylogenetic position. Thus, fresh collections and sequence data are needed to establish the true affinities of this taxon.

We examined the holotype of Uleoporthe orbiculata. We could not observe an apical ring in the ascus as it has a delicate wall. Morphologically, $U$. orbiculata is atypical of other taxa in Sydowiellaceae. Uleoporthe orbiculata is a leaf epiphyte forming circular, erumpent to superficial stromata, while other taxa form immersed stromata on bark. Therefore, we exclude Uleoporthe from Sydowiellaceae and place it in Diaporthales genera, incertae sedis, until sequence data is available.

\section{Acknowledgements}

K.D. Hyde thanks Kunming Institute of Botany for the award of Visiting Professor.

\section{References}

Alexopoulos CJ, Cation D. 1952 - Gnomonia fragariae in Michigan. Mycologia 44, 221-223

Ariyawansa HA, Hawksworth DL, Hyde KD, Jones EBG. et al. (2014) Epitypification and neotypification: guidelines with appropriate and inappropriate examples. Fungal Diversity 69: 57-91

Barr ME. 1959 - Northern pyrenomycetes 1. Canadian eastern arctic. Contributions Institute Botany, University Montreal 73, 1-101

Barr ME. 1978 - The Diaporthales in North America: with emphasis on Gnomonia and its segregates. Mycologia Memoirs 7, 1-232

Barr ME. 1991 - Revisions and additions to the Diaporthales. Mycotaxon 41, 287-305

Bolay A. 1972 - Contribution à la connaissance de Gnomonia comari Karsten. Étude taxonomique, phytopathologique et recherches sur sa croissance in vitro. Bericht der Schweizerischen Botanischen Gesellschaft 81, 398-482

Campbell J, Shearer CA, Crane JL, Fallah PM. 2003 - A reassessment of two freshwater ascomycetes, Ceriospora caudae-suis and Submersisphaeria aquatica. Mycologia. 95:41-53.

Cannon PF. 2001 - Rediscovery and redescription of the genus Uleoporthe (Melanconidaceae). Fungal Diversity 7, 17-25

Carbone I, Kohn LM. 1999 - A method for designing primer sets for speciation studies in filamentous ascomycetes. Mycologia 91, $553-556$

Chomnunti P, Hongsanan S, Aguirre-Hudson B, Tian Q et al. 2014 - The sooty moulds. Fungal Diversity 66, 1-36

Clements FE, Shear CL. 1931 - Genera of Fungi. 2nd ed. Wilson, New York, USA.

Currey F. 1858 - Synopsis of the fructification of the compound Sphaeriae of the Hookerian herbarium. The Transactions of the Linnean Society of London 22, 257-287

De Silva H, Castlebury LA, Green S, Stone JK. 2009 - Characterization and phylogenetic relationships of Anisogramma virgultorum and $A$. anomala in the Diaporthales (Ascomycota). Mycological Research 113, 73-81

De Silva NI, Lumyong S, Hyde KD, Bulgakov T et al. 2016 - Mycosphere Essays 9: Defining biotrophs and hemibiotrophs Mycosphere 7, 545-559

Dennis RWG. 1960 - Cryptogamic botany. In: A discussion on the biology of the southern cold temperate zone. Proceedings of the Royal Soc. Series B 152 (949), 539-540

Ellis JB, Everhart BM. 1892 - New species of fungi. Journal of Mycology 7 (2), 130-135

Ellis MA, Kuter GA, Wilson LL. 1984 - Fungi that cause cane cankers on thornless blackberry in Ohio. Plant Disease 68, 812-815

Farr DF, Bills GF, Chamuris GP, Rossman AY. 1989 - Fungi on Plants and Plant Products in the United States, APS Press, St. Paul, Minnesota 
Farr DF, Rossman AY. 2006 - Fungal Databases, Systematic Mycology and Microbiology Laboratory, ARS, USDA. URL - http://nt.ars-grin.gov/fungaldatabases/ [accessed 10-022017]

Felsenstein J. 1985 - Confidence limits on phylogenies: an approach using the bootstrap. Evolution 39(4), 783-791

Fuckel KWGL. 1863 - Fungi Rhenani exsiccati a L. Fuckel collecti. Hedwigia 2, 132-136

Glass NL, Donaldson GC 1995 - Development of primer sets designed for use with the PCR to amplify conserved genes from filamentous ascomycetes. Applied and Environmental Microbiology 61, 1323-1330

Glawe DA. 1985 - Hapalocystis berkeleyi and Macrodiplodiopsis desmazieresii in artificial culture. Mycologia 77, 880-886

Hall TA. 1999 - BioEdit: a user-friendly biological sequence alignment editor and analysis program for Windows 95/98/NT. Nucleic Acids Symposium Series 41, 95-98

Hawksworth. DL, Kirk PM, Sutton BC, Pegler DN. 1995 - Ainsworth\& Bisby's Dictionary of the Fungi. 8th edition.CAB International, Wallingford

Index Fungorum. 2017 - (http://www.indexfungorum.org/Names/Names.asp

Jaklitsch WM, Voglmayr H. 2004 - Hapalocystis occidentalis - a new species of Diaporthales from North America and a key to the species of Hapalocystis. Studies in Mycology 50, 229-234

Jayasiri SC, Hyde KD, Ariyawansa HA, Bhat J et al. 2015 - The Faces of Fungi database: Fungal names linked with morphology, molecular and human attributes. Fungal Diversity 74, 3-18

Jeewon R, Hyde KD. 2016 - Establishing species boundaries and new taxa among fungi: recommendations to resolve taxonomic ambiguities. Mycosphere 7 (11), 1669-1677

Jeewon R, Liew ECY, Hyde KD. 2002 - Phylogenetic relationships of Pestalotiopsis and allied genera inferred from ribosomal DNA sequences and morphological characters. Molecular Phylogenetics and Evolution 25, 378-392.

Jeewon R, Liew, ECY, Hyde KD. 2004 - Phylogenetic evaluation of species nomenclature of Pestalotiopsis in relation to host association. Fungal Diversity 17, 39-55.

Kirk PM, Cannon PF, Minter DW, Stalpers JA. 2008 - Dictionary of the Fungi. 10th ed. Wallingford, UK: CAB International. 1-784

Kishino H, Hasegawa M. 1989 - Evaluation of the maximum likelihood estimate of the evolutionary tree topologies from DNA sequence data. Journal of Molecular Evolution 29, 170-179

Klebahn H. 1918 - Hauptund Nebenfruchtformen der Askomuyzeten, Verlag von Gebruder Borntraeger, Leipzig

Kobayashi T. 1970 - Notes on new or little-known fungi inhabiting woody plants in Japan II. Transactions of the Mycological Society of Japan 11, 57-63

Kruys A, Castlebury LA. 2012 - Molecular phylogeny of Sydowiellaceae-resolving the position of Cainiella. Mycologia 104, 419-426

Lindau G. 1897 - Pyrenomycetineae, Laboulbeniineae. In: A. Engler \& K. Prantl (eds) Die Natürlichen Pflanzenfamilien 1(1), 321-505

Liu JK, Hyde KD, Jones EBG, Ariyawansa HA et al. 2015 - Fungal diversity notes 1-110: taxonomic and phylogenetic contributions to fungal species. Fungal Diversity 72, 1-197

Liu Y, Whelen S, Hall BD. 1999 - Phylogenetic relationships among ascomycetes: evidence from an RNA polymerase II subunit. Molecular Biology and Evolution 16, 1799-1808

Lumbsch HT, Huhndorf SM. 2009 - Outline of Ascomycota 2009 Part 1. Myconet 14, 1-42

Lumbsch HT, Huhndorf SM. 2010 - Myconet Volume 14. Part One. Outline of Ascomycota 2009. Part Two. Notes on Ascomycete Systematics. Nos. 4751-5113. Fieldiana Life Earth Sciences 1, 1-64

Lumbsch TH, Huhndorf SM. 2007 - Outline of Ascomycota: 2007. Myconet (Chicago, USA: The Field Museum, Department of Botany) 13, 1-58

Maas JL, Galleta GJ, Ellis MA. 1989 - Cane canker diseases of thornless blackberry in eastern United States. Acta Horticulturae 262, 205-208 
Maas JL. 1998 - Compendium of Strawberry Diseases, 2nd edn. APS Press, St. Paul, Minnesota. 38-39

Maharachchikumbura SS, Hyde KD, Jones EBG, McKenzie EHC et al. 2015 - Towards a natural classification and backbone tree for Sordariomycetes. Fungal Diversity 72, 199-301

Maharachchikumbura SSN, Hyde KD, Jones EBG, McKenzie EHC et al. 2016 - Families of Sordariomycetes. Fungal Diversity 79: 1-317.

Mejía LC, Castlebury LA, Rossman AY, Sogonov MV, White JF. 2008 - Phylogenetic placement and taxonomic review of the genus Cryptosporella and its synonyms Ophiovalsa and Winterella (Gnomoniaceae, Diaporthales). Mycological Research 112, 23-35

Mejía LC, Castlebury LA, Rossman AY, Sogonov MV, White JF. 2011 - A systematic account of the genus Plagiostoma (Gnomoniaceae, Diaporthales) based on morphology, host associations, and a four-gene phylogeny. Studies in Mycology 68, 211-235

Monod M. 1983 - Monographie taxonomique des Gnomoniaceae (Ascomycetes de l'ordre des Diaporthales). I. Beiheft Sydowia 9, 1-315

Morocko I, Fatehi J. 2007 - Molecular characterization of strawberry pathogen Gnomonia fragariae and its genetic relatedness to other Gnomonia species and members of Diaporthales. Mycological Research 111, 603-614

Moročko I. 2006 - Characterization of the strawberry pathogen Gnomonia fragariae, and biocontrol possibilities. Diss. (sammanfattning/summary) Uppsala : Sveriges lantbruksuniv., Acta Universitatis agriculturae Sueciae, 1652-6880

Müller E, von Arx JA. 1962 - Die Gattungen der didymosporen Pyrenomyceten. Beiträge zur Kryptogamenflora der Schweiz. 11(2), 1-922

Müller E. 1956 - Uber die neue sphaeriale Gattung Cainiella. Sydowia 10, 118-121

Müller E. 1957 - Haupt- und Nebenfruchtformen bei Guignardia Viala et Ravas. Beihefte zur Sydowia 1, 210-224

Munk A. 1953 - The system of the Pyrenomycetes. A contribution to a natural classification of the group Sphaeriales sensu Lindau. Dansk Botanisk Arkives 15, 1-163

Munk A. 1957 - Danish Pyrenomycetes. A preliminary flora. Dansk Botanisk Arkives 17, 1-491

MycoBank. 2017 - http://www.mycobank.org/

Nordskog B, Stensvand A, Heiberg N. 2003 - Fungi Occurring on Aerial Constituents of Cultivated Blackberry (Rubus fruticosus L.) in Norway. Acta Agriculturae Scandinavica, Section B, Soil \& Plant Science. 53, 21-28

Nylander JAA. 2004 - MrModeltest v2. Program Distributed by the Author. Evolutionary Biology Centre, Uppsala University

Page RDM 1996 - TREEVIEW: An application to display phylogenetic trees on personal computers. Computer Applications in the Biosciences 12, 357-358

Petrak F. 1941 - Mykologische Notizen XIV (nos 931-1000). Annales Mycologici 39, 251-349

Phillips AJL, Alves A, Pennycook SR, Johnston PR et al. 2008 - Resolving the phylogenetic and taxonomic status of dark-spored teleomorph genera in the Botryosphaeriaceae. Persoonia 21, 29-55

Phookamsak R, Norphanphoun C, Tanaka K. et al. 2015 - Towards a natural classification of Astrosphaeriella-like species; introducing Astrosphaeriellaceae and Pseudoastrosphaeriellaceae fam. nov. and Astrosphaeriellopsis, gen. nov. Fungal Diversity 74-143

Raciborski M. 1900 - Parasitische Algen und Pilze Java's. H. Teil, 1-46

Reid J, Booth C. 1987 - Winterella, the correct name for Cryptospora and Ophiovalsa. Canadian Journal of Botany 65, 1320-1342

Ronquist F, Teslenko M, van der Mark P, Ayres DL et al. 2012 - MrBayes 3.2: efficient Bayesian phylogenetic inference and model choice across a large model space. Systematic Biology 61, $539-42$ 
Rossman AY, Castlebury LA, Samuels GJ. 2006 - Phylogeny and biodiversity of the Hypocreales and Diaporthales. Eighth International Mycological Congress, 21-25, August 2006, Cairns. Congress Handbook \& Abstracts, Book 2

Rossman AY, Farr DF, Castlebury LA. 2007 - A review of the phylogeny and biology of the Diaporthales. Mycoscience 48, 135-144

Saccardo PA. 1883 - Sylloge Fungorum, vol. 2. Saccardo, Pavia, Italy.

Schröter J. 1897 - Pilze. In: Cohn F (ed), Kryptogamen-Flora von Schlesien 3.2. Kern, Breslau, Germany.

Silvestro D, Michalak I. 2012 - raxmlGUI: a graphical front-end for RaxML Organisms Diversity \& Evolution 12, 335-337

Sogonov MV, Castlebury LA, Rossman AY, Mejía LC, White JF. 2008 - Leaf-inhabiting genera of the Gnomoniaceae, Diaporthales. Studies in Mycology 62, 1-79

Stamatakis A, Hoover P, Rougemont J. 2008 - A rapid bootstrap algorithm for the raxml web servers. Systematic Biology 57, 758-771

Swofford DL. 2003 -. PAUP*. Phylogenetic Analysis Using Parsimony (*and Other Methods). Version 4.Sinauer Associates, Sunderland, Massachusetts.

Sydow H, Sydow P. 1916 - Weitere Diagnosen neuer Philippinischer Pilze. Annales Mycologici $14,362-364$

Sydow H. 1936 - Novae fungorum species - XXIV. Annales Mycologici 34, 411-422

Tibpromma S, Karunarathna SC, Hyde KD, Camporesi E et al. 2017 - Fungal diversity notes 491601: taxonomic and phylogenetic contributions to fungal taxa. Fungal Diversity (in prep)

Tulasne ELR, Tulasne C. 1863 - Selecta Fungorum Carpologia 2: i-xix, 319 pp., 34 tabs. Paris; Imperial. Typograph.

Van Adriechem MCY, Bosher JE. 1958 - Leaf blotch and petiole blight of strawberry caused by Gnomonia fructicola. Plant Disease Reporter 42, 772-775

Vasilyeva L. 2001 - Pyrenomycetes of the Russian Far East-Additions and corrections. 1. Rossmania ukurunduensis gen. et sp. nov. Mycoscience. 42, 399-401

Vasilyeva LN. 1987 - Pirenomitsety i Lokuloaskomitsety severa Dalbnego Vostoka: 1-255

Vasilyeva LN. 1998 - Nizshie Rasteniya, Griby i Mokhoobraznye Dalnego Vostoka Rossii. 4, 1419

Vilgalys R, Hester M. 1990 - Rapid genetic identification and mapping of enzymatically amplified ribosomal DNA from several Cryptococcus species. Journal of Bacteriology 172, 4239-4246

Voglmayr H, Jaklitsch WM. 2014 - Stilbosporaceae resurrected: generic reclassification and speciation. Persoonia 33, 61-82

Wehmeyer LE. 1926 - A biologic and phylogenetic study of the stromatic Sphaeriales. American Journal of Botany 13, 575-645

Wehmeyer LE. 1933 - The British species of the genus Diaporthe Nits. and its segregates. Transactions of the British Mycological Society 17, 237-295

Wehmeyer LE. 1941 - A revision of Melanconis, Pseudovalsa, Prosthecium and Titania. University of Michigan Studies Scientific Series 14, 1-161

White TJ, Bruns T, Lee S, Taylor J. 1990 - Amplification and direct sequencing of fungal ribosomal RNA genes for phylogenetics. In: PCR Protocols: a guide to methods and applications. (Innis MA, Gelfand DH, Sninsky JJ, White TJ, eds). Academic Press, New York, USA, 315-322

Winter G. 1887 - Pilze, Ascomyceten. In: Rabenhorst's Kryptogamen-Flora von Deutschland, Oesterreich und der Schweiz [i-vi] 1(2), 1-928 